\title{
Population and Decay of Superdeformed Nuclei probed by Discrete and Quasi-continuum $\gamma$-ray Spectroscopy
}

\author{
A. Lopez-Martens ${ }^{1}$, T. Lauritsen ${ }^{2}$, S. Leoni ${ }^{3,4}$, T. Døssing ${ }^{5}$, T.L. Khoo ${ }^{2}$ and S. Siem ${ }^{6}$ \\ ${ }^{1}$ CSNSM, IN2P3-CNRS, and Université Paris Sud, Bat. 104-108, F-91405 Orsay, France \\ 2 Argonne National Laboratory, Argonne, Illinois 60439, USA \\ ${ }^{3}$ INFN, Sezione di Milano, I-20133 Milano, Italy \\ ${ }^{4}$ Dipartimento di Fisica, University of Milano, I-20122 Milano, Italy \\ 5 The Niels Bohr Institute, University of Copenhagen, D-2100 Copenhagen, Denmark \\ ${ }^{6}$ Department of Physics, University of Oslo, N-0316 Oslo, Norway
}

February 17, 2016

\begin{abstract}
Nuclear superdeformation at high spin was discovered a little over 30 years ago. Since then, a large body of data has been collected on the subject and many new and interesting phenomena have been discovered. In particular, the way superdeformed states are populated and depopulated offers a unique laboratory to study rotational motion as a function of excitation energy and the evolution of nuclear structure over a large interval in energy and spin. This article focusses on the experimental techniques and methods developed to study the quasicontinuous spectra of gamma rays emitted by rapidly rotating superdeformed nuclei and presents the results regarding rotational damping, the transition from ordered to chaotic motion and quantum tunnelling in a complex environment.
\end{abstract}

\section{Introduction}

\section{$1.1 \quad$ General Properties}

The nucleus, with a finite number of nucleons, is a mesoscopic object. The mesoscopic nature is reflected in the fact that its energy can be given in terms of a superposition of a macroscopic liquid-drop term and a microscopic quantal shell-correction term. In a major advance in nuclear theory, Strutinsky [1] developed the method to compute the shell-correction energy. The shell energy has a profound impact on nuclear behaviour and leads to a number of striking consequences. One is the increased binding (by up to $7 \mathrm{MeV}$ ) in the heaviest elements, with atomic number up to 118, resulting in the creation of a fission barrier, where classically none would exist at all. The existence of these elements is possible only because of the extra stabilization from the shell-correction energy. Hence, the production and discovery of the heaviest elements with $\mathrm{Z}$ up to 118 [2,3,4] constitute some of the most exciting achievements in nuclear science.

Another striking example is the subject of this review, where the shell correction creates a secondary minimum, or false vacuum, in the potential energy surface of the nucleus, at a deformation larger than the one corresponding to the lowest minimum (true vacuum), as illustrated in Fig. 1. At the superdeformed (SD) minimum, corresponding to an elongated shape, the ratio of the long to short axes 


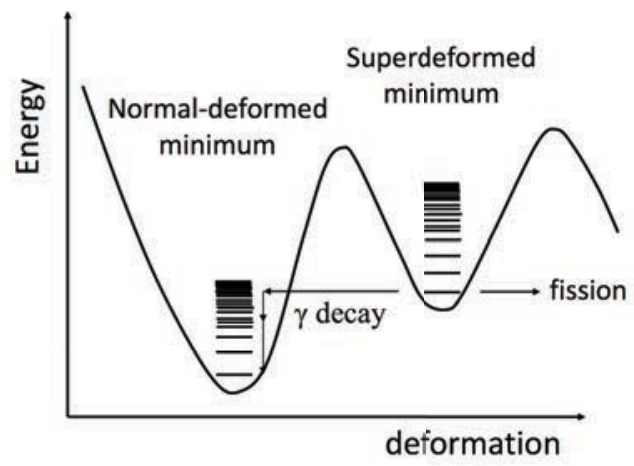

Figure 1: Sketch of potential energy vs. deformation, showing two minima created by the shell-correction energy, the lower-energy one with normal deformation, the excited one with elongated or SD shape.

is 1.5 - 2. The first manifestation of a secondary minimum was the occurrence of fission isomers [5] (reviewed in Ref. [6]). A later manifestation was the existence of SD bands, first discovered in ${ }^{152} \mathrm{Dy}$ by Twin et al [7], which exhibit impressive series of almost equi-spaced (picket-fence) $\gamma$ transitions, characteristic of a rotational spectrum; see reviews in, for example, Refs. [8, 9]. Examples of $\gamma$-ray spectra from SD bands in ${ }^{152} \mathrm{Dy}$ and ${ }^{194} \mathrm{Hg}$ are shown in Fig. 2.
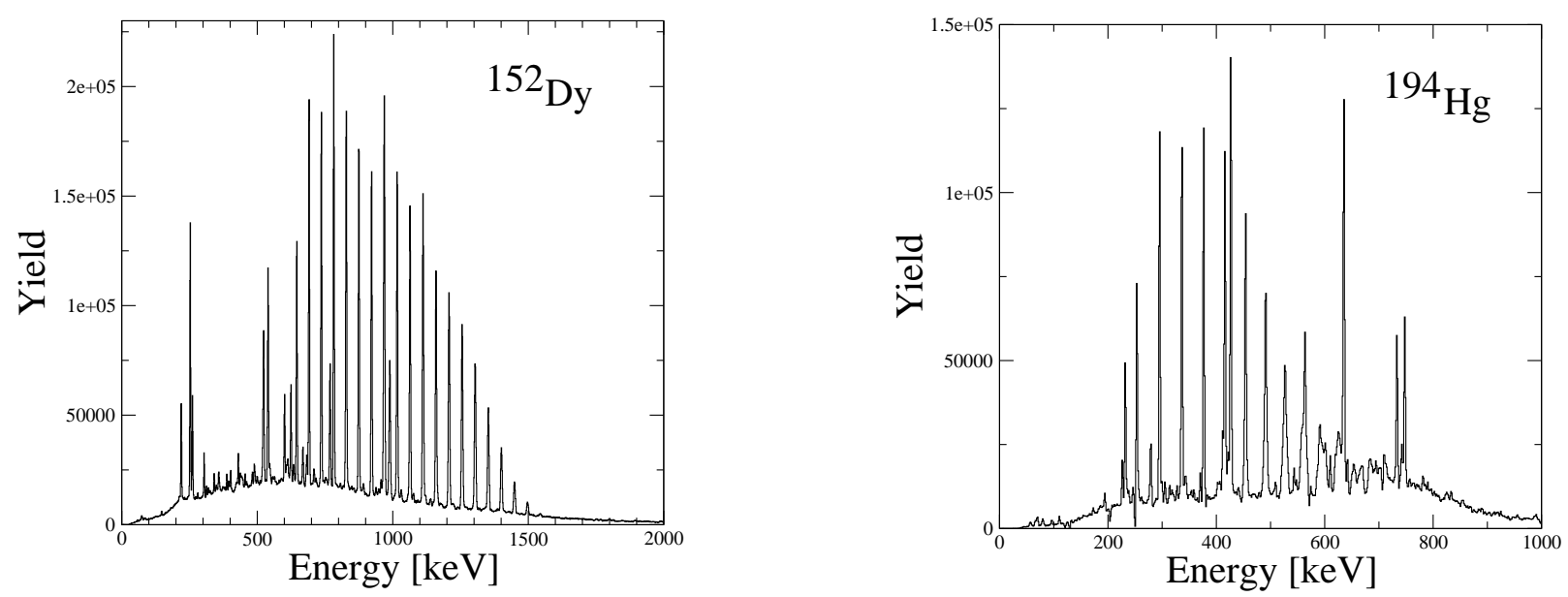

Figure 2: Spectra from ${ }^{152} \mathrm{Dy}$ and ${ }^{194} \mathrm{Hg}$, obtained by requiring a coincidence with a pair of SD lines.

Superdeformation is to date, one of the most extreme phenomena in nuclear physics in terms of deformation and electromagnetic emission. It made it to the top 10 unexpected physics discoveries (as reported by D. Kleppner in Physics Today, 1991) along with high-T superconductivity, supernovae 1987A and buckyballs. The occurrence of superdeformation owes it origin to large shell gaps. In a harmonic oscillator potential, energy gaps occur when the ratio of the long to short axes is a rational ratio [10], e.g. 2/1, corresponding to closed classical orbits. The existence of a spin-orbit interaction in nuclei modifies the pure harmonic oscillator potential, but the occurrence of shell gaps at large deformation persists. When the proton and neutron numbers are both favorable for the occurrence of shell gaps, superdeformation is found. This leads to SD nuclei, which congregate in local regions in the chart of nuclides, with mass numbers around 40, 60, 80, 130, 150, 190 and 240 (see Fig. 3, from Ref. [11]).

Although the lowest state in the SD well is at high energy (several $\mathrm{MeV}$ ) above the ground state of the nucleus, it is isolated from levels outside the well. Hence, it may be considered as a cold ground state 


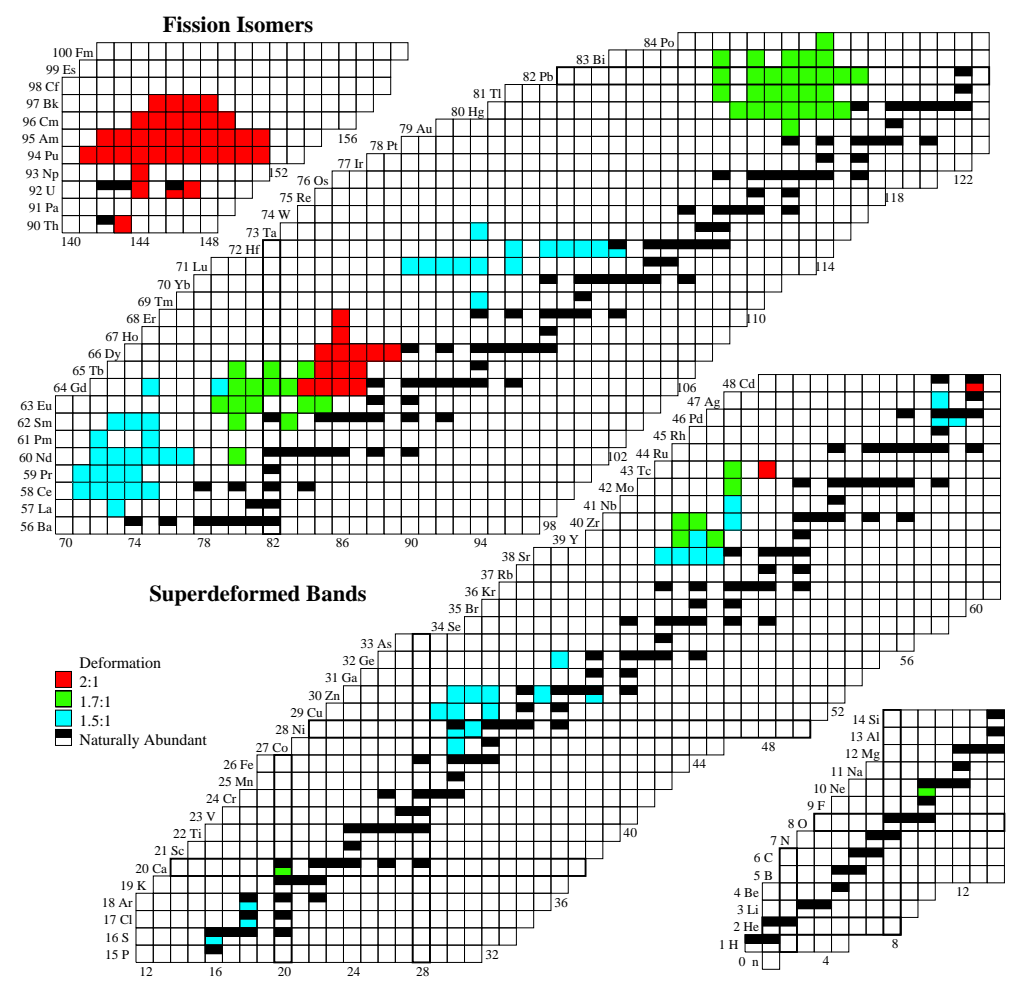

Figure 3: Chart of nuclides, showing local regions of superdeformation in the A $\sim 40,80,130,150,190$ and 240 regions; The chart has been adapted and updated by M. Birch and B. Singh [11] (McMaster Univ., Apr 30, 2015) from the original in Table of Superdeformed Nuclear Bands and Fission Isomers by Singh et al [12].

in that well, with pair correlations, and exhibits well-established rotational bands. The low-lying excited states, formed by breaking pairs or by vibrational modes, can also give rise to similar striking rotational bands. At higher excitation energies, where the level density increases, the rotational transitions are no longer discrete but form a broad quasicontinuum (QC) spectrum of E2 character. In addition, interband transitions may compete and, at high enough excitation energy, may even decay to normally-deformed (ND) states outside the well.

This review concentrates on the evolution from cold to excited SD states, the properties of the excited states, how the SD levels are populated, how they deexcite to ND states via a tunnelling process, and the experimental and theoretical methods for extracting the information.

The bulk of the research effort on superdeformation has focussed on identifying rotational bands, i.e. cold cascades. This subject has been covered by previous reviews [8, 9], so only highlights will be summarized in the following section.

\subsection{Highlights of superdeformed rotational bands}

The large body of data have been used to extract - from the spacings between transitions - the dynamical moment of inertia $\Im^{(2)}$, to infer the microscopic structure of the bands from comparisons with theoretical values. About 360 SD bands [13] have been identified. However, in all but a handful of cases, the connections of the excited SD bands to the ND yrast states have proven elusive, so that the excitation energies, spins and parities are not established. (An yrast state is defined as the lowest-lying level at each spin.) 
Lifetime measurements, on the other hand, have been feasible, showing unambiguously that the bands have large deformation (see, e.g., Ref. [14]). In the mass 190 region, many SD bands have identical quadrupole moments [14] and most have very close $\Im^{(2)}(\omega)$ vs. $\omega$ curves [9], which slope up with $\omega$. (Here, $\omega$ is the rotational frequency, given by $\mathrm{E}_{\gamma} / 2$.) The upsloping $\Im^{(2)}(\omega)$ curves have been attributed to alignment of high-j single-particle orbitals, the presence of pair correlations within the SD well [9] and the decrease of pairing with spin.

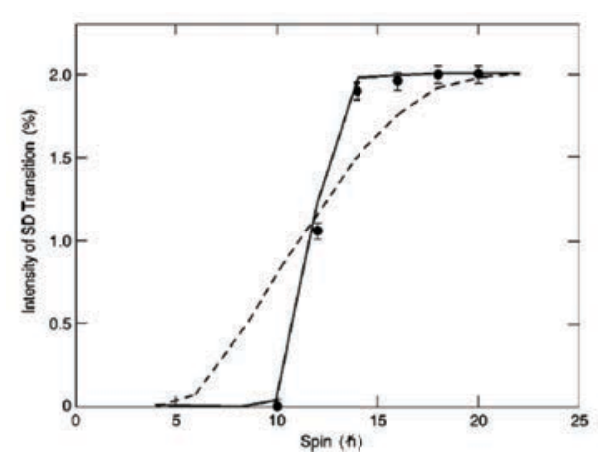

Figure 4: SD population vs. spin in ${ }^{194} \mathrm{Hg}$ [15]. Note the sudden drop at spin 10, from the rapid decay out of the SD to ND states. The continuous and dashed lines represent calculated intensities with assumptions of a SD well depth which decreases with spin or remains constant.

One surprising discovery has been the phenomenon of identical bands, where many SD bands in neighbouring nuclei have either equal (within $1 \mathrm{keV}$ ) transition energies or equal $\Im^{(2)}$ (see, e.g., Ref. [16]). There was early excitement that this phenomenon might have a "heroic" explanation based on a fundamental physics origin. However, it now seems more likely that it was an accidental (but still interesting) effect $[17,16]$, as in heavy shell-stabilized nuclei [18], arising from nearly constant quadrupole deformation in a localized mass region and, hence, close moments of inertia.

There are three intriguing SD bands (in ${ }^{149} \mathrm{Gd},{ }^{148} \mathrm{Eu}$ and ${ }^{148} \mathrm{Gd}[19,20]$ ) with identical $\Im^{(2)}$, where every other transition is slightly displaced by $\approx 500 \mathrm{eV}$ with respect to the intermediate transition when plotted against a smooth reference. An interesting speculation [21] invoked a small symmetry breaking of $\mathrm{Y}_{44}$ type around the symmetry axis to explain the $\Delta I=4$ bifurcation from tunnelling between the four energy minima. However, the inclusion of a hexadecapole deformation in the nuclear Hamiltonian cannot account for the magnitude of the experimental $\Delta I=4$ staggering pattern [22] and so the underlying reason for the observed oscillations is still not understood.

In some nuclei of the Lu-Hf region, strongly deformed triaxial bands have been observed. A few $\left({ }^{163,165,167} \mathrm{Lu}[23,24,25]\right)$ have been characterized as wobbling modes [26], which can only arise with triaxial shapes, thereby establishing the existence of stable trixiality in nuclei.

A fascinating feature of almost all SD bands is the sudden drop in intraband transition intensity at low spin (Fig. 4), after a string of up to 20 consecutive transitions. Why does the SD band disappear and why so precipitously? After a long sequence of transitions within the false vacuum, there must be a sudden decay to the true vacuum. How can it be so sudden when there is such a drastic change of shape and wave function? What happens to the $\gamma$ flux? Physicists have long sought the missing SD flux, which presented challenges to both experiment and theory. Another striking aspect occurs in SD bands in nuclei around ${ }^{56} \mathrm{Ni}$, where near the bottom of the bands, both $\gamma$ and particle emission (proton and $\alpha$ ) occurs - see, e.g., [27, 28]. How does the major rearrangement of shape and wave function occur with these "simple" emissions? These questions will be addressed in the review. 


\subsection{Properties of excited superdeformed states}

Discrete line spectroscopy is a well-established tool for investigating nuclear structure along and near the yrast line. To study the structure of higher-lying states, from which the $\gamma$ spectral density is so dense as to form a nearly-smooth spectrum, one is required to examine the entire $\gamma$-ray spectrum, not only discrete lines, but also unresolved transitions.

The total spectrum allows us to address many fascinating topics, which would otherwise not be accessible. These topics include: the feeding and decay mechanisms of SD bands; tunnelling from the SD to the ND well; the role of quantum numbers and chaos in the tunnelling; the order-to-chaos transition; the structure of excited SD bands, e.g. the evolution with spin and energy of K conservation, ordered rotational motion, rotational spreading width, dispersion in alignments, deformation/lifetime; and the coupling between hot states in the ND and SD wells. ( $\mathrm{K}$ is the projection of the total angular momentum on the symmetry axis.)

It has been possible to follow the $\gamma$ cascade from the initial hot compound nucleus to the cold yrast line and down to the ground state with the following arsenal of tools. One can isolate the $\gamma$ spectrum associated with each stage of the cascade, based on characteristic features such as the spectral shape, energy distribution, angular distribution (multipolarity), and Doppler shift (lifetime). The entry distribution in spin I and excitation energy $\mathrm{E}^{*}$ of the starting points of the cascade can be measured through $\gamma$ calorimetry, or the average entry point from the components of the total $\gamma$ spectrum. The feeding cascade ends when it enters the yrast line. With the initial and end points of the cascade specified, the region in $\left(\mathrm{I}, \mathrm{E}^{*}\right)$ probed is known. (It is also possible to inspect cascades which end at higher spin by setting coincidence gates on high-spin transitions, although this feature has not yet been exploited.) Multi-dimensional correlations, mostly $\gamma-\gamma$, provide information on the spread $\Gamma_{\text {rot }}$ of $\mathrm{E} 2$ strengths. The magnitude of this spread, called rotational damping width, can be inferred from the widths of rotational correlation patterns (called "ridges") parallel to the diagonal - see, e.g. Ref. [29]. The width of the ridges also provides information on the dispersion of alignments, $\mathrm{K}$ conservation and the separation of the moments of inertia of excited rotational bands. Fluctuation analyses of one- and two-dimensional spectra reveal the fragmentation of the cascade, i.e. the number of pathways taken. Finally, an interpretation of the physics is tested and confirmed by Monte Carlo simulations of the $\gamma$ flow (with application of the same trigger conditions as in the experiment) to check if the simulations reproduce the features enumerated above. These methods are covered in detail later in this review.

\subsection{Compound nucleus formation and $\gamma$ decay}

SD bands are populated in fusion-evaporation reactions, where a compound nucleus is formed by the fusion of a heavy-ion projectile and a target nucleus (see Fig. 5). The hot compound nucleus evaporates several light particles (typically 4 or 5 neutrons), giving rise to a nucleus at high excitation energy and spin, populating a region referred to as the entry distribution. From here, in a normal case, the nucleus cools monotonically by emission of $\gamma$ rays, until the cascade reaches the yrast line and then ends at the ground state.

The evolution of shapes, the modes of generating angular momentum and the corresponding location in the $\beta-\gamma$ plane are schematically illustrated in Fig. 5. (Here $\beta$ and $\gamma$ are shape parameters characterizing the quadrupole and triaxial shapes.)

There are additional stages of electromagnetic radiation that are more interesting when a SD well exists than when it is absent. The $\gamma$ cascade that flows through a SD well has four stages, as illustrated in Fig. 6: (a) cooling, feeding and trapping into the SD well; (b) intraband transitions within SD bands; (c) decay from SD to ND states; and (d) cooling to the ND ground state. In stage (a), hot compound nuclear states cool via $\gamma$ emission. This stage involves the coupling of hot SD and ND states, which includes tunnelling between hot states on either side of the barrier. A small fraction (typically a few 

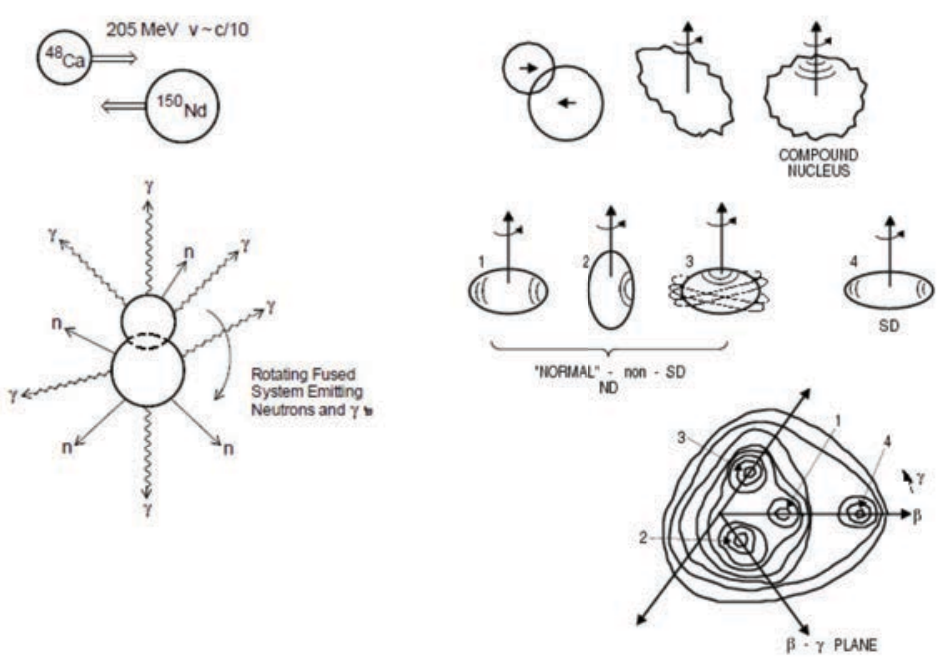

Figure 5: Left side: schematic depiction of the formation of a compound nucleus, and its decay by neutron and $\gamma$ emission. Right side. Top: evolution of nuclear shapes. Bottom: schematic depiction of shapes and the modes of generating angular momentum, with the corresponding location in the $\beta-\gamma$ plane.

$\%$ ) of the cascades become trapped in the SD minimum, leading to stage (b), where the elongated shape is established as the nucleus cools into or near the bottom of the false vacuum (with typically $1 \%$ intensity). Although the SD minimum is at high excitation energy, no further cooling occurs; isolated by the barrier, the nucleus is now at zero temperature, characterised by good quantum numbers and symmetries, and the decays are governed by selection rules. (Temperature is defined as the mean excitation energy per nucleon). Cold cascades along the SD yrast line ensue, with highly-collective E2 transitions $(\approx 2000$ Weisskopf Units) connecting members of rotational bands. This phase gives rise to the spectacular spectrum of nearly equal spacings (see Fig. 2), characteristic of collective, ordered rotation.

As can be seen in Fig. 6, with decreasing spin, a very unusual phenomenon follows: instead of the normal cooling, the excitation energy of the trapped SD state above the ND yrast line rises! As a consequence, it becomes embedded in a sea of ND states with increasing level density. As the spacing between ND states decreases, the SD state comes unavoidably close to a ND level, resulting in a small coupling and a sudden tunnelling from the cold SD state to the hot ND states - stage (c). Finally, in stage (d) the hot ND states cool to the the yrast line and then to ground state.

Several interesting aspects of the tunnelling process are worth noting. The initial SD state is a cold ordered state, characterised by good quantum numbers, whereas the final hot states are (probably) not. At high excitation energy, say near the neutron separation energy, hot ND states are described as chaotic states [30,31], where quantum numbers are largely lost - apart from energy, spin and parity. The hot ND states (with 4-5 MeV heat energies) to which the SD states tunnel appear to be chaotic (although it still remains to be established whether full chaos is reached).

By analogy with a classical system, where chaos implies no constants of motion, in a quantum system chaos implies a loss of quantum numbers. As B. Mottelson states [32], "the issue of order and chaos in nuclear spectra can be seen as the problem of characterizing the manner in which the additional quantum numbers (isospin, quasiparticle quantum number, rotational and vibrational quantum numbers) are destroyed, and the consequences this has for the different properties of the system".

The usual case of cooling from a compound nucleus to the cold ground state represents a single, gradual chaos-to-order cycle. When the flow goes through the SD well, there is a unique phenomenon 
with two cycles: chaos-to-order-to-chaos-to-order, with the tunnelling from the SD to the ND wells representing a sharp order-to-chaos transition.

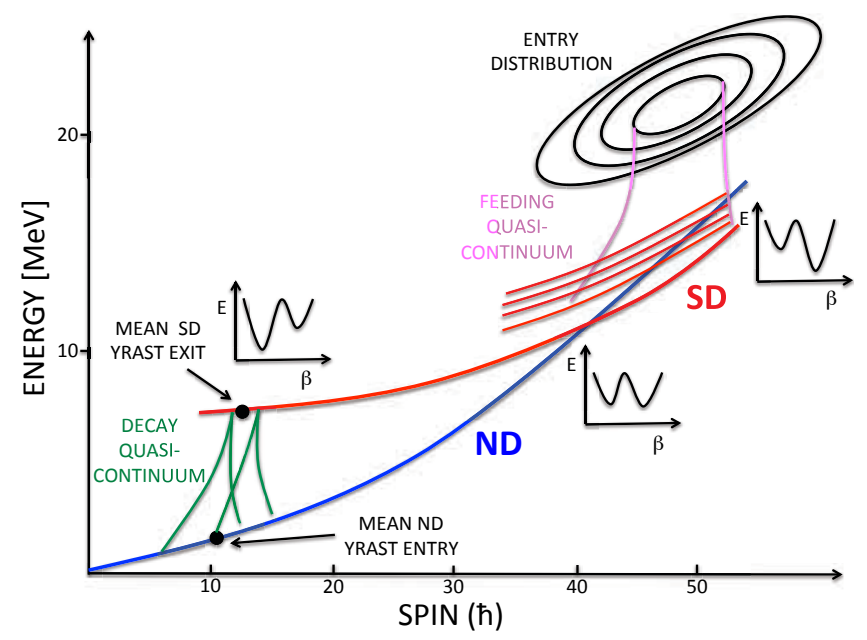

Figure 6: Schematic depiction of ND and SD yrast lines, with sketches of the ND and SD minima at different spins. Also shown are the entry distribution, from which feeding via $\gamma$ decay of SD states originates, and the decay out of SD states.

We now briefly describe the different components of the $\gamma$ spectrum, starting with the statistical $\gamma$ decay, which will be a recurring topic. The decay from a hot compound state is schematically illustrated in Fig. 7. A hot state de-excites by primary (i.e. first-step) transitions to the near-yrast states, resulting in sharp $\gamma$ lines at high energy. The predominant decay however is to higher-lying states, which are favoured by rapidly increasing level density, so that the lines overlap, form structures, and then smear into an unresolved spectrum, which is referred to as the QC. Finally, transitions between near-yrast states appear as sharp lines at low energy. These are indeed the features observed in the $\gamma$ spectrum following thermal neutron capture [33]; see Fig. 7 from Refs. [33, 34]. The statistical $\gamma$ rays provide the main cooling mechanism for the nucleus.

When the nucleus cools sufficiently in the SD well to establish an elongated shape, the still-excited, rapidly rotating object emits $\mathrm{E} 2 \gamma$ rays, yielding a broad electric quadrupole (E2) QC distribution. Finally, there is cooling to the yrast line in a change from "statistical" to "ordered" regimes, followed finally by sharp peaks in the yrast decay.

\subsection{Tunnelling in a complex quantum system}

Superdeformation in nuclei - as well as fission - provides a laboratory for studying tunnelling in a complex quantum system, a subject of much interest [35]. Many interesting questions arise. What is the role of conserved quantum numbers, such as K, in tunnelling? How do the good quantum numbers dissolve in the tunnelling process? Each specific combination of quantum numbers will be characterised by its own potential energy surface and, hence, its specific barrier characteristics. One might also ask if the formalism developed for chaos-assisted tunnelling [36, 37] could be usefully applied to the nuclear problem, or whether tunnelling from SD states can provide an interesting example of chaos- assisted tunnelling. What is clear is that, in the heavier nuclei, the chaotic (or almost chaotic) nature of the excited ND states play a critical role in facilitating tunnelling out the SD minimum and the subsequent $\gamma$ emission. In fact, Aaberg [38] has cited this feature as an example of chaos-assisted tunnelling. Finally, 

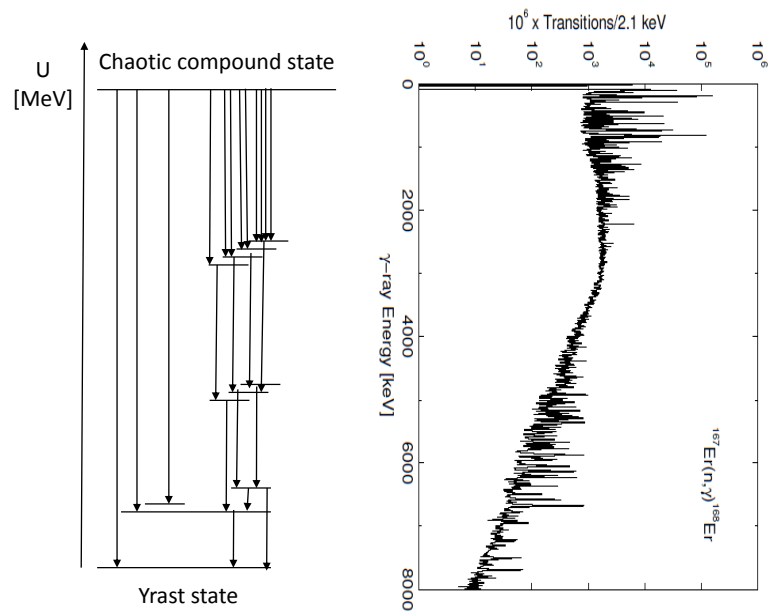

Figure 7: Decay of a compound nuclear state via $\gamma$ rays (left) and the resulting statistical spectrum - from the ${ }^{167} \operatorname{Er}(\mathrm{n}, \gamma)$ reaction (right) - showing a broad quasicontinuum component, plus sharp lines from high-energy primary decays and low-energy near-yrast transitions.

one should be aware that the tunnelling could occur in a multidimensional space, as the nuclear shape is characterised by at least two parameters, $\beta$ and $\gamma$.

This review is organised as follows: First the experimental techniques used to select and extract the spectra of interest will be presented, with special emphasis on QC spectra. This section is then followed by theoretical considerations regarding the feeding and decay-out stages of a superdeformed nucleus' life. The last section of this review is dedicated to the experimental results concerning the properties of excited states SD and ND states and their coupling, as revealed by the analysis of the feeding and decay out cascades.

\section{Experimental techniques}

The observation of superdeformation at high spin was made possible thanks to the development of arrays of escape-suppressed Ge detectors. In 1986, SD ridges were observed in ${ }^{152}$ Dy in a $\gamma-\gamma$ coincident matrix by B. Nyako and collaborators [29] using 6 Compton-suppressed Ge detectors (TESSA2). A few years later, the discrete SD band was observed by P. Twin et al. [7] with TESSA3 (12 Ge detectors). Some of the first attempts to extract and understand the QC of $\gamma$ rays, that feeds the yrast or near yrast states, were done with small $\gamma$-ray detector arrays such as: the BGO array at Argonne, the TESSA array at Daresbury [39], the GASP array at Legnaro [40], the HERA array [41] at LBNL, the $8 \pi$ array in Canada [42], the OSIRIS array [43] in Germany, Nordball [44] in Denmark and the Chateau de Cristal [45] in France. These arrays are sometimes referred to as "second generation" arrays and a more complete list can be found in [46, 47]. In fact, some of the first extractions of QC spectra with Ge detectors for ND ${ }^{152}$ Dy were done with the BGO array $[48,49]$. The important unfolding technique was developed at the BGO array as well $[50,51]$. "Unfolding" refers to techniques used to remove $\gamma$ rays that Compton scatter rather than being fully absorbed in the Ge detector and also missed the Compton veto shields. The technique of unfolding allows us to extract the true QC preceding discrete $\gamma$ rays. To extract good QC spectra from SD nuclei required the bigger and better "third generation" arrays such as EUROBALL (EB) [52] or GAMMASPHERE (GS) [53]. The latest generation of arrays, which have no Compton-suppression shields and therefore can have a much larger density of active Ge detector 
material, use tracking algorithms to track each $\gamma$ ray and recover the full energy rather than reject the Compton scattered $\gamma$ rays $[54,55]$. These "forth generation" tracking arrays, GRETINA [56] and AGATA [57], have much potential, but have yet to prove their effectiveness when it comes to high-spin and QC spectroscopy [58]. The techniques for the latter tracking arrays to properly "unfold" spectra have yet to be developed because of the difficulty of measuring the proper response function. Thus, so far, most discrete and QC SD spectra have been extracted using non-tracking, Compton suppressed, high purity Ge detector arrays. In the following we shall describe the experimental tools at hand and throughout use ${ }^{152} \mathrm{Dy}$ and ${ }^{194} \mathrm{Hg} \gamma$ rays spectra to illustrate the techniques: extraction of QC spectra in one and two dimensions, entry distribution measurement and fluctuation analysis.

\subsection{Extraction of quasi-continuum spectra}

To extract the spectra of $\gamma$ rays from nuclei that are SD usually requires placing double gates (i.e., requiring specific energy coincidences) on the discrete SD lines of the nucleus. Looking at the cartoon in Fig. 6, it is clear that a spectrum with gates on SD lines in a nucleus will contain a number of distinguishable components. First there is a feeding QC of $\gamma$ rays as the nucleus decays and gets trapped in the SD well. Once it is trapped there, the nucleus will emit the beautiful picket fence of $\gamma$ rays so prominently seen in Figs. 2 and 8. Next follows another component of the QC, when the nucleus decays into the ND well and then, finally, there will be a number of discrete $\gamma$ rays emitted while the nucleus decays to the ground state in the ND well. This section is devoted to the techniques used to extract the feeding and decay QC components.

\subsubsection{The components of a gated $\gamma-$ ray spectrum}

Fig. 2 show the spectra from ${ }^{152} \mathrm{Dy}$ and ${ }^{194} \mathrm{Hg}$ after double gates on SD lines in the nuclei have been applied. The spectra are background subtracted, using the methods described in [59], but have otherwise not been processed. There are a number of components in these spectra, as discussed in section 1.4, which we need to understand and ultimately extract. The feeding QC of $\gamma$ rays contains the statistical E1 transitions governed by eq. 30 in Sec. 4.1.1, then follows the emission of collective E2 $\gamma$ rays [60]. The final decay into states on or near the SD yrast line is by M1/E2 transition, characterized by large negative $A_{2}$ angular distribution factors (see eq. 1). As mentioned above, then follows the characteristics discrete SD $\gamma$ rays. The decay out QC in principle has the same statistical E1 transitions, collective E2 transitions and M1/E2 transitions, but the proportions of the components may be different from the feeding QC, principally because the decay happens at much lower spins. For ${ }^{194} \mathrm{Hg}$ the decay-out QC is largely a statistical spectrum; but for ${ }^{152} \mathrm{Dy}$, model calculations indicate that there is a significant E2 component as well [59, 61]. Finally, a number of discrete $\gamma$ rays are emitted in the ND well as the nucleus cools down and reaches the ground state. In the following sections we will show how these many components of the spectra are identified and extracted.

\subsubsection{One dimensional quasi-continuum spectra extraction and decomposition}

To extract clean $\gamma$-ray spectra for the investigation of the QC requires great care and a large number of procedures. For the case of ${ }^{152}$ Dy, these procedures are discussed in details in Ref. [59]. Fig 8 shows the spectra for ${ }^{152} \mathrm{Dy}$ and ${ }^{194} \mathrm{Hg}$ after background subtraction, unfolding and correction for the interaction of more than one photon in the detectors. The spectra have been normalized to the number of $\gamma$-ray cascades. They can be compared to the original (i.e., unprocessed) spectra in Fig. 2. Indeed, the removal of the Compton scattered $\gamma$ rays discussed in Ref. [59] reveals the true components of the spectra.

To find the QC, the discrete lines must be removed. This is sometimes done using a procedure that relies on a known level scheme [62] or using an automatic procedure described in [59] which removes 

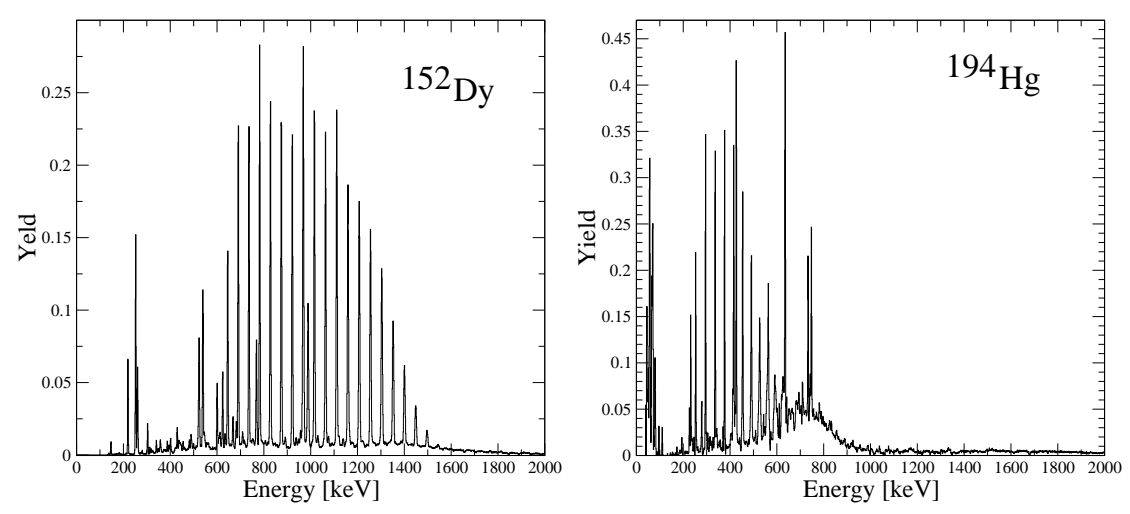

Figure 8: Sum of double SD gated spectra of ${ }^{152} \mathrm{Dy}$ and ${ }^{194} \mathrm{Hg}$ after some of the processing described in the text has been applied. Both the SD lines and ND lines in coincidence with the yrast SD bands are present. Under the discrete lines are the true feeding and decay out QC of $\gamma$ rays in coincidence with the SD bands.

all lines whether known or not. The resulting QC spectra, one for each polar angle, is then typically contracted (4-32 keV/ch) for further processing. At this point the angle-sorted spectra can be fitted with the function

$$
W(\theta)=A_{0}+A_{2} P_{2}(\cos \theta)+A_{4} P_{4}(\cos \theta)
$$

where $P_{2}$ and $P_{4}$ are Legendre polynomials of order 2 and 4 , at each energy channel in order to extract the true intensity $A_{0} \mathrm{QC}$ spectrum as well as the $A_{2}$ spectrum which contains information about the multipolarity of the $\mathrm{QC} \gamma$ rays. The $A_{0} \mathrm{QC}$ spectrum is composed of three principal components: the statistical QC, the E2 quadrupole $\mathrm{QC}$ and the dipole $\mathrm{M} 1 / \mathrm{E} 2 \gamma$ rays from the decay of the nuclei into the ND or SD states at or near the yrast lines. For the simpler case of a ND QC spectrum (see Fig. 9), the components are extracted as described in the following. First, the statistical spectrum is fitted to the high-energy part of the QC spectrum with this function [63, 64, 65, 51, 62]

$$
f\left(E_{\gamma}\right)=C E_{\gamma}^{N} e^{-\frac{E_{\gamma}}{T}}
$$

Here $E_{\gamma}$ is the $\gamma$-ray energy and $C, T$ and $N$ are fitting parameters. $N$ is typically about 3 , reflecting the dipole nature of the transitions. The statistical component can be seen for the ND case in Fig.9. After correction for the angular distribution (eq. 1) and relativistic aberration, this component is then subtracted from the QC spectrum at each polar angle. As a result, only the E2 quadrupole, and M1/E2 components remain. These components are further decomposed using the angular distribution analysis carried out using eq. 1, proportionally splitting the $\mathrm{QC} \gamma$ rays according to the $A_{2}$ between the positive E2 limit and the negative M1/E2 limit (see Fig. 3 in Ref [59]).

Extracting the QC of $\gamma$ rays in coincidence with discrete $\gamma$ rays emitted in the $\mathrm{SD}$ well follows the same principles as above, but the extra QC from the decay out from the SD well makes the analysis more of a challenge. Fig. 10 (left) shows the SD QC spectrum decomposed for ${ }^{152}$ Dy Ref. [59] and Fig. 10 (right) shows the SD QC spectrum decomposed for ${ }^{194} \mathrm{Hg}$ Ref. [60].

For the case of ${ }^{194} \mathrm{Hg}$ it is possible to distinguish the decay out from the feeding QC. The excess strength above the statistical spectrum beyond $1 \mathrm{MeV}$ corresponds to the decay-out QC. In contrast, for ${ }^{152} \mathrm{Dy}$, the decay out and feeding QC spectra are merged together so much that one cannot easily experimentally distinguish one from the other. Thus, the decay out spectrum in ${ }^{152}$ Dy is extracted through a model simulation of both the feeding and decay of the SD band [59]. The decay out spectrum is mostly composed of E2 $\gamma$ rays for ${ }^{152} \mathrm{Dy}$, while it is made up of statistical E1 $\gamma$ rays for ${ }^{194} \mathrm{Hg}$. 

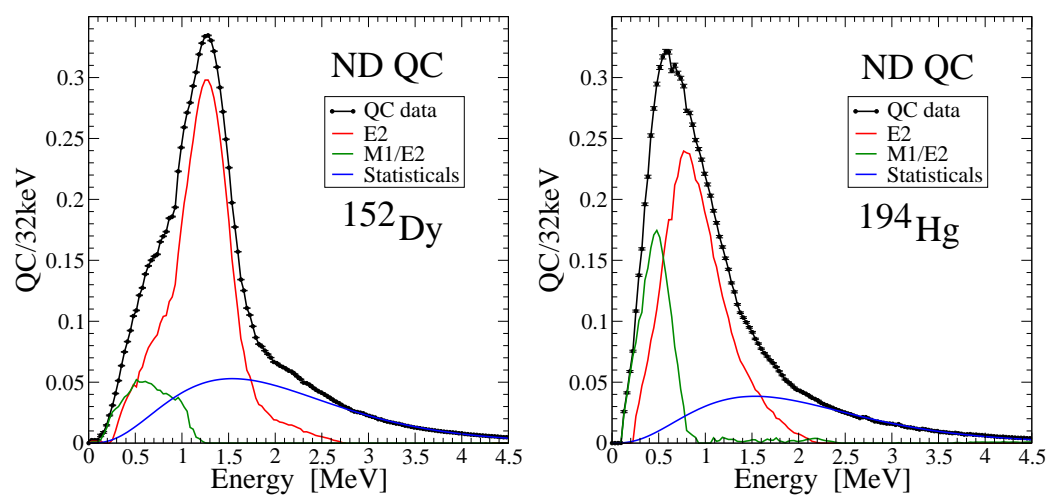

Figure 9: The QC from ${ }^{152} \mathrm{Dy}$ and ${ }^{194} \mathrm{Hg}$ when double gates are placed on ND lines [59, 15]. In both cases the three components of the feeding QC (the statisticals, the E2 $\gamma$ rays and the M1/E2 components) are shown extracted as described in section 2.1.2.

\subsubsection{The components of a $\gamma-\gamma$ matrix}

The extraction of the 1D QC spectra as described above does not reveal correlations. To extract those, one must look at the $\mathrm{QC}$ in higher dimensions, in $\gamma-\gamma$ matrices or cubes. In a $\gamma-\gamma$ matrix, there are coincidences between all the components of the total spectrum discussed in Sec. 2.1.1: ND and SD discrete lines and the feeding and decay out QC. The coincidence between the feeding E2 component of the QC will in general form ridges parallel the diagonal of the $\gamma-\gamma$ matrix, as can clearly be seen in Fig. 11. Because the E2 QC comes from many bands, with slightly different moments of inertia, the coincidences form lines parallel to the diagonal rather than discrete points. The width of these ridges (an example of these is shown in Fig. 12) along the cross-diagonal contains the information about the properties of the rotational motion of the SD nucleus at finite temperature (see Secs. 3.6 and 4.1.3).
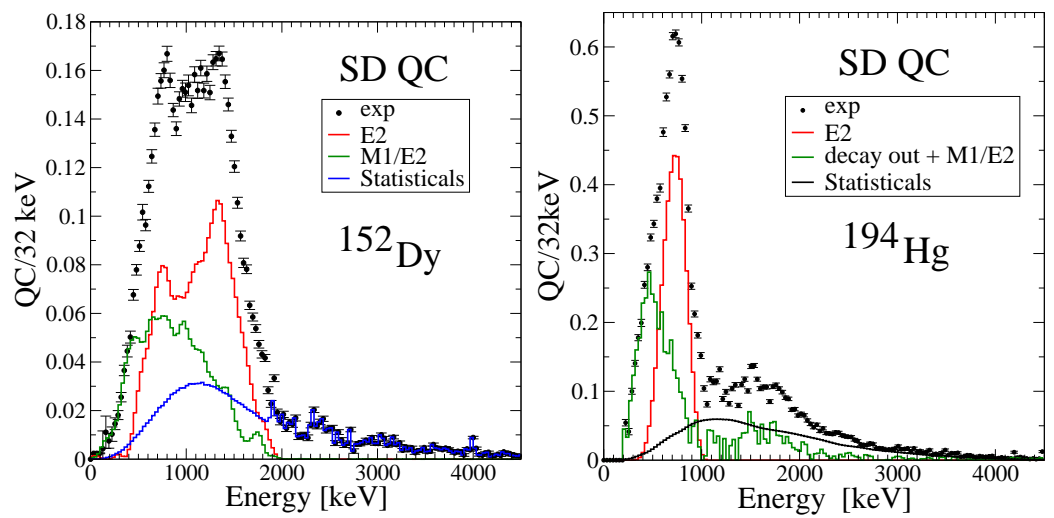

Figure 10: The SD QC decomposition for ${ }^{152} \mathrm{Dy}$ and ${ }^{194} \mathrm{Hg}$ (re-analyzed data from [59, 60]).

In addition to ridges, because the $\mathrm{E} 2 \mathrm{QC} \gamma$ rays are not in coincidence with themselves, a valley is formed along the diagonal, which rotational damping can partially fill in. As mentioned in the introduction, rotational damping corresponds to the spread in E2 transition strengths (see Sec. 3 and Sec. 4). 

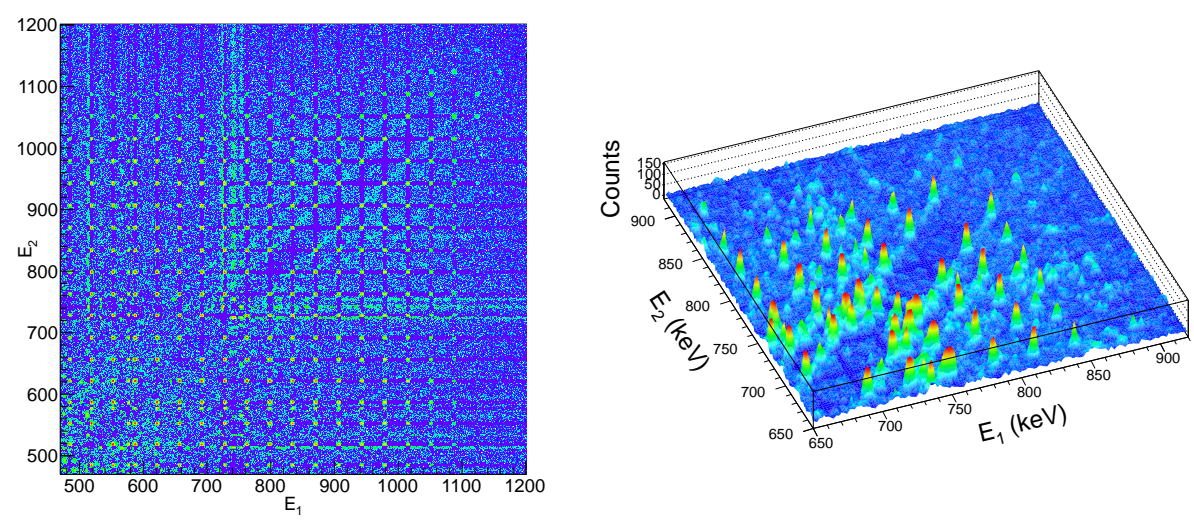

Figure 11: Examples of $\gamma-\gamma$ matrices after double gates have been placed on clean SD lines in ${ }^{152}$ Dy (left in 2D) and ${ }^{194} \mathrm{Hg}$ (right in 3D). The very regular structure of strong peaks are from discrete SD line coincidences, the more irregular coincidences are between SD and ND discrete lines or ND-ND lines. Near the diagonal, in between the SD-SD coincidences, the SD ridges are clearly visible.

\subsubsection{Two dimensional ridge extraction}

To get clean enough $\gamma-\gamma$ matrices to work with, it is usually necessary to place double gates of clean $\mathrm{SD}$ or ND lines. Thus, very high multiplicity and high statistics data is required. Just like in the 1D case, the $\gamma-\gamma$ matrix is background subtracted. This is usually done with the "COR procedure" [66], which uses as a background an uncorrelated matrix, named Uncor, generated from a projection of the original matrix:

$$
\begin{array}{r}
\operatorname{Cor}(x, y)=\operatorname{Raw}(x, y)-C \times U \operatorname{Uncor}(x, y) \\
\operatorname{Uncor}(x, y) \quad=\frac{\sum_{x} \operatorname{Raw}(x, y) \sum_{y} \operatorname{Raw}(x, y)}{\sum_{x y} \operatorname{Raw}(x, y)}
\end{array}
$$

Some groups subtract typically $70 \%$ of the counts in the matrices $(\mathrm{C}=0.7)[67]$, while others subtract so that the resulting matrix had no net counts [59, 60]. Examples of the SD ridges in ${ }^{152} \mathrm{Dy}$ and ${ }^{194} \mathrm{Hg}$ are shown in Fig. 12. Like 1D spectra, the $\gamma-\gamma$ matrices are unfolded so that only the real QC and peaks are present in the matrices. These $\gamma-\gamma$ matrices are produced so as to reveal the correlations of the E2 QC $\gamma$ rays. This is usually accomplished by projecting a portion of the matrix onto the cross-diagonal, revealing the ridges. However, the discrete peaks must first be removed. There are three approaches to this. If the level scheme is known well enough one can subtract the peaks in the $\gamma-\gamma$ matrices using the calculated peak coincidences [68, 69]. Another approach, that does not rely on having a complete level scheme, is proposed in Ref. [59]. Alternatively, one can simply directly extract the ridge in between the discrete peaks.

\subsubsection{Higher Dimensional Rotational planes}

If three-dimensional (3D) $\gamma-\gamma-\gamma$ cubes are constructed, it is possible to study the so called tilted rotational planes [71, 69], namely matrices with the triple coincidence requirement

$$
E_{\gamma 1}-E_{\gamma 3}=N \times\left(E_{\gamma 3}-E_{\gamma 2}\right) \pm \delta / 2
$$

where $\mathrm{N}$ selects the order of the plane and $\delta$ gives the thickness of the plane (typically of the order of 10-20 keV) [69], allowing for some irregularities in the rotational energy sequence. In these spectra 

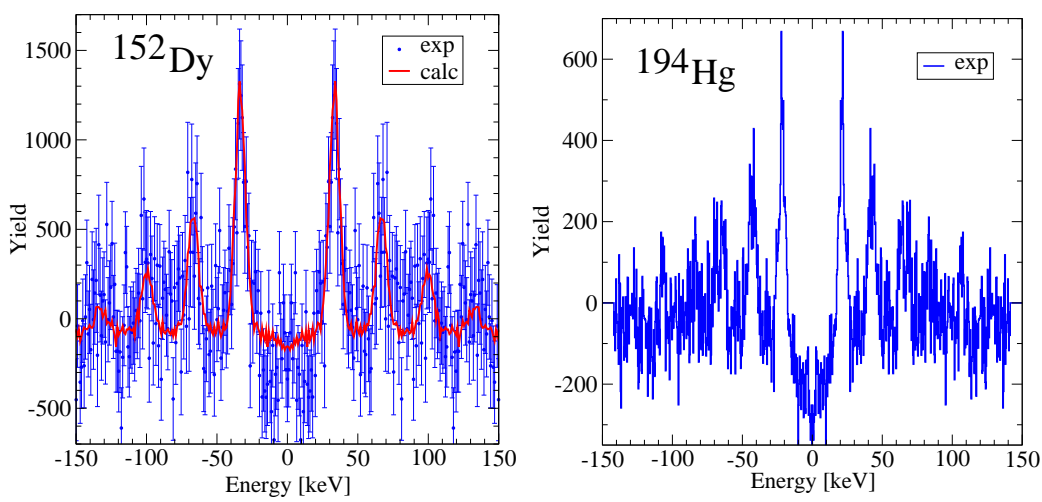

Figure 12: (Color online) Left panel) The ridges obtained when coincidence gates are placed on clean pairs of SD lines in ${ }^{152} \mathrm{Dy}$ [59]. At least four narrow ridges can be seen as well as a shallow valley. The solid line is from a Monte Carlo calculation of the ridges, see Sec. 4.1.1. Right panel) The ridges of ${ }^{194} \mathrm{Hg}$ extracted using the procedures described in the text. An independent extraction of the SD ridge in this nucleus was presented in Ref. [70].

the sensitivity to rotational correlations among discrete bands in the $\gamma$ cascades is largely enhanced as compared to a background of fragmented/uncorrelated decays. In fact, rotational correlation extending at least over three steps are selected, as shown in Fig. 13, where the $\mathrm{N}=1$ rotational plane pattern is schematically compared to the usual $\gamma-\gamma$ spectrum (assuming a regular rotational decay).

The method has been successfully employed in the analysis of a number of cases, from ND to SD nuclear systems $[69,72,73]$, allowing to quantitatively investigate the ridge structures both in terms of intensities and statistical fluctuations. In Sec. 4.1.3 results for the SD nuclei ${ }^{151} \mathrm{~Tb}$ and ${ }^{196} \mathrm{~Pb}$ will be discussed [73], while the potentiality of the method for searches of hyperdeformed, weakly populated structures [74] will be recalled in the Outlook section.
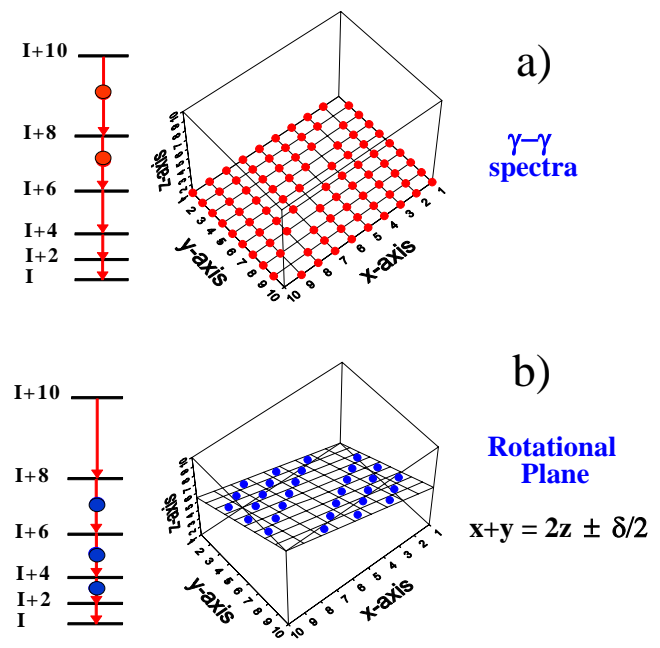

Figure 13: (Color online) Schematic illustration of the rotational correlation patterns following a regular rotational decay, for both a $\gamma-\gamma$ coincidence spectrum (panel a)) and the rotational plane defined by the equation $x+y=2 z \pm \delta / 2$, being $\mathrm{x}, \mathrm{y}$ and $\mathrm{z}$ the energies of the $\gamma$ rays, and $\delta$ the thickness of the plane (panel b), see text for details). The combination of coincident $\gamma$ rays selected by the two spectra are indicated by circles in the rotational cascades shown in the left hand side of each panel [75]. 


\subsection{Measuring the entry distribution}

The entry distribution defines the starting points of the $\gamma$ cascades in the spin-energy plane. Together with the end points into the yrast line, the initial points delineate the region in spin and excitation energy probed by the nucleus. Moreover, the experimental data are often compared to simulations of the $\gamma$ cascades. Thus, it becomes important to measure the total entry distribution.

The procedure for the GS array is described in detail in Ref. [59], but any $\gamma$-ray detector array with a good calorimetric response can be used using the same techniques. It consists of the following steps: (i) extraction of the experimental (detector fold, sum energy) distribution often called the $(\mathrm{K}, \mathrm{H}$ ) distribution; (ii) deconvolution of the $(\mathrm{K}, \mathrm{H})$ distribution with the detector response to obtain the experimental (photon multiplicity, total energy) distribution called (M,E) distribution; (iii) transformation of the (M,E) distribution to obtain the (spin, total energy) entry distribution of the nucleus.

\section{(i) Measuring the $(K, H)$ matrices}

Pairwise coincidence gates are placed on ND transitions in the nucleus of interest and the sumenergy $\mathrm{H}$ and the number $\mathrm{K}$ of detector modules that fire are recorded. For GS, a module is comprised of a Ge crystal and the seven BGO scintillator detectors that surrounds it. $\mathrm{K}$ is often called fold. The observed fold, K, and sum-energy, H, are sorted into 2D histograms, referred to as (K,H) matrices. The $(\mathrm{K}, \mathrm{H})$ matrices are then background subtracted using a modified version of the procedure described in Ref. [76]. This background subtracted $(\mathrm{K}, \mathrm{H})$ distribution is, however, not the true entry distribution as corrections for the response of the detector array must be applied first.

To measure the H (sum-energy) and K (module fold) efficiency, one uses a source, which emits $2 \gamma$ rays. At GS, the response is measured using a ${ }^{88} \mathrm{Y}$ source placed in the center of the array and acquiring data in singles mode. Events are subsequently selected by demanding that the photopeak of the $1836-\mathrm{keV}$ line is observed in one of the Ge detectors, hereby ensuring that whatever is detected in coincidence is the response of GS to the emission of a $898-\mathrm{keV} \gamma$ ray. There is a certain probability that this $898 \mathrm{keV} \gamma$ ray will be completely or partially absorbed in GS, or not absorbed at all.

Batches of such events from 1 to 100 are added up and records are kept, at each step in the batch, of the total energy observed and the number of modules hit [77]. Two-dimensional response functions for the energy $\mathrm{H}$ and fold $\mathrm{K}$ response are obtained in this way; see Figs. 10-11 in Ref. [59].

\section{(ii) Extracting the $(M, E)$ matrices}

To find which distribution of emitted total energy (E) and photon multiplicity (M) gives rise to the measured (H.K) distribution, a so-called "unfolding" of the measured (K,H) distribution is carried out following a Monte-Carlo-based procedure detailed in Refs. [77, 59]. This iterative procedure quickly converges towards a final, best approximation to the (M,E) entry distribution that folds into the measured $(\mathrm{K}, \mathrm{H})$ entry distribution. It is expected that the $(\mathrm{M}, \mathrm{E})$ distribution found in the Monte Carlo unfolding procedure is a fair representation of the $(\mathrm{M}, \mathrm{E})$ distribution that would be observed in an ideal calorimeter with $100 \%$ efficiency and infinite granularity of $\gamma$-ray detectors.

\section{(iii) Determining the entry distribution}

To arrive at the final 'spin - total - energy (I,E) distribution' for a reaction channel, the effect of any isomer tagging and/or isomers has to be taken into account and the multiplicity dimension of the measured 'multiplicity - sum-energy (M,E) distribution' must be translated into the associated (I,E) distribution. Fortunately, from the 1D QC analysis of Sec. 2.1.2, the mean spin removed per $\gamma$ is determined, thus, the multiplicity from the $(\mathrm{M}, \mathrm{E})$ can be converted to spin by multiplying with this 
experimentally determined factor [59, 78]. If there is any significant internal conversion for low energy $\gamma$ rays the entry distribution will have to be modified accordingly. Examples of internal-conversion correction can be found in $[79,80]$. Examples of measured entry distributions are shown in Fig. 14.
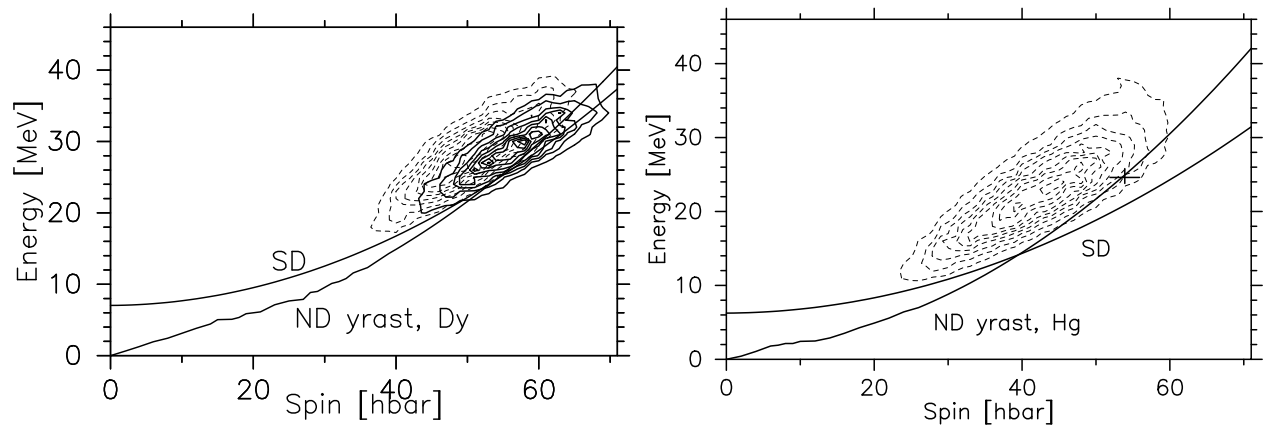

Figure 14: The ND and SD entry distribution for ${ }^{152} \mathrm{Dy}$ [59] (left) and ${ }^{194} \mathrm{Hg}$ (right) extracted using the procedures describes in section 2.2. For SD ${ }^{194} \mathrm{Hg}$ only the mean SD entry point has been extracted [60] and is shown as a ' + ' with errors.

\section{$2.3 \quad$ Fluctuation analysis}

Quantitative information on the average properties of the $\gamma$-decay flow can be extracted from the analysis of the fluctuations in the number of counts measured in the $\gamma$-ray spectra. In fact, when the $\gamma$ cascades, cooling the nucleus from the entry distribution to the yrast line, have an extremely large number of pathways to choose from (equivalent to infinite), the fluctuations of the data points in one-dimensional spectra, two-dimensional and higher order matrices follow 'normal' counting statistics. However, when the number of pathways available to the $\gamma$ cascades is finite, fluctuations above and beyond the statistical fluctuations can be observed and from that the number of pathways $N_{\text {path }}$, or effective number of transitions sampled in the decay, can be determined [71, 68]. $N_{\text {path }}$ is given by $\left(\Sigma_{t} w_{t}^{2}\right)^{-1}$, where $w_{t}$ is the probability that a particular transition is used in the cascade. This number should be evaluated within an energy interval of the order of the characteristic energy scale of the cascade: this can be either the energy difference $\Delta E_{\gamma}$ between rotational transitions or the temperature value, in the case of statistical decay-out or feeding transitions.

The study of the decay-out spectrum, discussed in Sec. 4.2 .3 in the case of ${ }^{192} \mathrm{Hg}$ [81], offers an example of fluctuation analysis in one dimension. In the particular case of the decay out, the fluctuation analysis method relies on the fact that below the excitation energy of the SD band-head, the level density in the ND well is large but nevertheless remains finite. This implies that the number of states available for the decay and hence the number of transitions that can be used in this process are also finite. If the number of events recorded in coincidence with the SD band is greater than this number of transitions, then more than one event will use the same transition. This will cause an enhancement of the fluctuations of the distribution of counts in the experimental spectra above those generated by pure counting statistics.

In the decay-out analysis, the number $n_{\text {path }}^{(2)}$ of transitions used in the decay can be related to the number of event $N_{\text {eve }}$, the variance $\mu_{2}$ and to the mean $\mu_{1}$ of the count distribution in the decay spectrum

$$
n_{\text {path }}^{(2)}=2 \times P \times f^{2} \times \frac{N_{\text {eve }}(A) \times \mu_{1}(A)}{\mu_{2}(A)-\mu_{1}(C)-g^{2} \mu_{1}(B)}
$$

where spectrum $\mathrm{A}$ is defined as $\mathrm{A}=\mathrm{C}-\mathrm{gB}$, being $\mathrm{C}$ the raw spectrum of all the events in coincidence with the SD band and B the appropriate background spectrum. Spectra A, B and C must be previously 
corrected for the detector response [50], as described in Sec. 2.1.2. The superscript (2) indicates that the extraction of the number of paths is based on first and second moments, while the $P$ factor corrects for the finite resolution of the detector system [68]. Correction factors are also added to account for Porter-Thomas fluctuations (the factor of 2), background subtraction and the separation of true coincidences into two components: the SD decay spectrum and the underlying continuous statistical feeding spectrum (the factor $f$ ) [68].

The most common application of the fluctuation analysis is related to the warm rotational motion, which is best analysed making use of two dimensional $\gamma-\gamma$ coincidence spectra, as discussed in Sec. 2.1.3. In two dimensions, a path $i \equiv\left(i_{1}, i_{2}\right)$ is defined as a given pair of coincident E2 transitions $\left(E_{\gamma_{1}}, E_{\gamma_{2}}\right)$, corresponding to a point in the $\gamma-\gamma$ plane. In particular, a path can belong to a discrete rotational band, populating the ridge structures, or it can be formed by a pair of damped transitions and lie in the diagonal region of the $\gamma-\gamma$ coincidence matrix. By estimating the number of paths, $N_{\text {path }}^{(2)}$, in these two different regions of the spectrum, one can learn about the properties of the rotational structures underlying the $\gamma$-decay flow, such as the density of states, the onset of rotational damping, the fragmentation of the $\mathrm{B}(\mathrm{E} 2)$ strength.

It is possible to show that

$$
N_{\text {path }}^{(2)}=\frac{N_{\text {eve }}}{\left(\frac{\mu_{2}}{\mu_{1}}-1\right)} P
$$

where $N_{\text {eve }}, \mu_{1}$ and $\mu_{2}$ are the number of events and the first and second moments of the distribution of counts in a sector $4 \hbar^{2} / \Im^{(2)} \times 4 \hbar^{2} / \Im^{(2)}$ wide (being $4 \hbar^{2} / \Im^{(2)}$ the average distance between consecutive rotational transitions), in a selected area of the $\gamma-\gamma$ spectrum. The moments $\mu_{1}$ and $\mu_{2}$ are found as

$$
\mu_{1}\left(x_{0}, y_{0}\right)=\sum_{x, y} \operatorname{Cor}(x, y) f\left(x-x_{0}, y-y_{0}\right)
$$

and

$$
\mu_{2}\left(x_{0}, y_{0}\right)=\sum_{x, y}(\operatorname{Cor}(x, y)-F i t(x, y))^{2} f\left(x-x_{0}, y-y_{0}\right)
$$

where $\operatorname{Cor}(x, y)$ is the COR subtracted matrix described in Sec. 2.1.4. $f\left(x-x_{0}, y-y_{0}\right)$ is a two dimensional normalized Gaussian weighting function and Fit $(x, y)$ is a polynomial fit to the local data, typically a $7 \times 7$ area of the matrix around the point of interest (in the case of a $4 \mathrm{keV} / \mathrm{ch}$ spectrum binning).

The fluctuation analysis method was originally developed and employed to study warm rotation in normal deformed nuclei $[68,82,83,84,85]$. Extensive investigations were performed also in connection with specific nuclear configurations, such as low-K/high-K structures for the study of the transition between order and chaos in the atomic nucleus [86, 87, 88, 89, 90]. At a later stage, taking advantage of high statistics experiments, the fluctuation analysis technique became a powerful tool for a quantitative investigation of superdeformed configurations as well $[72,91,92,73,75,93,94,70]$, the topic of this review paper.

\section{Theoretical considerations}

The elongated SD shape poses challenges to many of the basic concepts of nuclear physics. A central theme of the present review is the coupling between SD states and ND states, mediated by the barrier separating the two. Part of this physics has been intensely studied by the fission process, especially the occurrence of fission isomers and fission resonances connected to states of the SD minimum. For fission, the coupling widths and decay widths of individual states around the neutron separation energy can be investigated in considerable detail by neutron resonance reactions [6]. 
Such accurate probes do not exist for the SD rotational states. Instead, the SD states has the rotational frequency as an extra parameter. Going down from one angular momentum to the next along a SD band is like turning a handle, producing systematic changes in energies, barrier heights, electromagnetic decay widths and coupling matrix elements.

In addition to the rotational frequency one may study the influence of temperature, and especially the feeding and decay out of SD bands yield insight into ensembles of excited states of SD and normally deformed shapes. It may be illuminating to take a birds-eye view of the important physics issues, with increasing temperature as the guiding parameter.

The lowest lying discrete SD bands in each nucleus has a temperature $T=0$. Still at low temperature, one finds the excited SD bands which have sufficient intensity to be resolved. Most of the research on superdeformation has focused on these discrete resolved bands, characterising their structure by several physical quantities, such as the magnitude of the quadrupole moment, which quasiparticle states are excited, how do pairing and octupole correlations evolve with increasing rotational frequency. Of present interest are the intensities of the different transitions along the bands, especially $(i)$ at top of the bands at the highest angular momenta, where the band states are populated from states higher up in temperature, and (ii) at the bottom of the observed bands, where the decay out takes place through transitions to ND states.

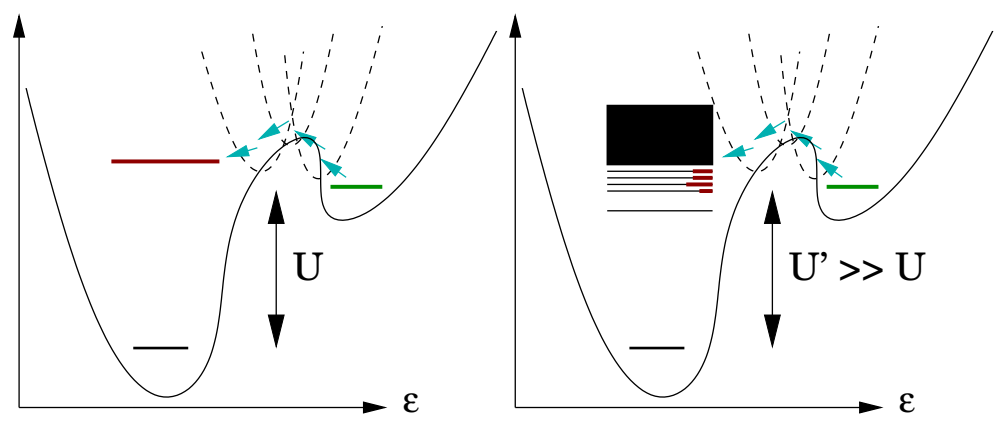

Figure 15: Schematic illustration of the coupling of SD states and ND states at an angular momentum where the SD well is rather shallow, and essentially only contains the lowest lying SD state. Left hand side: The SD state is connected through virtual barrier states to a doorway state on the ND side. Right hand side: The doorway state is further spread over many excited ND states, and it is the coupling to these states which eventually leads to decay out of the SD band. The parameter $\epsilon$ characterises the elongation of the nucleus.

Also, the barrier, which separates the SD band from the ND states is a low temperature phenomenon. The barrier states are not observed directly, but act as virtual states, through which the coupling between SD and ND takes place. This is schematically illustrated in Fig. 15. For the case of a high energy of the virtual barrier states combined with weak coupling matrix elements, the SD states are well shielded from the ND states at the other side of the barrier, even in cases when the level density of ND states is factors of 10 or 100 larger than the level density of SD states.

The concept of temperature implies averaging over several states, and it is actually meaningful to introduce temperature, even for resolved bands. Sampling in an experiment over decay cascades is in many respects equivalent to sampling over thermal canonical ensembles [95] This becomes especially relevant when the nuclear states are connected by transitions which are too weak or too close-lying to be resolved by present-day techniques for discrete $\gamma$-ray spectroscopy. Still, their transitions display correlations, and they can be investigated by QC spectroscopy, especially energy-energy correlations, such as described and illustrated in Sec. 2.1.4 of the present review. By these techniques one investigates an ensemble of states, and it is interesting to compare to the equivalent ensembles of ND bands. The elongated shape implies that excited SD bands display a smaller dispersion of rotational frequency 
among bands as well as a smaller dispersion in the moment of inertia. These are the prerequisites for the possible existence of ergodic bands [96].

Still higher up in excitation temperature, the rotational bands are mixed. This is a regime of compound nuclear states, and the important quantities become strength functions and level densities. These are the states relevant for describing the feeding into SD bands by the feeding QC transitions, and the feeding depends on the coupling between SD and ND states, mediated by the barrier.

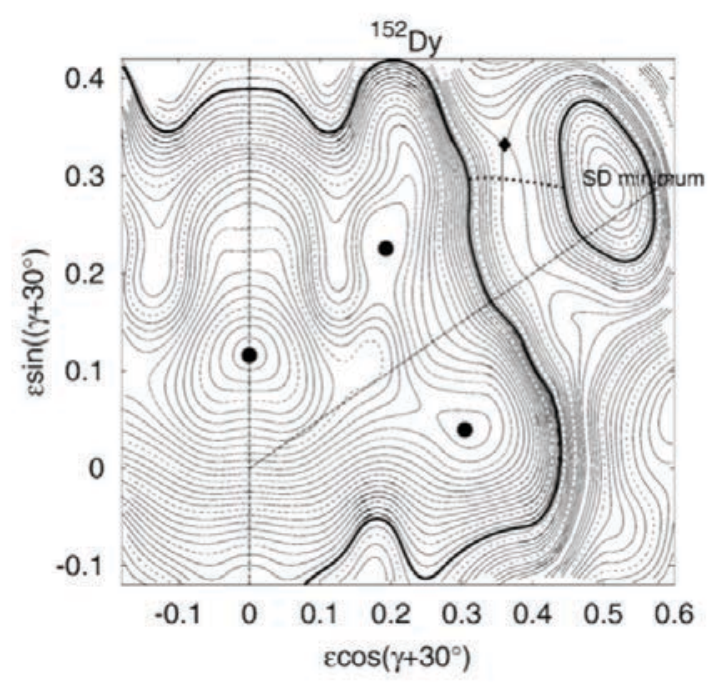

Figure 16: The calculated potential energy landscape in the $\epsilon-\gamma$ plane for the nucleus ${ }^{152}$ Dy at angular momentum and parity $I^{\pi}=40^{+}$. The interval between contours is $0.2 \mathrm{MeV}$. The energy contour at 1.5 $\mathrm{MeV}$ above the SD minimum is shown with a thick solid curve, and the least action path at this energy with a thick dashed curve. (from Yoshida et. al. [97]).

\subsection{Energy surfaces and barrier penetration}

The stability of the SD shape stems from the shell structure of single particle levels in deformed potentials. For a long time, it was thought that only the spherical shape carried substantial shell structure while the change in the energy of a nucleus with elongation of the nuclear shape was described by the liquid drop model. This simple view was changed with the introduction of the shell-correction method by Strutinsky and coworkers [1,98]. Especially, the shell-correction method could explain fission isomers, as well as the stable deformations for mid-shell nuclei. Later, the Strutinsky method was applied in large scale calculations of rotational nuclei, and it was predicted that nuclei in mass regions $A \sim 150$ and $A \sim 190$ would be SD in certain intervals of angular momentum [99, 100, 101]. Further insight into the shell structure, favouring a prolate shape with axis ratio $2: 1$, has been provided, based on the special degeneracies of a harmonic oscillator potential [102], alternatively by periodic orbit theory [103].

The special stability of SD states leads to a pronounced and rather sharp minimum of negative shell correction energy as function of deformation. When combined with a liquid drop energy which is weakly dependent on deformation, this minimum becomes a local or global minimum of energy as function of deformation. For the case of fission isomers, the weak dependence on deformation of the liquid drop energy is provided by the competition between surface tension and Coulomb repulsion. For $\mathrm{SD}$ rotational bands, the rotational energy combines with the Coulomb repulsion to counterbalance the surface tension, generating a rather flat liquid drop energy surface. 


\subsubsection{Pair-hopping model}

Fig. 16 from the work by Y. Yoshida et al. [97] displays an energy surface as function of two deformation coordinates, the $\epsilon-\gamma$ plane for the nucleus ${ }^{152} \mathrm{Dy}$ at angular momentum $40 \hbar$. The energy is calculated at each deformation from states of a cranked Nilsson potential, interacting with a volume- $\delta$ residual interaction. The most favourable deformation is an oblate ellipsoid $\left(\gamma=60^{\circ}\right)$ with axis ratio 0.9:1. Further, there exist two shallow triaxial minima, and a SD minimum, with $\gamma \approx 4^{\circ}$, that is close to an elongated prolate shape of axis ratio 2:1. The SD minimum is $2.7 \mathrm{MeV}$ above the oblate minimum. Further up in energy is the barrier, at $3.2 \mathrm{MeV}$ above the SD minimum.

On Fig. 15, it is indicated that underlying the energy surface are actual nuclear many-body states, and it may require several jumps in state to move from the SD to the ND minimum or vice versa. The ease or hindrance with which the nucleus can jump over the barrier depends on the interactions coupling the different states along the way. For an even-even non-rotating nucleus, the pairing interaction is very efficient in providing this coupling. For an odd-mass nucleus, the pairing is weaker, and the $K$-quantum number has to be conserved at jumps. Both of these effects lead to hindrance, yielding a factor of about $10^{-2}$ to $10^{-3}$ on the transmission coefficient [6]. For the SD rotating nuclei, an even-odd effect is not expected, but rather a general weakening of the pairing interaction. For rotating nuclei, at each deformation, the pairing gap is evaluated, and the mass parameter is scaled according to

$$
m_{i j}(q, I)=\frac{2 \Delta_{0}^{2}}{\left[\Delta_{n}^{(e f f)}\right]^{2}+\left[\Delta_{p}^{(e f f)}\right]^{2}} m_{i j}^{(h o p)}(q)
$$

The mass parameter is named "hopping mass parameter", because it arises from hopping from one configuration to the next over the barrier. Here, $\Delta_{0}$ is the average pair-gap of the non-rotating nucleus, and $\Delta_{n}^{(e f f)}$ and $\Delta_{p}^{(e f f)}$ are the Random Phase Approximation (RPA) pair-gaps evaluated at the precise angular momentum $I$, and deformation $q$ specified by the two coordinates $\epsilon, \gamma$. One can see that weakening of the pairing implies a larger mass parameter. The mass parameter has two indices for the two deformation coordinates, and it should be chosen such that it locally accounts for the density of pair crossings per unit deformation coordinate. This is roughly accomplished by relating the scale for changes in the coordinates to the three axis lengths of the volume conserving ellipsoid, (cf the description in reference [97]). Especially, around the spherical shape, the mass parameter is

$$
m_{i j}^{(h o p)}(q=0)=m_{0}^{(h o p)} \delta_{i j} \quad \text { with } \quad m_{0}^{(h o p)}=\frac{\hbar^{2} \Delta_{0}^{2}}{G_{0}} \frac{A^{2}}{27}
$$

where $G_{0}$ is the strength of the pairing interaction. The first factor of the basic hopping mass $m_{0}^{(h o p)}$ accounts for the ease with which the nuclear shape can jump from one state to the next, and the second factor accounts for the density of states per unit change of the deformation coordinates.

Further, the action integral for penetrating the barrier is evaluated by means of the usual WKB integral:

$$
S(E)=\int_{p a t h} d s \sqrt{2 m_{0}^{(h o p)}(V(q(s))-E)}
$$

where $V(q)$ is the potential energy surface. The tunnelling path $s$ minimises the action integral, and its metric relates back to the basic hopping mass:

$$
m_{0}^{(h o p)} d s^{2}=\sum_{i j} m_{i j}^{(h o p)}(q) d q_{i} d q_{j}
$$

With this metric, weak paring implies long tunnelling paths. Finally, the transmission coefficient for the barrier is given in terms of the action as:

$$
T=\frac{1}{1+\exp (2 S)}
$$


With this parameterisation, the abrupt decay out of the SD band over few steps in angular momentum may be related to a sudden increase of paring at barrier deformations with decreasing angular momentum [104, 105]. The abrupt decay out may alternatively be related to the onset of chaos-assisted tunnelling [38].

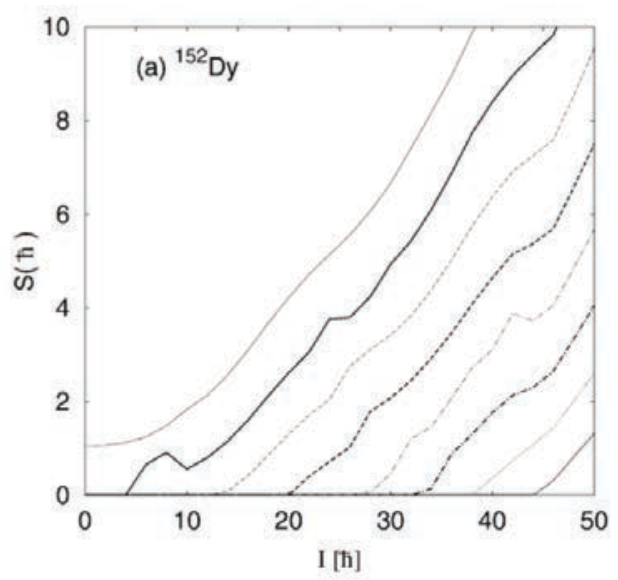

Figure 17: The tunnelling action $S(E, I)$ for crossing the barrier between ND and SD is plotted as function of spin for different excitation energies of the nucleus ${ }^{152} \mathrm{Dy}$ for even angular momenta and even parity. The thin solid curve with the largest action corresponds to the energy of the SD minimum, and then follow curves for energies up by $0.5,1.0,1.5 \cdots \mathrm{MeV}$. (from Yoshida et. al. [97])

Figure 17, from Yoshida et al. [97], displays the action $S$ as function of angular momentum, shown for different energies below the barrier. The figure displays a strong angular momentum dependence of $S$. For given energy above the bottom of the SD well, every two steps in angular momentum imply an increase of the transmission coefficient by a factor of about 1.6. Even more dramatic is the dependence with excitation energy. It only requires an increase in excitation energy of about $0.2 \mathrm{MeV}$ to increase the transmission coefficient by a factor of 2 .

\subsubsection{Generator coordinate method}

A more fundamental description of the nuclear many-body states at the different deformations is provided by the Generator Coordinate Method, GCM [106]. The GCM is a restricted shell model calculation, based on states which can be generated along a certain set of collective coordinates. In the present case, the most important collective coordinate is the elongation, which can be represented by the quadrupole operator. The states generated by GCM are essentially cold (temperature $T \approx 0$ ), resulting from minimising the energy of the selected shell model Hamiltonian plus the collective operators scaled by Lagrangian parameters. Subsequently, the same Hamiltonian is diagonalised in the basis spanned by the generated states.

Figure 18, from the work by P. Bonche et al. [107] displays as the continuous curves the potential energy at each value of the quadrupole operator. The final energy eigenstates are shown as horizontal lines, centred at the expectation value of the quadrupole operator. The barrier is rather shallow, and there is only one state in the SD potential well. The coupling implied by rotation has not been included in this investigation. Instead the energy of the rotating shape has been added for higher angular momenta.

Since the same basic Hamiltonian is applied all the way, the GCM method has the considerable virtue of being self-consistent - once the shell model basis states and the interaction is chosen, there are 


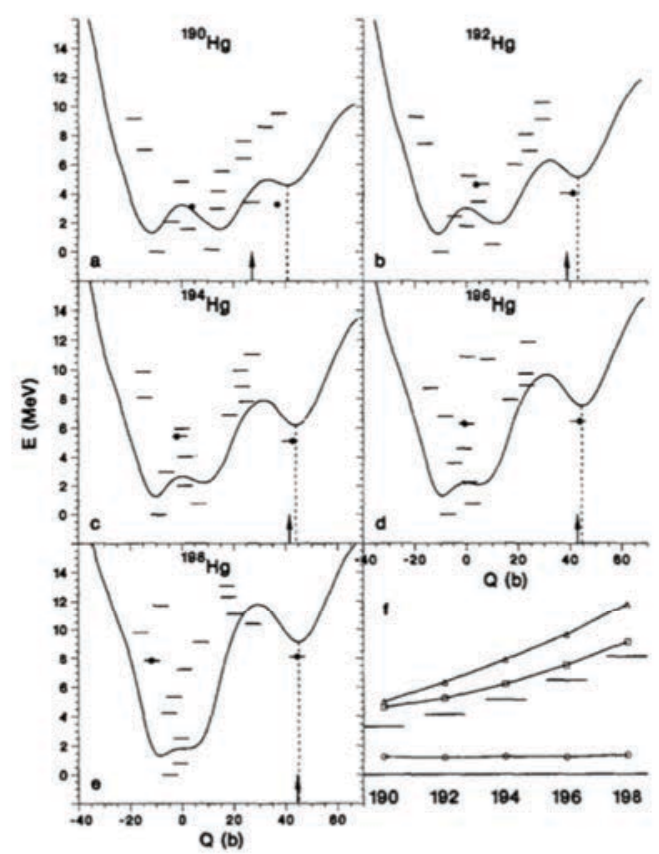

Figure 18: Solid curves: HF+BCS energy constrained with the quadrupole moment. Horizontal bars: GCM energies centred at the average quadrupole moment of the GCM eigenenergy. Solid dots denote the result of an extra diagonalisation of a $2 \times 2$ matrix of the quadrupole operator to produce a more SD specific state. Lower right corner: various quadrupole moments (not relevant for the discussion in the present review). (from Bonche et. al. [107])

no extra assumptions or parameters.

Applications of GCM to states in the ND and SD wells were later extended to include the octupole moment [108], thereby introducing states of negative parity. A more realistic picture of states arises, making it possible to evaluate E1, E2 and E3 matrix elements connecting states across the barrier. Also the technically demanding inclusion of angular momentum in GCM descriptions of ND and SD states with triaxialy deformed quadrupole shapes has been carried out [109].

In general, the different applications of GCM to $\mathrm{Pb}$ and $\mathrm{Hg}$ nuclei yield similar results with regard to the barrier heights, which tend to be about $1 \mathrm{MeV}$ above the lowest SD state around angular momentum 6 to $12 \hbar$, where the decay out takes place experimentally. Decaying down excited bands from high angular momentum, the barrier may disappear for some of the excited SD states which in this way dissolve below a certain angular momentum [109], but the decay out will take place before that by means of transitions down to ND states. In detail, papers on GCM have somewhat different views on the decay-out mechanism of the lowest-lying SD band, which is still rather well shielded by the barrier at decay out. We find it instructive to present the discussion of decay out from reference [107], since it is consistent with the basic setup of deformations and wavefunctions in the GCM.

However, as a minor digression, and to set the early work on the GCM in perspective, it should be mentioned that GCM techniques subsequently have been developed to higher precision and generality. Especially, the GCM has recently been very successful in describing the occurrence of static and dynamic deformations for all nuclei throughout the periodic table [110].

Conceptually, the states generated by the GCM can be viewed as collective states in the quadrupole coordinate, and Fig. 19 from P. Bonche et al. displays wavefunctions. The left hand side shows the two lowest states in the ND well, the ground state without a node and the first excited state carrying one node. On the right hand side are shown the wavefunctions of predominately SD nature, and the ND state to which it couples, typically the 3'rd to the 6'th excited state. For these states, an extra 


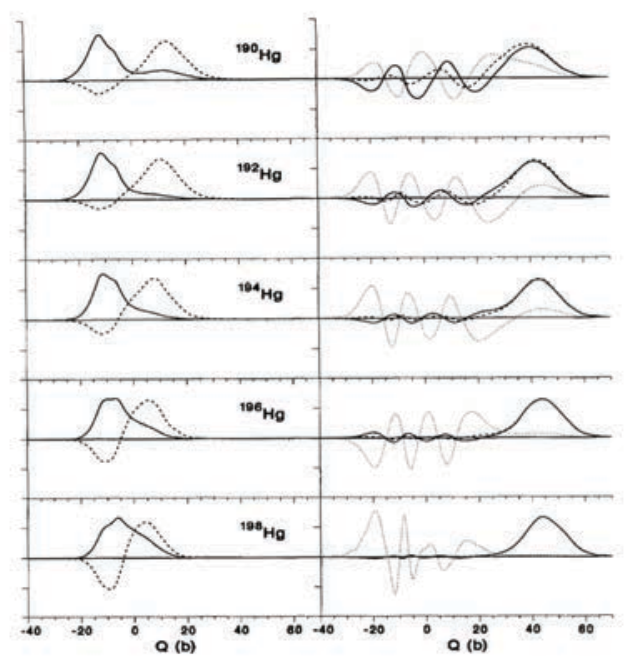

Figure 19: GCM Wavefunctions in the quadrupole coordinate. Left-hand side: the ground (solid) and first excited states (dashed). Right hand side: the same information for the SD state (solid), its nearest neighbour state (dots), and the SD state after the extra diagonalization of the quadrupole operator (dashed). (from Bonche et. al. [107])

diagonalisation of the quadrupole operator has been carried out. This can be justified by the presence of the barrier, and it represents a decoupling of the states of the two wells, relevant for studying the decay of SD bands. Within the basis spanned by the GCM states, the SD state is connected by E2 transitions of high energy down to low lying ND collective quadrupole states, competing with the strong rotational transitions along the band. Eventually, at a sufficiently low angular momentum, the transitions down into the ND states will win, and take the nucleus out of the SD well. This is displayed in Fig. 20. As argued by P. Bonche et. al., these transitions carry substantial strengths, by which the nucleus should decay out of the SD band down to rather low lying states, even if the ND collective states are spread over several states.

\subsection{Coupling to compound states}

The actual decay observed, as described in the other sections of the present review, shows that the lowest SD states mainly decay by highly fragmented cascades, with the first transition going to states of rather high excitation energy above yrast on the ND side. This is reminiscent of statistical cascades following neutron capture via compound resonance states at low neutron energy.

The GCM states only describe a small part of the actual state space in nuclei, and one must supply the states displayed in figure 18 by a dense spectrum of many-particle many-hole states, which may mix among themselves, and to which the GCM states will couple. The GCM doorway states with many nodes are viewed as many phonon vibrations in the ND well. Both particle states and hole states entering into these vibrational wave functions may spread on more complicated states, eventually leading to damping of the vibration.

The model for describing "Chaos assisted tunnelling" by S. Aaberg [38] starts out with a pure SD state and a pure ND doorway state, coupled by a barrier transmission matrix element. On the ND side, the doorway state is part of a spectrum of states, and the approach towards chaos is modelled by increasing the strength of the off-diagonal matrix elements within all ND states. This leads to an increasing damping width of the doorway state, as well as an increasing amplitude of ND content in the predominately SD state. The $\gamma$-decay amplitude of this admixture of ND states cause the decay out of the SD state. Full chaos assisted tunnelling is achieved when the ND states are described by a full 


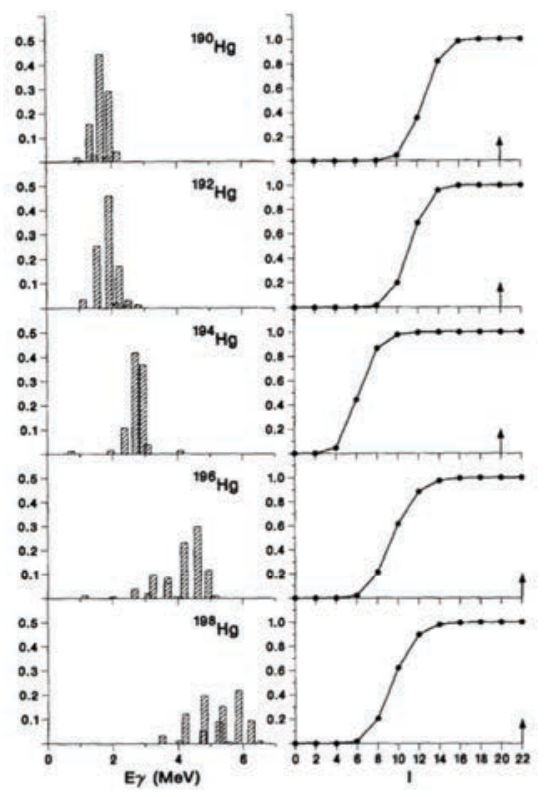

Figure 20: Decay of the SD band for five Hg-isotopes, according to GCM wavefunctions. Left hand side: relative intensities of the decay-out E2 transitions. Right hand side: The evolution of the population of the SD band - displaying the decay-out pattern. (from Bonche et. al. [107]).

Gaussian Orthogonal Ensemble (GOE) and the doorway state is spread over the whole ND spectrum. In the actual ND well, this situation occurs when the doorway states are mixed over intervals which are of the same order or larger than the energy distance between them.

Our expectation of the situation at feeding and decay out of the SD band may again be inspired by the situation for Actinide nuclei, investigated by the fission process. Wide as well as narrow resonances of fission of Actinide nuclei can be understood on the basis of structure of states in the SD well alone, with no need for structure of the states in the first well [6]. As a result, the whole ND side is represented just by the decay widths and the level density of ND compound states and the barrier separating ND and SD states. And it is argued by Bjørnholm and Lynn that multiphonon states at the relevant energies in the ND well, that is around 5-6 MeV of excitation energy, must be spread over large energy intervals.

In the following we discuss this picture of full mixing of doorways into a compound ND spectrum, although it should be stated that at SD decay out, the excitation energies are lower than the relevant excitation energies for fission, and one cannot take full compound mixing for granted.

The situation at feeding is equivalent, only now also the SD excited collective state should be coupled over compound states in the second well. Following the notation of Bjørnholm and Lynn, compound states are denoted by $|\lambda\rangle$ and doorway states by $|\nu\rangle$, and the coupling between compound states are mediated by the nearest lying doorway states:

$$
\left\langle\lambda_{S D}|H| \lambda_{N D}\right\rangle \approx\left\langle\lambda_{S D} \mid \nu_{S D}\right\rangle\left\langle\nu_{S D}|H| \nu_{N D}\right\rangle\left\langle\nu_{N D} \mid \lambda_{N D}\right\rangle
$$

With the doorway states spread over intervals typical of their separation, and the average mixing of 
doorway states into the compound states around them attains the magnitude

$$
\overline{\left\langle\nu_{N D} \mid \lambda_{N D}\right\rangle^{2}} \approx \frac{D_{N D}}{\hbar \omega_{N D}}
$$

Where $D_{N D}$ denotes the level spacing of compound states, and the level spacing of doorway states is represented by the vibrational frequency $\hbar \omega_{N D}$ in the ND well, and equivalently for the SD well.

At the collective level, these frequencies give the time scale, and if the doorway states would be on their own, without the compound states, one would obtain for the coupling width of the SD doorway to the closest ND doorway in energy:

$$
\Gamma_{S D \rightarrow N D}(\text { doorways })=\frac{\hbar \omega_{S D}}{2 \pi} T
$$

$T$ being the transmission coefficient.

With spreading over the compound states, as described schematically by the matrix elements (Eq. 15) and (Eq. 16), the coupling width for SD and compound ND states is governed by level spacings in the two wells:

$$
\Gamma_{S D \rightarrow N D}=\frac{D_{S D}}{2 \pi} T
$$

Likewise, the coupling from ND to SD is governed by the same expression, replacing "ND" by "SD", and vice versa. Altogether, these considerations imply that realistic descriptions of feeding and decay out require careful evaluation of the level densities on both sides of the potential barrier.

According to some descriptions of SD feeding and decay out, these spreading widths for coupling are interpreted as time scales, implying fast times for hopping back and fourth between the wells $[111,112,113]$ or fast decay-out times [114]. However, this interpretation is only valid for decay to a true continuum of states, and this will not be the case for SD feeding and decay out.

Theoretical calculations as well as evaluations of the transmission coefficient are often characterised by two parameters $(i)$ the barrier height $V_{B}$ and $(i i)$ the effective frequency at the barrier, $\omega_{B}$. These two parameters refer to the simplest approximation of the potential energy and mass parameter around the top of the barrier. For an inverted parabola of potential energy and a constant mass parameter, the transmission coefficient reads:

$$
T=\frac{1}{1+\exp \left(2 \pi \frac{V_{B}-E}{\hbar \omega_{B}}\right)}
$$

Where $E$ is the energy, so the energy $V_{B}-E$ measures the height of the part of the barrier which has to be hopped over. This expression has been widely applied in fission and SD decay-out studies. For example, the barrier displayed in Fig. 16, has a barrier height $V_{B}=2.67 \mathrm{MeV}$ measured relative to the bottom of the SD well, and a barrier frequency of $\hbar \omega_{B}=1.03 \mathrm{MeV}$. With decreasing angular momentum, corresponding to going down a decay cascade, the barrier height decreases and the barrier frequency increases, the latter implied by the increasing pairing gap.

In fission, the coupling widths for excited states in the second well and decay widths to fission have been investigated by neutron induced fission, and a fine example is displayed in Fig. 21.

In this case, the average spacing between SD states is about $450 \mathrm{eV}$, and the average spacing between ND states is about $15 \mathrm{eV}$. From the resonances, one extracts an average coupling width of $\Gamma_{S D \rightarrow N D} \approx 2 \mathrm{eV}$, implying that each $\mathrm{SD}$ remains fairly pure, with a fission width of around $2 \mathrm{eV}$, with some spreading to 3 or 4 ND neighbouring states, which acquire fission widths of the order of 10 $\mathrm{meV}$. The double resonance around $1400 \mathrm{eV}$ is an exception, arising from near degeneracy between the SD compound state and one ND compound state. From the transmission coefficient, one may infer the barrier height from Eq. 19, expressed as $2 \pi \frac{V_{B}-E}{\hbar \omega_{B}}=3.8$. In between the SD states, relatively wide 

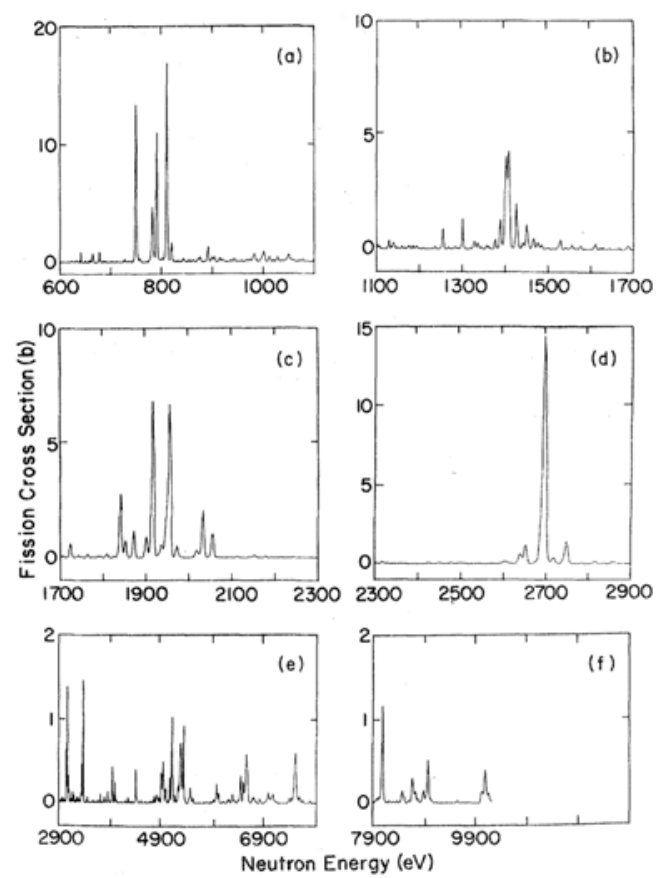

Figure 21: ${ }^{240} \mathrm{Pu}$ subthreshold fission cross section from 500 to $10000 \mathrm{eV}$. The panels show how the resonances with the largest fission cross section cluster around excited states in the SD well, which act as doorway states for fission. (from Auchampaugh and Weston [115]).

intervals in energy, containing the bulk of ND states, are rather unaffected by the presence of the SD states.

Inferring from fission, the situation on Fig. 21 serves as an illustration of the situation at feeding of the SD states at about $V_{B}-E=0.5 \mathrm{MeV}$ below the top of the barrier.

Assuming that the level density in the SD well is smaller than in the ND well, as is usually the case, the states of the SD well will only couple locally, as displayed on Fig. 21, and with the SD electromagnetic decay matrix elements larger than those of ND, once the nucleus has populated a SD state of excitation energy of about 0.5 below the barrier or lower, the subsequent transitions will be of SD nature: the nucleus is "trapped in the second well". Going up in energy above the barrier, one eventually expects full mixing over an open barrier, implying that there should be no selection of the content of SD or ND in each compound eigen-energy state, just given by random coupling. However, this is not what the expression (18) seems to predict. Even above the barrier with the transmission coefficient $T \rightarrow 1$, one sees that a typical SD state is spread over an interval $1 /(2 \pi)$ times the distance to the next SD state. In between these neighbouring SD states, there will be states of fairly pure ND content. Thus, according to this description, full mixing of the states across the barrier cannot be achieved. Full mixing must then imply that additional doorway states, that is excited states in the barrier region, come into play. Eventually the coupling through several doorway states will lead to full mixing, which can more intuitively be called "opening of the barrier". We expect the open barrier to set in at energies just above the size of the pair-gap at the barrier, that is from about $1 \mathrm{MeV}$ from the top of the barrier in the mass 150 and 190 regions. 


\subsection{Level densities in both wells}

Systematic knowledge of nuclear level densities stem from (i) counting states at low energies and (ii) again counting levels in a narrow interval just above the neutron separation energy. Over recent years, a successful method connecting these two energy intervals is provided by the Oslo method [116].

Underlying all descriptions of the nuclear level density are many-particle-many-hole excitations of a one-body shell model potential. A basic understanding of nuclear level densities is provided by the Fermi gas model. For given neutron number $N$, proton number $Z$ and the excitation energy $E$, one obtains the following Fermi gas level density [117].

$$
\rho(N, Z, E)=\frac{\sqrt{\pi}}{12} \frac{1}{(a E)^{1 / 4}} \frac{1}{E} \exp (2 \sqrt{a E})
$$

where the level density parameter $a$ is a measure of the density of single particle levels $g_{n}$ and $g_{p}$ around the Fermi energies: $a=\frac{\pi^{2}}{6}\left(g_{n}+g_{p}\right)$. The energy $E$ should be the energy available for particle excitation, that is the energy above yrast [118]. The many-particle-many-hole states are excited in a cranked potential, and each state, as being counted in the level density (Eq. 20) is a member of a rotational band of a certain signature, that is the distance in angular momentum between states on the band is two units. When one in addition specifies the parity, there are four parity-signature combinations, and the cranking level density acquires a factor $\frac{1}{4}$ relative to the level density just as function of $N, Z, E$. And this is then the Fermi gas level density which is relevant to the SD and ND wells:

$$
\rho_{(\text {cranking })}(N, Z, E, I, \pi)=\frac{\sqrt{\pi}}{48} \frac{1}{(a U)^{1 / 4}} \frac{1}{U} \exp (2 \sqrt{a U})
$$

where

$$
U \equiv E-E_{\text {yrast }}
$$

is the heat energy, that is the energy available for thermal excitation of nucleons, while most of the energy is tied up in rotational energy, given by the energy of the yrast line $E_{\text {yrast }}$.

Level densities of nuclei close to their ground states and around the neutron separation energy are characterized by a level density parameter of about $a \approx \frac{A}{12}$, whereas the pronounced shell structure of the SD well states lead to a much smaller level density parameter of $a \approx \frac{A}{17}$.

It is important to point out the difference between the cranking model level density and the level densities investigated at low angular momenta, as counted, at the neutron separation energy, or investigated by the Oslo method. For level densities at low angular momentum, one adds the angular momentum projection as an additional parameter [117]. For spherical nuclei, the axis for the angular momentum projection is an external axis, and the level density as function of $(N, Z, E, I, \pi)$ is obtained by differentiation with respect to the projection $M$, this leads to the so-called Bethe formula for the level density. For deformed nuclei, the axis is the symmetry axis of the potential, the levels are intrinsic states of the deformed potential, and the level density is obtained by counting the number of levels obtained by building a rotational band on top of each intrinsic band, leading to the Ericsson formula for the level density. For low angular momenta $I$, the level density is approximately proportional to $2 I+1$, as one would expect for simple phase space considerations. The numerical calculations of states in Sec. 4 of the present review also apply the cranking model for the basis bands, and the cranking model level density (Eq. 21) will be the relevant one to compare to.

\subsection{Decay out of superdeformed bands}

At decay out of the lowest lying SD band, the SD state is interpreted as the lowest lying vibrational state on the SD side. This corresponds to the situation depicted by the GCM calculations on Fig. 18. 
This gives the energy scale $D_{S D}=\hbar \omega_{S D}$, and the state is at energy $\frac{1}{2} \hbar \omega_{S D}$ above the bottom of the well.

Vigezzi et.al. [119] applied the formulation of coupling to doorways to compound states outlined in Sec. 3.2 to the decay out of SD bands, supplying the SD state with the E2 rotational decay along the band and the ND states with statistical decay widths.

At decay out, for the mass 150 and 190 regions, the statistical $\gamma$-decay width of ND compound states is larger than the width for $\gamma$-decay along the SD band. Therefore, even a rather weak mixing of the closest ND state into SD is sufficient for decay out of the SD band. In this case, one obtains for the average decay-out probability:

$$
\bar{P}_{\text {out }} \approx \sqrt{\frac{\pi}{2} \frac{\Gamma_{S D \rightarrow N D} \Gamma_{\gamma, N D}}{D_{N D} \Gamma_{\gamma, S D}}}
$$

where the decay widths are denoted $\Gamma_{\gamma}$

For decay by E2 transitions down a superdeformed band, the average decay-out probability will increase, since both factors of the numerator of equation (23) will in general increase, and both factors of the denominator will decrease. On top of this, the random placement of the energy spectrum of ND compound states relative to the SD band state implies fluctuations of the decay-out probability $[119,120]$, which provide an explation of the jagged look of the curves of the intensity of SD transitions, at the angular momenta at decay out, as for example displayed for the mass 150 region in figure 4 of reference [121]. This supports the notion of decay through coupling to a compound spectrum. The later observation of the statistical decay-out spectrum by Henry et al. [122] has confirmed this picture. A further confirmation of the statistical nature of the decay-out spectrum has been obtained by evaluating the fluctuations of the spectrum [94].

Still, there is a limitation to this description, namely that the states are basically treated as stationary states. Actually the decay widths should be included in a consistent description. Descriptions including decay widths in a consistent way have later been formulated for the case of decay out of SD bands by $\mathrm{Gu}$ and Weidenmüller and by Cardamone et al. [123, 120]. Fig. 22, taken from the work by Gu and Weidenmüller, shows the result of including decay widths - basically as imaginary parts to the energies. The figure actually shows the opposite of the decay-out probability, namely the probability for staying in the band. Going through the panels with increasing value of $\Gamma_{\gamma, N D} / D_{N D}$, corresponds to going from discrete ND states towards overlapping resonances of ND nature.

$\mathrm{Gu}$ and Weidenmüller provide thorough explanations [123] of the effects of including the decay widths in the consistent form. Especially, for overlapping resonances, $\Gamma_{N} / D_{N D}>1$, the ND states effectively generate a continuum, to which the SD state couples with decay width $\Gamma_{S D \rightarrow N D}$, directly competing with the decay along the SD band. In this case, The probability for decay out is simply the branching ratio determined by these two decay widths:

$$
P_{\text {out }}=\frac{\Gamma_{S D \rightarrow N D}}{\Gamma_{S D \rightarrow N D}+\Gamma_{\gamma, S D}} \quad \text { for } \Gamma_{N, \gamma} / D_{N D}>1
$$

Both of the two lower right panels of Fig. 22 display results for this situation of overlapping resonances, and indeed the solid curves in these two panels are very similar, while the dashed curves show that the expression 23 drastically overestimates the decay-out probability in the case of overlapping resonances.

For SD decay out, decay by $\gamma$ rays, the resonances are far from overlapping, the ratio $\Gamma_{\gamma, N D} / D_{N D}$ being of the order of $10^{-3}$ for the actual cases of decay out from SD bands in the mass 150 and 190 regions. 

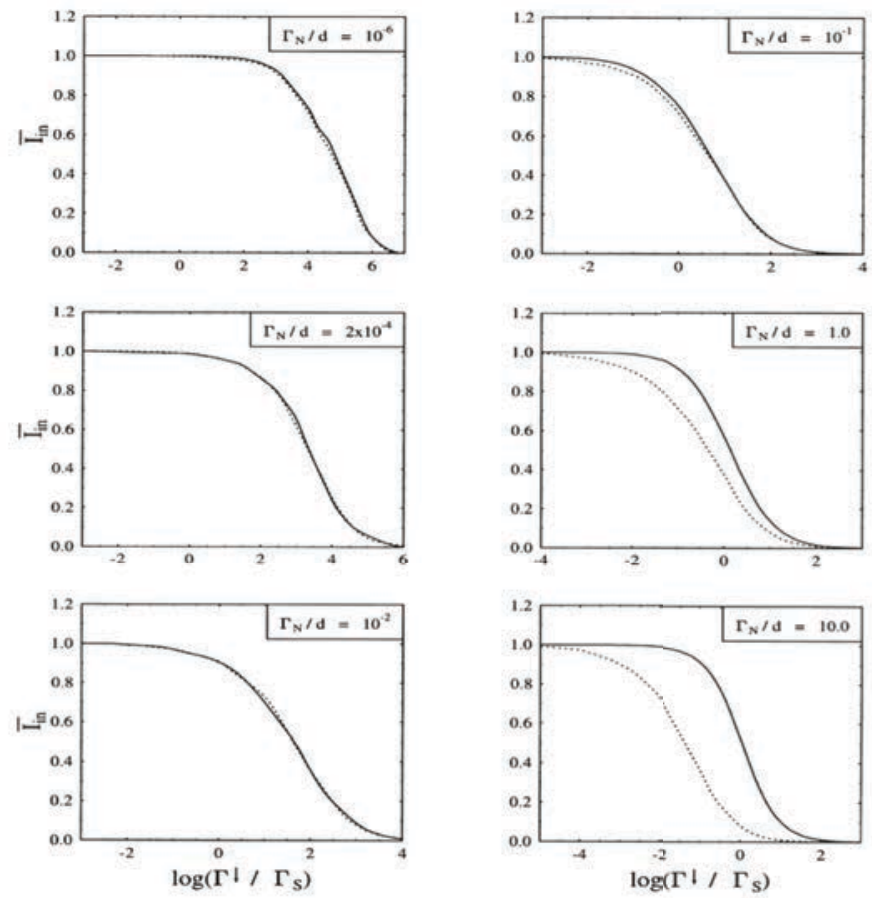

Figure 22: The ensemble-averaged total intraband intensity is shown as function of the ratio between the tunnelling width $\Gamma_{S D \rightarrow N D}$ (denoted $\Gamma_{\downarrow}$ in the figure) and the SD decay width $\Gamma_{\gamma, S D}$. The different panels are labelled by the ratio between the ND $\gamma$-decay width and the spacing between ND states. The upper left corner corresponds to very isolated resonances, whereas the lower right corner corresponds to strongly overlapping resonances. The result of including the decay widths in a consistent way (Gu and Weidenmüller) in the calculations are shown as solid lines, whereas the result of treating the states as stationary and then adding decay widths at the end (Vigezzi et al.) is shown as dashed lines. (from $\mathrm{Gu}$ and Weidenmüller [123])

Assuming a compound nature of the ND states, the SD state will couple with randomly selected matrix elements to the neighbouring ND states in energy. The coupling to the closest lying ND state will then be most important, and a two-level model is then quite realistic, as described by Cardamone, Stafford and Barrett [124, 120]. By carefully investigating lifetimes and branching to direct linking transitions, Kruecken et. al. [125] provided convincing support for the compound ansatz for the ND states. The ND state, which is mixed into the SD band carries very small decay matrix elements to the local yrast or yrare ND states, and this is characteristic of a strength function spread over states with a high level density. Also, based on this information, and based on estimates of the level density in the ND well, Kruecken et. al. [126] determined the coupling width $\Gamma_{S D \rightarrow N D}$ at decay out. Assuming standard values of the frequencies in the second well and of the curvature at the barrier, $D_{S D} \approx \hbar \omega_{S D} \approx \hbar \omega_{b} \approx 0.5$ $\mathrm{MeV}$, one can extract values for the different quantities characterizing the motion across the barrier:

$$
\begin{array}{lllll}
{ }^{194} \mathrm{Hg}, I=12: & \Gamma_{S D \rightarrow N D} \sim 50 \mathrm{meV} & T \sim 6 * 10^{-7} & S \approx 7.1 \hbar & V_{B}-E_{S D} \approx 1.1 \mathrm{MeV} \\
{ }^{194} \mathrm{~Pb}, I=6: & \Gamma_{S D \rightarrow N D} \sim 5000 \mathrm{meV} & T \sim 6 * 10^{-5} & S \approx 4.8 \hbar & V_{B}-E_{S D} \approx 0.8 \mathrm{MeV}
\end{array}
$$

where $T$ is the transmission coefficient, $S$ is the action. These values will carry an uncertainty of one to two orders of magnitude for the transmission coefficients and $\pm 0.5 \mathrm{MeV}$ for the barrier.

A somewhat different approach to the barrier dynamics has been given by Barrett et al., combining the two-level model with an asymptotically exact quantum tunnelling interaction. [127, 128]. The results are in overall agreement concerning the actions $S$ from Eqs. 25 and 26, whereas the terminology is different concerning other quantities. 
The values (Eqs. 25 and 26) of the barrier heights are in quite good agreement with different theoretical estimates, such as from references [97, 109]. All together for the mass 150 and 190 intervals, a consistent picture emerges of the decay out of the SD band by admixture of a small component of a compound ND state. Even stronger support for the compound nature of the ND state at decay out is provided by investigations of the QC decay-out spectrum, as presented in Sec. 4.2 of the present review.

For lighter mass regions, the SD deformation may be smaller, the barrier may be more shallow, there will be fewer states on the ND side, and doorways may be explicitly visible, as for example observed in the nucleus ${ }^{59} \mathrm{Cu}[129]$.

\subsection{Feeding of superdeformed states}

The hot rotating nucleus formed in fusion reactions first decays by evaporation of nucleons or $\alpha$ particles, and $\gamma$ cascading sets in when the nucleus has cooled down to an energy above yrast which is smaller than the separation energy of the particles. Still, particle evaporation would be possible if the particle carries away rotational energy, but this involves crossing large centrifugal barriers, severely cutting down the decay width for particle evaporation. This picture of the transition from particle evaporation to $\gamma$ emission is supported by detailed calculations, and is well in accordance with the average excitation energies above yrast of 4 to $6 \mathrm{MeV}$ for the $\gamma$-cascade entry displayed in the distributions of Fig. 14 .

At the angular momentum for entry, the height of the barrier separating the SD and ND potential wells is about 3 to $4 \mathrm{MeV}$, and the SD well is deeper than the ND well by about 1 to $2 \mathrm{MeV}$. As we have argued in connection to the illustration from fission, Fig. 21 the barrier provides a separation between the SD and ND states for the lower part of the entry distribution, about 2 to $3.5 \mathrm{MeV}$. On the contrary, at the higher part of the entry distribution, about 5 to $8 \mathrm{MeV}$, the SD and ND states are completely mixed across the barrier, mediated by many states in the barrier region, yielding similar total $\gamma$-decay widths of all states to lower energy SD or ND states. The $\gamma$ decay can then either be a rotational transition, or a statistical cooling transition, the latter cooling the nucleus by an energy of about 5 times the excitation temperature [95]. After this first cooling transition, the nucleus will typically have an energy below the barrier, and is then "trapped" in one of the wells.

Within the respective wells, the competition between statistical E1 cooling transitions and E2 rotational transitions will be in effect. For the SD well, the low level density implies fewer final states for the cooling transitions, which leads to a stronger feeding of the SD yrast band than the equivalent feeding of ND yrast states [95], as displayed in Fig. 29.

A probe of the SD feeding mechanism and the mixing between ND and SD states may be the feeding times for the resolved SD bands. This subject was taken up by Schiffer et. al [113]. If the feeding goes through highly mixed states, one expects rather long times for the cascade preceding the feeding into low lying SD bands, and this was predicted in reference [113]. However, as argued above and illustrated by the example from fission on Fig. 21, the barrier is probably more efficient in separating the SD and ND states than described in reference [113], and the feeding should be fast. Considering the short decay times for these transitions, it is not an easy task to extract information from the feeding times, and, in our view, this topic has not been addressed in analysis of experiments in a consistent way.

\subsection{Damping of superdeformed rotational motion}

After a nucleus is trapped in the SD well, the $\gamma$ decay arises out of competition between E2,E1 and M1 transitions, yielding information about strength functions of quite different origin. Especially, the distribution of energies of E2 transitions are determined by the degree of damping of the collective rotational motion of the elongated SD shape.

The traditional mass region for studying rotational motion is the rare-earth nuclei, mass number 165185. They are deformed - with axis ratio of about 1.3:1. For rare earth nuclei, experiments performed by 
Stephens, Diamond and coworkers first established the E2 nature of rotational cascades to the highest angular momenta, where resolved bands had not been observed [130]. Then studies of $\gamma-\gamma$ energy correlations performed by Herskind and coworkers showed that the rotational transitions for the major part of the cascades are not emitted as transitions along discrete rotational bands [66]. This implies that the rotational motion is damped, that is the frequency distribution for each state is smeared out by a characteristic energy, named the rotational damping width $\Gamma_{\text {rot }}$. The first theory of rotational damping was given by G. Leander [131], and a more comprehensive evaluation was given by B. Lauritzen et. al. [132]. According to this description, basis bands, as described by the cranking model, interact via the residual two-body interaction, thereby generating mixed band states. More detailed studies by $\mathrm{M}$. Matsuo and coworkers showed that the high-multipole part of the residual interaction is responsible for the band mixing $[133,134]$. This may be contrasted to the low-multipole part of the interaction, which produces correlated states, such as the pairing condensate (multipole 0), and the low-lying vibrational states (multipole 2 and 3).

The phenomenon of rotational damping in nuclei is characterised by several energy scales, which vary in specific ways as function of excitation energy, deformation, mass number and rotational frequency. These energy scales are generally expressed as power laws as functions of angular momentum $I$ and heat energy $U$ (Eq. 22). The density of states connected by the two-body residual interaction grows as $U^{3 / 2}$, that is the heat energy to the $3 / 2$ 'th power, and the width $\Gamma_{\text {comp }}$ (compound damping width) over which each basis band state spread is predicted to increase in proportion to $U^{3 / 2}$.

The rotational damping width itself is given by the dispersion in rotational frequency of bands mixed together in the energy eigenstates, and increases rather slowly with $U$ to the $1 / 4$ 'th power, as well as linearly with the angular momentum. However, the mixing of more and more bands with increasing excitation energy eventually will diminish the dispersion in rotational frequency. This is the phenomenon of motional narrowing, which is been described in other branches of physics, such as magnetic resonances (see Ref. [135] for a comparison between the line widths observed in nuclear magnetic resonance and the properties of rotational damping in nuclei).

Along the decay cascades, the nucleus cools, as on the average described by the flow line of average energy as function of angular momentum, $U \propto I^{2}$ [95]. This will lead to the following behaviour of observed rotational damping width:

$$
\left.\Gamma_{r o t} \propto U^{1 / 4} I \quad \rightarrow \quad I^{3 / 2} \text { on flow line (low } I\right)
$$

below the onset of motional narrowing. This should be the situation at low angular momenta, and thereby low average heat energies. Above the onset of motional narrowing, one obtains:

$$
\left.\Gamma_{r o t} \propto U^{-1} I^{2} \rightarrow I^{0} \text { on flow line (high } I\right)
$$

which should describe the situation at higher angular momenta.

In addition to the wide smearing out of rotational energies, given by $\Gamma_{\text {rot }}$, more narrow correlations in energy also exist for the mixed bands, forming the so called narrow component of the two dimensional strength function for consecutive transitions. The narrow component is generated by fluctuations in the transition strengths [136], it is wider than ridges stemming from unmixed bands, and it fades away with increasing excitation energy in inverse proportion to the number of basis states being mixed together, that is basically inversely proportional to the level density. For unmixed bands, the width of the first ridge is determined by the dispersion in the moment of inertia, while the width of the narrow component is roughly proportional to the compound damping width $\Gamma_{\text {comp }}$.

For rare-earth nuclei, careful experimental investigations $[137,138,88,139]$ of the $\gamma-\gamma$ energy correlations, combined with simulations of the decay cascades, provide an overall confirmation of compound damping, rotational damping and motional narrowing. Still, it is mainly in simulations that motional narrowing displays the schematic and rather drastic behaviour expressed in Eqs. 27 and 28, whereas in experiments it is more subtle and folded with the entry distribution. 
Extrapolating these results to the SD shape, axis ratio $2: 1$, the most important change is the decreasing dispersion of rotational frequency among the bands, which should lead to a diminishing of the rotational damping width. In the limit of extremely small dispersion, the bands should be able to mix, but the mixed wavefunction does not change from one angular momentum to the next, and this scenario is called ergodic bands [96]. The intrinsic structure of the states is chaotic, with random mixture of many basis band states, while the rotational motion is ordered.

Another characteristic of the second well is the low level density, which may give rise to a larger number of unmixed discrete bands.

Realistic calculations by M. Matsuo, quoted in Sec. 4 of the present review, confirms these overall expectations for rotation in the second well. Concerning the possibility of ergodic bands, it is concluded that the conditions are not strictly met, but rather a more mild condition, corresponding to the precursor of ergodic bands. For the special case of ${ }^{194} \mathrm{Hg}$, this condition may be fulfilled, as indicated by the very narrow ridges in the $\gamma-\gamma$ energy correlation spectrum [70].

\subsection{Probing excited normally-deformed states}

At decay out, in itself, the coupling of the specific SD state to ND states provides a probe of the latter. Especially, for the mass 190 region, the shape of the decay out spectrum in principle could tell about the level density and the strength function with a precisely defined excitation energy and angular momentum. Calculations with standard Fermi-gas level densities yielded a too smooth spectrum compared to experiment, whereas calculations including pairing in the levels structure provided a bumpy spectrum, much better in accordance with experiment [95], as illustrated on Fig. 43. The spectrum at high energy should be dominated by first-step transitions to low lying states, and at low energy it should be dominated by second- or third step transitions to low energy. An experiment applying the fluctuation analysis [94] confirmed this picture.

The SD band state decays out by admixture of a small component of a ND state into its wavefunction. The decay-out spectrum yields information about the nature of this state. For example, for coupling to a doorway state, as displayed in Fig. 18 leads to the kind of transitions displayed in Fig. 20. On the other hand, if the coupling is with compound-states, the decay is statistical in nature. A special information on the states at the energy and angular momentum at decay out is provided by the distribution of transitions strengths of primary transitions in the SD decay out. Only the stronger transitions stand out, as illustrated on Fig. 39 of the present review. If the state is a truly compound state, transition strengths to lower lying states should follow a Porter-Thomas distribution. A thorough investigation of $\chi^{2}$ distributions by means of the maximum likelihood method [140] has confirmed this picture, albeit with certain reservations concerning $(i)$ the intense decay-out transitions, which are quite unlikely, (ii) the limited sample size. It was concluded that for the nucleus ${ }^{194} \mathrm{Hg}$ the states at about 4 $\mathrm{MeV}$ above yrast and at around 12 units of angular momentum, behave as compound states.

\section{Superdeformed gamma flow}

Following its formation in fusion-evaporation reactions, as discussed in Sec. 1.4, the electromagnetic decay of a SD nucleus gives rise to characteristic $\gamma$-ray cascades. First come cascades in the SD well, which cool the nucleus down to the SD yrast line and then along the yrast or near yrast SD bands. Second come the decay-out cascades, which cool the nucleus down again, but this time to the ND yrast line. A schematic picture of such a composite decay flow is given in Fig. 6. This section will be devoted to the study of these two types of cascades and will focuss on what can be learnt from them. Emphasis will be given to Monte Carlo simulations of the $\gamma$-decay flow, which are powerfull tools for a detailed investigation of the average properties of excited superdeformed systems, from their formation, all the 
way down to the ND yrast states.

\subsection{From the entry point to the superdeformed yrast line}

The $\gamma$-decay flow of a SD nucleus can be studied in details by Monte Carlo simulation calculations. A first approach was introduced by Schiffer and Herskind at the Niels Bohr Institute [113] and, independently, by Lauritsen et al. at Argonne National Laboratory with the code KL_SD [141, 59] - the latter being an extension of Refs. $[49,48,142]$ which only described $\gamma$ cascades in the ND well. In both cases the calculations for the SD $\gamma$-decay flow are based on parametrizations of the physical quantities governing the thermal rotation and the tunnelling within wells: level density, gamma strengths, moment of inertia, alignments, damping width, potential barriers etc.. Alternatively, a microscopic treatment can be followed: this is the case of the Monte Carlo code MONTESTELLA, originally developed to study $\gamma$ cascades in normal deformed nuclei, after neutron emission [143, 138, 88], and later extended to describe the $\gamma$-decay flow in both ND and SD wells [73, 75, 93], implementing the model of Schiffer and Herskind [113]. The following sections will describe the main ingredients entering the simulation codes for SD nuclei (see Sec. 4.1.1), and the properties of the two decay modes (E1) and (E2) which are consider in competition, along the SD $\gamma$-decay cascades (see Sec. 4.1.2 and 4.1.3, respectively).

\subsubsection{Nature of excited superdeformed states and simulation of the $\gamma$ flow}

In all Monte Carlo simulation of the $\gamma$ flow, the calculations start from the entry distribution for the $\gamma$-decay cascades (see Sec. 2.2) assigning entry states as SD or ND, with probabilities proportional to their level densities. In the simulation, the feeding and decay of the SD bands are based on the competition between statistical E1 dipole transitions (cooling the nucleus without a significant change of its angular momentum, being $\Delta I=0,1,-1$ ), collective rotational E2 transitions (mostly taking place at constant temperature, with $\Delta I=2$ ) and the tunnelling across the barrier separating the ND and SD configurations (cf Sec. 3) [95, 119].

The peculiarity of the MONTESTELLA model, extensively employed to investigate the decay flow of the SD nuclei ${ }^{151} \mathrm{~Tb}$ and ${ }^{196} \mathrm{~Pb}[73,75,93]$, is the use, in the SD well, of energy levels, E2 transition probabilities and potential barriers microscopically calculated by the cranked shell model of Yoshida et al. [144, 145, 97]. This is crucial for many aspects of the analysis, since the use of microscopically calculated quantities i) reduces the number of parameters used by the simulation code, ii) makes it possible to perform a meaningful fluctuation analysis of the simulated cascades, iii) allows for a selective study of decay paths feeding specific rotational bands, the latter being strongly influenced by the properties of the decay flow. A schematic illustration of the $\gamma$ flow is given on the left of Fig. 23, pointing to the main ingredients of the model, while the right hand side of the figure shows the calculated average heat energy (U, cf Eq. 22) of the decay flow in the SD well, as a function of spin, in the case of the ${ }^{151} \mathrm{~Tb}$ nucleus, here taken as a reference in the discussion. In the simulation, the $\gamma$ decay starts from a spin and excitation energy entry distribution calculated by fusion-evaporation models [146, 147], giving for ${ }^{151} \mathrm{~Tb}$ an average excitation energy of the order of $8.3 \mathrm{MeV}( \pm 2.5 \mathrm{MeV})$ and the spin cross section shown in the top panel of Fig. 23 (right). Alternatively, the entry distribution can be measured, as discussed in Sec. 2.2 and shown in Fig. 14 [59], further reducing the number of parameters entering the simulation.

The energy levels and E2 transition probabilities associated with the SD configurations used in the MONTESTELLA code are calculated microscopically for each of the four parity and signature, $(\pi, \alpha)$, configurations over the entire region of populated spins and with excitation energies up to $\mathrm{U} \approx 4-5 \mathrm{MeV}$ above yrast line (depending on the nuclear system), i.e. into the excitation energy region where discrete spectroscopy looses its meaning $[144,145]$. The calculations combine the cranked Nilsson mean field and a residual two-body force: np-nh configurations, defining unperturbed rotational bands, are first 

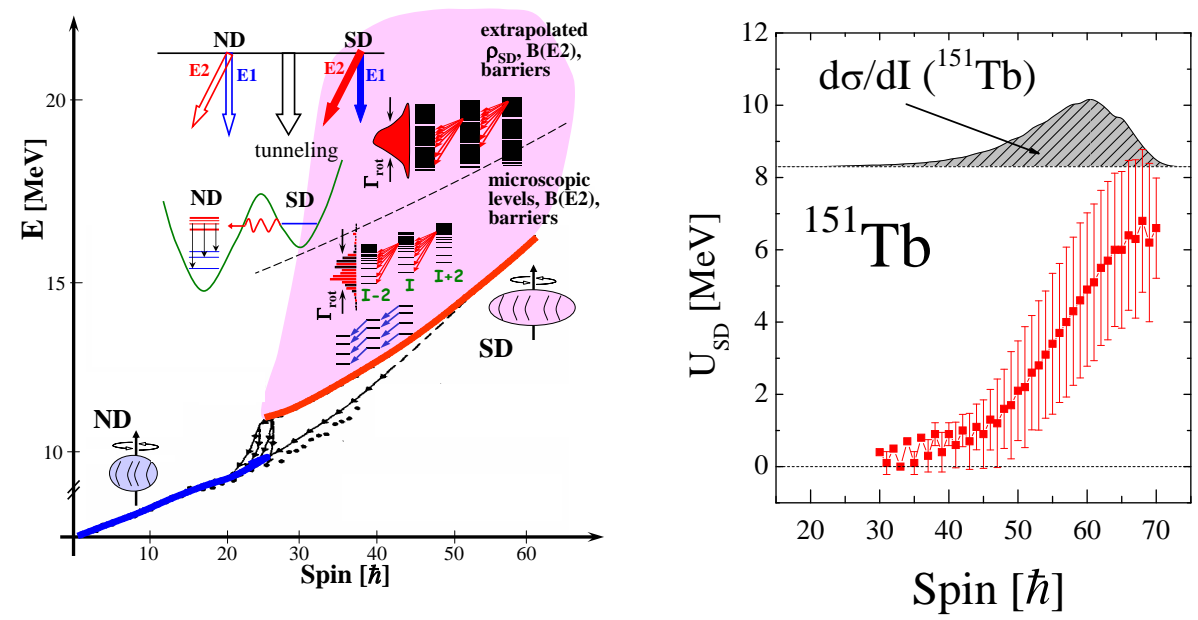

Figure 23: (Color online) Left: Schematic illustration of the decay scheme used by the Monte Carlo simulation MONTESTELLA, describing the $\gamma$ decay of a warm rotating nucleus within two potentials (i.e. the ND and SD wells). The shaded area indicates the spin-excitation energy region where the $\gamma$ decay flow assumes a SD character [93]. The excitation energy region where microscopically calculated levels, B(E2) strength and barriers are used is delimited by the dashed line. Right: Calculated average heat energy of the decay flow in the SD well as a function of spin for simulated $\gamma$-cascades starting from the differential spin cross sections given in the same panels by shaded areas.

constructed and a residual two-body interaction, delta type, in then diagonalized. The corresponding E2 transition probabilities connecting the mixed states are then calculated, assuming that the rotational decays remove 2 units of spin $(\Delta I=2 \hbar)$, as supposed by the MONTESTELLA simulation.

Figure 24 shows the calculated energy spectra of the lowest discrete bands for each $(\pi, \alpha)$ configuration of ${ }^{151} \mathrm{~Tb}$, in reasonable agreement with the yrast line and first excited bands experimentally observed [93]. The calculated bands (less than 100 in ${ }^{151} \mathrm{~Tb}$ ) are obtained by selecting the energy levels decaying by strong E2 transitions within a rotational band for at least two steps, namely decaying at each step to less than two states. This can be defined by the branching number

$$
n_{\mathrm{b}}=1 / \sum_{\beta} w_{\alpha \rightarrow \beta}^{2}
$$

associated with the E2 strength for decays from a level $\alpha, w_{\alpha \rightarrow \beta}$ being the normalized E2 strength from a level $\alpha$ at I to a level $\beta$ at I-2. This quantity measures effectively the number of E2 decay branches and can be used to define the onset of rotational damping [132] by the condition that the E2 decays have more than two branches $\left(n_{\mathrm{b}}>2\right)$. In ${ }^{151} \mathrm{~Tb}$ this condition is satisfied at heat energy $U \approx 3 \mathrm{MeV}$, as shown in the right panel of Fig. 25, above which a progressive fragmentation of the E2 strength is observed. The FWHM of such B(E2)'s distributions is named rotational damping width, $\Gamma_{\text {rot }}$. This damping process, discussed in Sec. 3.6, is a direct consequence of the different properties of the mean field configurations: they react differently to the rotation and are mixed by the residual interaction into the compound nucleus states discussed above [132]. Figure 25, left column, gives examples of the fragmentation of the E2 distributions, as a function of increasing energy $U$, for ${ }^{151} \mathrm{~Tb}$ at $\mathrm{I}^{\pi}=50.5^{+}$.

The cranking calculations of Refs. $[144,145]$ give also the possibility to investigate in details the composition of the microscopic levels entering the simulation. Figure 26 gives the mixing number $n_{m i x}$ for states in ${ }^{151} \mathrm{~Tb}$ (left) and ${ }^{196} \mathrm{~Pb}$ (right), calculated at $I^{\pi}=50.5^{+}$and $I^{\pi}=30^{+}$, respectively. $n_{m i x}$ 

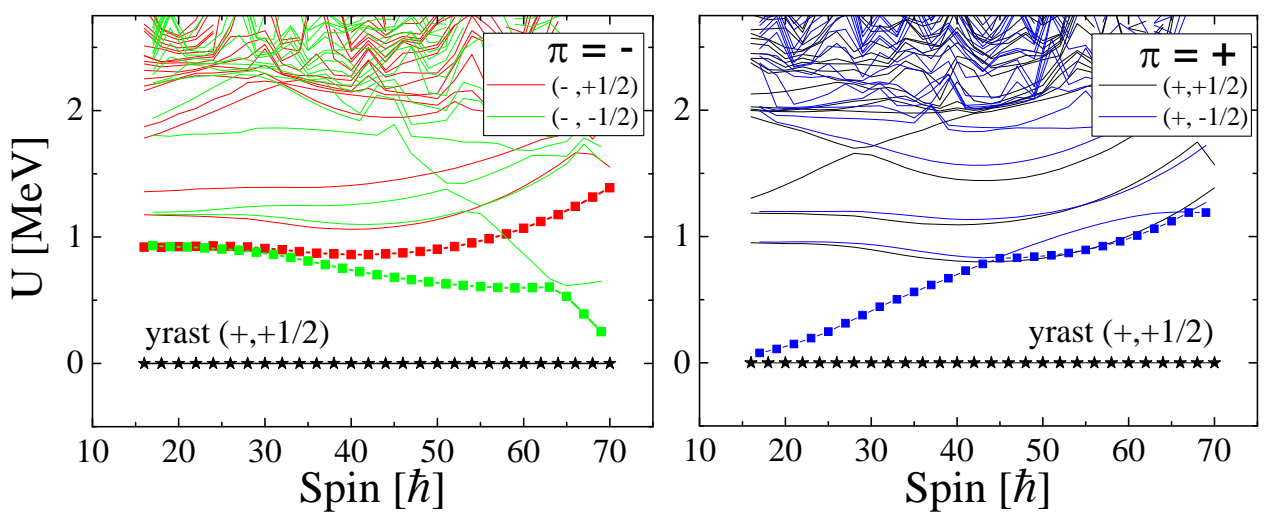

Figure 24: (Color online) Excitation energy spectrum of the discrete SD rotational bands for positive (right) and negative (left) parity states of ${ }^{151} \mathrm{~Tb}$, calculated with the cranked shell model of Ref. [144]. For each $(\pi, \alpha)$ configuration the lowest bands are shown up to $2.75 \mathrm{MeV}$ of excitation energy above the absolute $(+,+1 / 2)$ SD yrast line (shown by stars). Square symbols represent the SD yrast line of the other $(\pi, \alpha)$ configurations [93].

effectively measures the number of unperturbed states mixed by the residual interaction into a given energy compound nucleus state, therefore it increases strongly with the heat energy $U$, since the level density increases exponentially with $U$ (cf Eq. 20). In the figure, the full symbols correspond to states for which the decay occurs within the same band for at least two consecutive transitions, namely they correspond to the discrete rotational bands defined by the condition $n_{\mathrm{b}}<2$ (cf Fig. 25 (right)). As discussed in Refs. [75, 93], in the case of ${ }^{151} \mathrm{~Tb}$ discrete SD bands are composed by a few dominant components, as typically occur in ND nuclei [134]. On the contrary, in the SD nucleus ${ }^{196} \mathrm{~Pb}$ one finds states which form discrete bands and at the same time show much larger mixing numbers (up to 30), especially at low spins. This indicates rather complex wave functions, a special feature also observed in other nuclei of mass $\mathrm{A} \approx 190$, such as ${ }^{194} \mathrm{Hg}$ [70]. In that case, a very large number of bands (up to $\approx 150$ ) is observed at the highest spins. Such unusual feature has been interpreted as a manifestation of an ergodic regime, namely of an intermediate situation between order and chaos, where rotational coherence is preserved, although the wave function contains many mean-field configurations [96], as already mentioned in Sec. 3 and will be later discussed in Sec. 4.1.3.

Concerning the E1 cooling mechanism in both ND and SD well, the E1 decay probability for a given $\Delta I$ value $(\Delta I=0, \pm 1)$ is defined as

$$
T_{\Delta I}\left(E 1, U_{i}\right)=h_{\mathrm{E} 1} \sum_{j=1,2,3} \int_{0}^{U_{i}} T_{j}\left(U_{i}, U_{f}\right) d U_{f}
$$

where

$$
T_{j}\left(U_{i}, U_{f}\right)=E_{\gamma}^{3} f_{\mathrm{GDR}, \mathrm{j}}\left(E_{\gamma}\right) \frac{\rho\left(U_{f}\right)}{\rho\left(U_{i}\right)}
$$

The level densities $\rho(U)$, for initial $i$ (final $f$ ) states at the initial (final) excitation energy $U_{i}\left(U_{f}\right)$ are given by the usual Fermi gas function (cf Eq. 20 [148]), with a level density parameter, in the SD well, obtained by fitting the microscopic results of the cranking calculations discussed above [144, 145].

In the previous expression $f_{\mathrm{GDR}, \mathrm{j}}$ is the strength function associated with the giant dipole resonance (GDR) along the vibrational axis $j$ (normalized so that the total GDR strength exhausts the E1 sum rule). The functions $f_{\mathrm{GDR}, \mathrm{j}}$ used in the ND (SD) well are assumed to be Lorentzians, and correspond to 


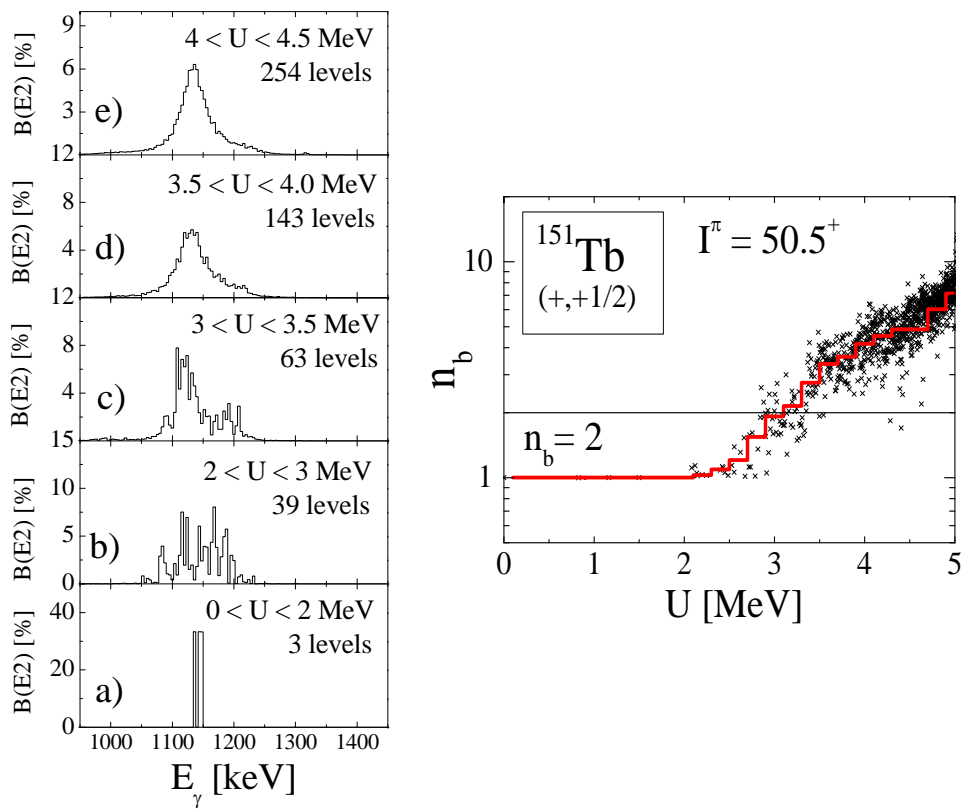

Figure 25: (Color online) Left: B(E2) strength functions microscopically calculated by the model of Ref. [144], at $\mathrm{I}^{+}=50.5^{+}$in ${ }^{151} \mathrm{~Tb}$, averaged over the number of levels within the interval in excitation energy $U$ given in each panel. Right: E2 branching number $n_{b}$ as a function of excitation energy $U$ for the states at $\mathrm{I}^{+}=50.5^{+}$of ${ }^{151} \mathrm{~Tb}$. The histogram shows $\mathrm{n}_{b}$ averaged over energy bins of $200 \mathrm{keV}$. The horizontal line gives the $\mathrm{n}_{b}=2$ level, corresponding to the onset of rotational damping.

a nucleus with deformation parameters consistent with the ND (SD) configuration. As shown in Fig. 27 in the case of ${ }^{151} \mathrm{~Tb}$, in the ND well the GDR is described by a single Lorentzian, with centroid and width $E_{\mathrm{GDR}}=15.8 \mathrm{MeV}$ and $\Gamma_{\mathrm{GDR}}=4 \mathrm{MeV}[149]$. In the SD well, instead, the two Lorentzians associated with the two shorter axes have centroids $E_{\mathrm{GDR}}=18.7 \mathrm{MeV}$ and width $\Gamma_{\mathrm{GDR}}=5.7 \mathrm{MeV}$, while that associated with the longer axis has $E_{\mathrm{GDR}}=8 \mathrm{MeV}$ and $\Gamma_{\mathrm{GDR}}=3 \mathrm{MeV}$, according to the calculations of Ref. [150]. As compared to the ND strength, a substantial shift of the E1 strength is observed in the SD case at energies $E_{\gamma}$ around the particle binding $(\approx 8-10 \mathrm{MeV})$ : this is considered to be relevant for the population of the SD states, as discussed in Sec. 4.1.2. In the region of the microscopic levels, the integral in Eq. (30) is replaced by a summation.

In Eq. 30 a hindrance factor $h_{\mathrm{E} 1}$ is introduced and adjusted, in the SD well, to reproduce the experimental intensity of the SD yrast line $\left(\approx 2 \%\right.$ in $\left.{ }^{151} \mathrm{~Tb}\right)$. This is in agreement with previous work on ND nuclei, showing that in order to reproduce the average experimental intensity of low-lying bands, a reduction of the $\mathrm{T}(\mathrm{E} 1)$ for each $\Delta \mathrm{I}=0,1$ and -1 decay component is needed (by a factor of $\approx 50$ $[143,82])$, as follows from systematic experimental studies of $\gamma$-ray transition probabilities for different values of $\Delta K$ of the $K$ quantum number [151]. In addition, as shown in the inset of Fig. 27, the low energy part of the E1 strength function is further modified, in the SD well, by adding an extra component $f_{\mathrm{OCT}}$ of Laurentzian shape, with centroid and width $E_{\mathrm{OCT}}=1.5 \mathrm{MeV}, \Gamma_{\mathrm{OCT}}=1.0 \mathrm{MeV}$ and intensity scaled by a factor $h_{\mathrm{OCT}}$. This turns out to be crucial for a proper reproduction of the intensities of the $1^{\text {st }}$ ridge and $\mathrm{E} 2 \mathrm{QC}$ in coincidence with the SD yrast line, which are observables very sensitive to the delicate balance between E2 and E1 transition probabilities at low excitation energies, where specific nuclear structure effect become important. In the case of $A=150$ and 190 mass regions, strongly enhanced E1 transitions were indeed observed, linking excited SD bands to the yrast line $[152,153,17,154]$. These excited bands have been interpreted as octupole phonon excitations. In the Monte Carlo calculations, the intensity of the extra octupole component is adjusted so that the total E1 strength $f_{\mathrm{E} 1}=f_{\mathrm{OCT}}+f_{\mathrm{GDR}}$ is enhanced by about one order of magnitude around 1-2 MeV, consistently 


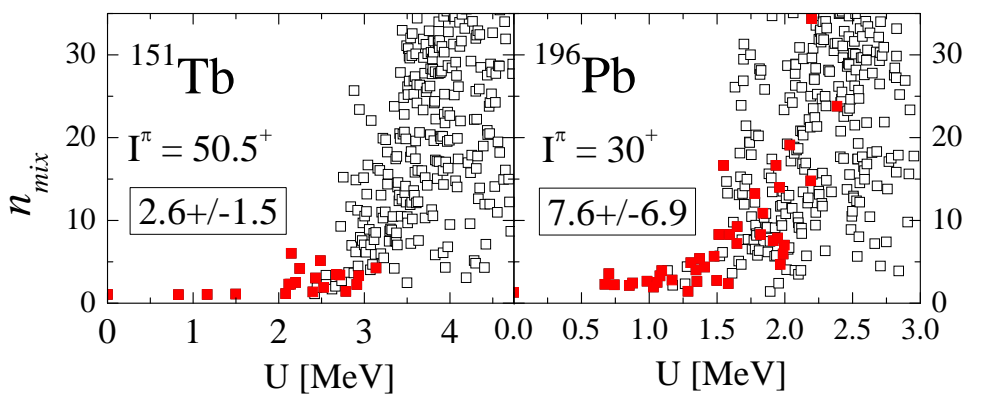

Figure 26: (Color online) Number $n_{m i x}$ of admixed stated for $I^{\pi}=50.5^{+}$in ${ }^{151} \mathrm{~Tb}$ (left) and $I^{\pi}=30^{+}$ in ${ }^{196} \mathrm{~Pb}$ (right), as a function of heat energy U, calculated by the model of Ref. [144]. The full symbols correspond to states for which the decay occurs mainly within the same band for two consecutive transitions (i.e., $\mathrm{n}_{b}<2$ ), with an average mixing number given in the legend (adapted from Ref. [93]).

with the experimental evidence, resulting in hindrance factors $h_{\mathrm{E} 1}$ and $h_{\mathrm{OCT}}$ of the order of 0.03 in ${ }^{151} \mathrm{~Tb}[75,93]$.

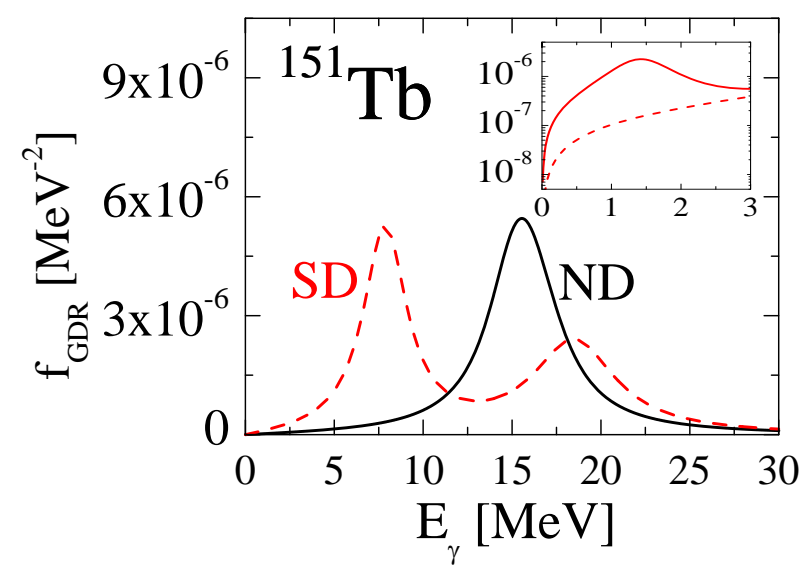

Figure 27: (Color online) The E1 decay strength $f_{\text {GDR }}$ used in the Monte Carlo simulation code in the case of ${ }^{151} \mathrm{~Tb}$. The full thick line corresponds to the ND configuration, while the dashed line gives the E1 strength for the SD case. The inset shows the low energy part of the E1 strength function, including or not the extra octupole component (solid and dashed lines, respectively) discussed in connection with the population of SD discrete excited bands (see text for details).

Finally, the tunnelling across the potential barrier between the ND and SD wells is described, in MONTESTELLA, following the original decay model of Schiffer and Herskind [113], while the description of Bjornholm and Lynn [6] has been implemented in the Monte Carlo approach of Lauritsen et al. [141]. In both cases, the width $\Gamma_{S D \rightarrow N D}$ for tunnelling across the potential barrier between the SD and ND well, which is crucial for a proper description of the $\gamma$ decay of a SD nucleus, is evaluated semiclassically, as described by Eq. 18 in Sec. 3.2. In the MONTESTELLA approach the tunnelling calculations are based on a microscopic treatment of the action integral along the tunnelling path connecting the SD and ND wells in the $(\epsilon, \gamma)$ plane (see Eqs. 12-14), being based on the Nilsson-Strutinsky potential energy surface $V$, including pairing correlations, and on the mass parameter $m_{0}^{(h o p)}$, which is a function of the deformation parameters (cfr. Figs. 16 and 17). Both the potential surface and the mass depend on spin [97].

Fig. 28 shows a comparison between experimental (left) and simulated (right) one-dimensional (1D) 
and projections of two-dimensional (2D) $\gamma$ spectra of ${ }^{151} \mathrm{~Tb}$. Panels a), c) and e) give examples of cuts perpendicular to the main diagonal of the $2 \mathrm{D} \gamma$-coincidence spectra, for the wide spin intervals $\approx 48-56$ $\hbar$. Panel a) refer to total $\gamma-\gamma$ matrices, c) to the rotational planes and e) to the SD-gated spectra. In all cases, ridge structures are clearly visible, with a spacing between the two most inner ridges proportional to $\Delta E_{\gamma}=4 \hbar^{2} / \Im^{(2)} \approx 50 \mathrm{keV}$, where $\Im^{(2)}$ is the moment of inertia of the SD yrast band. The intensities of the known ND and SD discrete peaks, subtracted from the Total and SD-gated matrices making use of the RADWARE package [155], before performing any further analysis of the data, are indicated by dark areas. In the case of the SD states, the only significant contribution comes from the SD yrast transitions. In the case of the rotational planes spectra the cuts have been instead obtained by projecting the 2D-spectra in between the SD yrast peaks, therefore resulting in event distributions free from strong contaminants from SD yrast lines. In this case, the most inner ridge corresponds to the second ridge in the Total and SD-gated matrices, and its spacing is therefore twice as large. The top panel g) of both figures gives the 1D QC spectra double gated by the SD-yrast band, with the energy scale on the top axis. In both experimental and simulated spectra the SD yrast band is clearly visible and the broad bump above $\approx 1100 \mathrm{keV}$ is made mostly of unresolved E2 transitions forming the E2 QC. In the data, the shaded area at low energies (i.e. below $\approx 1100 \mathrm{keV}$ ) may be attributed to possible M1 contributions (not included in the simulation). In fact, it is known, from the experimental analysis of both $\mathrm{A} \approx 150$ and 190 mass regions, that the $\gamma$ rays at the end of the cascades feeding the SD yrast band may have large negative $A_{2}$ values (e.g., see $[59,156,15,157,158,65,159]$ ). This can only be explained if they are of mixed M1/E2 nature. As pointed out in the outlook section, an important development of present simulation calculations would be the implementation of M1 and M1/E2 decay modes in order to properly describe the last feeding steps into the SD (and ND) yrast line. This is a rather delicate issue, since at low excitation energies selections rules govern the $\gamma$ decay very strongly.

It is worth noticing that the Monte Carlo simulation MONTESTELLA, here discussed, as well as the independent KL_SD model of Lauritsen et al. [141, 59], are able to reproduce remarkably well the line shape of the experimental $\gamma$ spectra and the relative intensities of the different spectral components. Moreover, other sensitive quantities, such as the number of discrete unresolved bands, can also be computed by the MONTESTELLA code (see later discussion in Sec. 4.1.3), giving confidence in the theoretical calculations and in the general good description of the dynamics of the decay flow in the SD well. As a consequence, simulation calculations have been extensively used to shed lights on specific properties of the $\gamma$-flow, as discussed in the two following sections in connection with the E1 and E2 components of the decay cascades.

\subsubsection{E1 cooling}

It is well established that the relative intensity of the SD band at the highest spins is much larger than the observed intensities of ND structures, as shown in Fig. 29 in the case of ${ }^{152}$ Dy [113]. Such a feature is common to all SD yrast bands experimentally found in different regions of mass.

Besides the fact that the SD yrast line is always energetically lower than the ND yrast line above a certain spin (e.g., $54 \hbar$ in the case of ${ }^{152} \mathrm{Dy}$ and $30 \hbar$ in the case of ${ }^{194} \mathrm{~Pb}$ ), a model has been proposed to describe the enhanced feeding of the SD yrast band by E1 cooling, at the highest spins, by more than one order of magnitude above standard cooling rates $[160,113]$. Two different effects are the key ingredients of the model: i) the E1 enhancement by the low lying GDR component in the SD well and ii) the enhancement of the E1 strength by the lower density of SD states (cf Sec.3.5).

Several experimental attempts have been made to prove the proposed E1 cooling mechanism and up to now only indirect evidences have been obtained, leaving such an important problem still open. In fact, the most compelling evidence would be based on a direct observation of a low lying GDR component, 


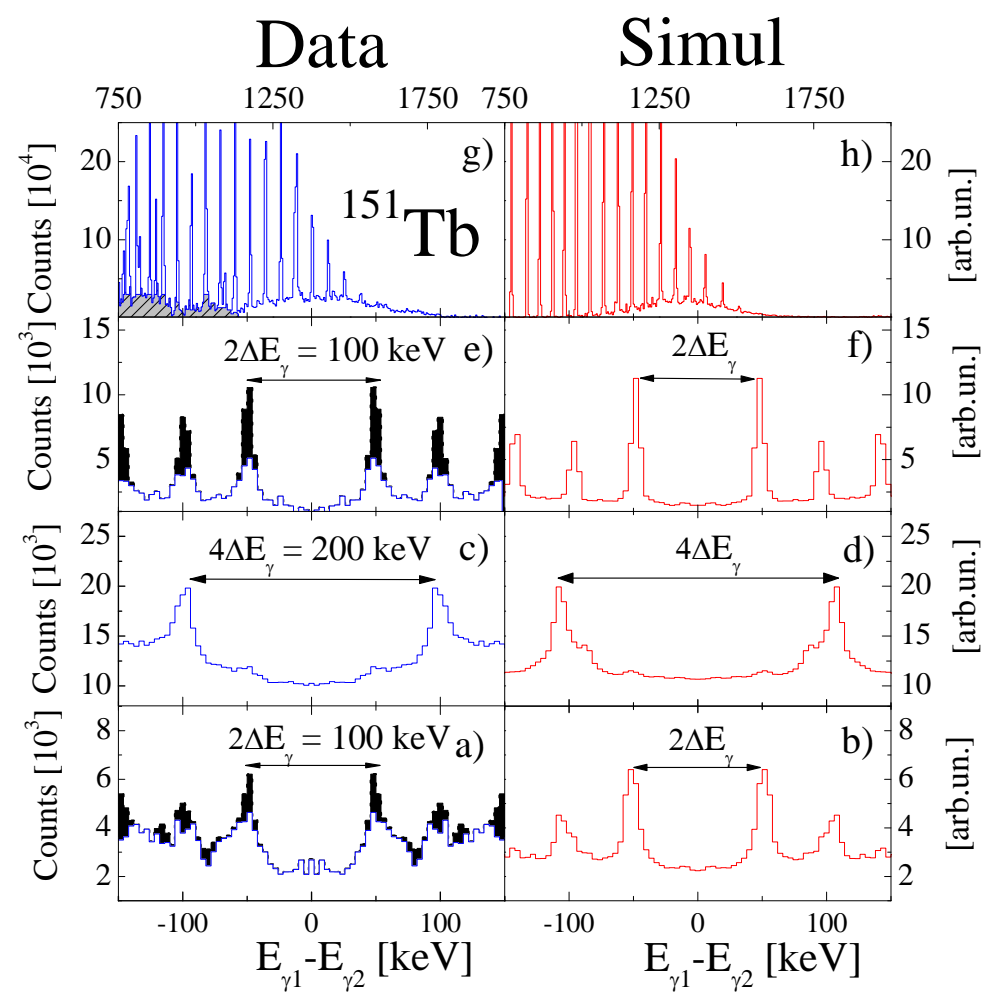

Figure 28: (Color online) Experimental and simulated spectra of ${ }^{151} \mathrm{~Tb}$. Panels (a)-(f) show projections perpendicular to the main diagonal of the total $\gamma-\gamma$ coincidence matrices $[(\mathrm{a}),(\mathrm{b})]$, of the rotational planes $[(\mathrm{c}),(\mathrm{d})]$, and of the SD-gated $\gamma-\gamma$ matrix $[(\mathrm{e}),(\mathrm{f})]$, over the spin interval $48-56 \hbar$. The dark areas in panels (a) and (e) correspond to the intensity of known ND and SD discrete peaks subtracted from the spectra by the RADWARE package [155]. Panels (g) and (h) show 1D spectra gated by the SD yrast band; the shaded area in the experimental spectrum corresponds to the region where contaminants from M1s are expected, as observed in neighbouring nuclei (see text for details).

when gating on the SD yrast band. In the work of Ref. [161] an attempt was made with EUROBALL (EB) coupled to the 8 large volume $\mathrm{BaF}_{2}$ detectors of the HECTOR array (for the measurement of high-energy $\gamma$ rays) [162]. The studied case, the ${ }^{143} \mathrm{Eu}$ nucleus, is characterised by a rather strongly populated yrast band (at the level of 3\%) in addition to peculiar features which may favour this type of investigation. At low spins, due to the coexistence of both spherical (ND) and triaxially deformed (TD) shapes this nucleus has a very complex and irregular level scheme, while it becomes SD at high spins. Both the SD yrast band and the excited SD states of the E2 continuum follow decay routes leading to low spin states of the spherical shape only (ND) [163]. As shown in the right panel of Fig. 30 , the high energy $\gamma$-ray spectrum double gated by the lines of the SD yrast band (filled circles) gives indication of an excess yield in the region around $10 \mathrm{MeV}$ (in spite of the low statistics), compared to the spectrum gated by low spin transitions of the triaxial shape, not populated by SD states. A similar type of excess is also observed in the high-energy spectrum gated by spherical transitions (left panel of Fig. 30), populated by SD configurations. This observation may indicate a possible SD nature of the observed excess and clearly demonstrates the difficulties of such a type of investigation, which is strongly limited by lack of statistics. It is important to mention that a first attempt to this type of analysis was made in the late 80 's, by P. Taras et al. [164] in the case of ${ }^{149} \mathrm{Gd}$, by employing the $8 \pi$ spectrometer [164]. 


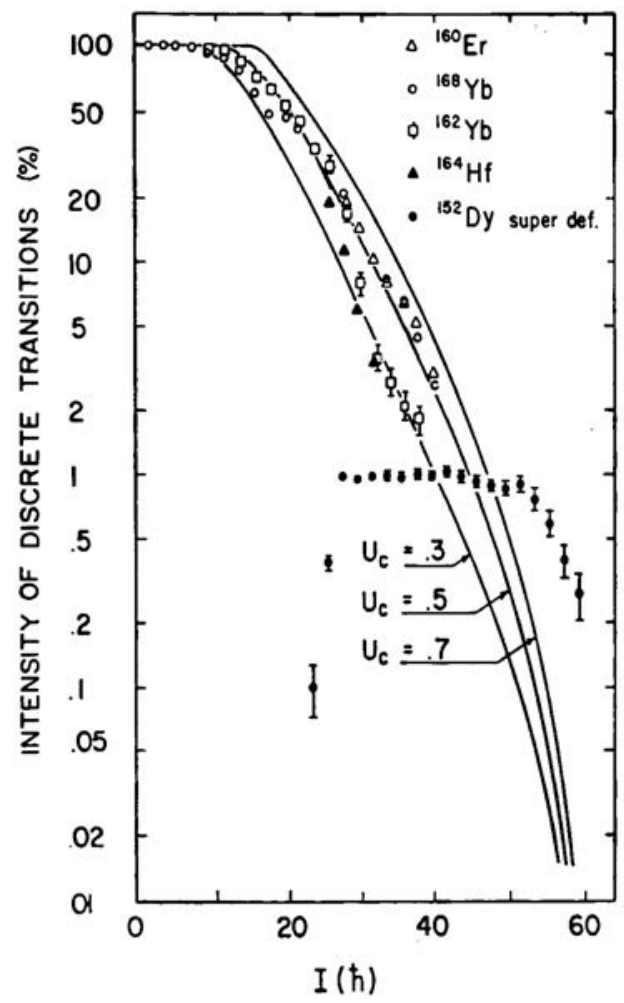

Figure 29: Intensities of the known discrete lines (at a given spin) in the well deformed nuclei ${ }^{160} \mathrm{Er}$, ${ }^{180} \mathrm{Yb}$ and ${ }^{164} \mathrm{Hf}$ and of the SD band of ${ }^{151} \mathrm{Dy}$. The solid lines show the calculated intensity for the case of ${ }^{168} \mathrm{Yb}$, as follows from the model of Ref. [113] which collects in the yrast band the E1 decay flux below an energy $U_{c}$ (given in $\mathrm{MeV}$ in the figure). A similar approach is also used in the KL_SD model of Lauritsen et al., [59].

The Monte Carlo simulations of the $\gamma$-decay flow discussed in Sec. 4.1.1, being based on the E1 cooling model of Ref. [113], also suggested more indirect experimental techniques to prove the proposed mechanism. They consist in gating on high energy transitions in the region of the expected low lying component of the GDR built on the SD system and in measuring any possible enhancement of intensity in the SD structures. This method was applied, in the case of ${ }^{143} \mathrm{Eu}$, to the yrast band, the discrete excited SD bands (forming the QC ridges) and the strongly interacting bands (giving rise to the major fraction of the QC E2 distribution in one dimensional spectra). As shown in Fig. 31, the population of the SD states is found to be enhanced by a factor of $\approx 1.6$, when a coincidence with a $\gamma$-ray with energy $>6 \mathrm{MeV}$ is requested. This is in agreement with the increase of the line shape of the Giant Dipole Resonance built on the SD configuration, thus giving support to the E1 cooling model of Herskind et al. [160].

Additional experimental indication of a highly fragmented GDR strength function, with a low energy component associated to the presence of a large deformation and Coriolis effects, has been obtained in lighter systems, around mass $\mathrm{A} \approx 40$ [166, 167, 168, 169]. In particular, in the case of ${ }^{46} \mathrm{Ti}$, the GDR lineshape shows evidence for a Jacobi shape transition, namely an abrupt change of nuclear shape from an oblate ellipsoid, rotating non-collectively around its symmetry axis, to an elongated prolate or triaxial shape, rotating collectively around the shortest axis, at angular momenta close to the fission limit [170, 171]. Figure 32 (left) shows the experimental GDR strength function of the compound nucleus ${ }^{46} \mathrm{Ti}$, populated in the ${ }^{18} \mathrm{O}+{ }^{28} \mathrm{Si}$ reaction at $105 \mathrm{MeV}$ bombarding energy, measured with the EB IV Ge-array coupled to the HECTOR scintillator array and the charged particle detector 

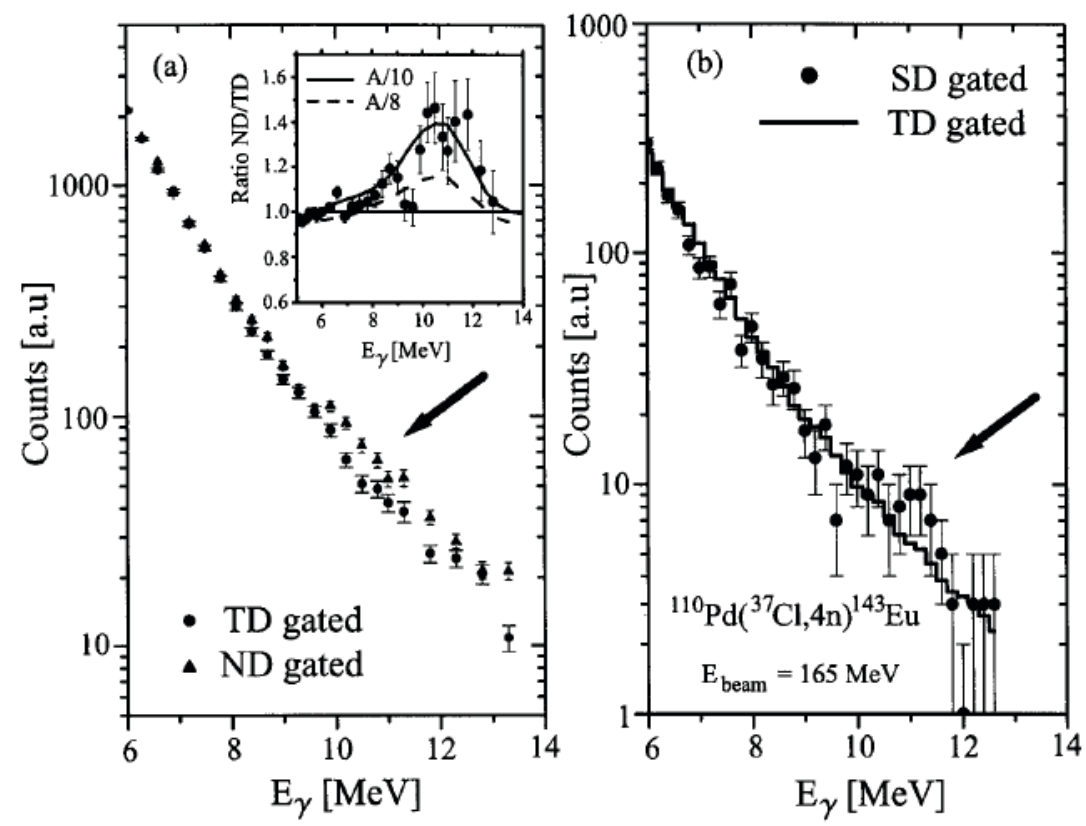

Figure 30: Left panel: High-energy spectra of ${ }^{143} \mathrm{Eu}$ constructed by selecting two transitions depopulating the spherical (ND) structure (triangles) or the triaxial (TD) configuration (circles). The ratio of the two spectra is shown in the inset, in comparison with calculations from the statistical model of the compund nucleus decay [147], assuming different values for the level density parameter in the SD well (i.e. A/8 and A/10). Right panel: Comparison of the high-energy $\gamma$-ray spectrum double gated by the lines of the SD yrast band (circles) with the spectrum gated by the triaxial configuration (line) [161].

EUCLIDES [172]. Data are compared with theoretical predictions of the GDR lineshape based on the thermal shape fluctuation model, taking also into account the Coriolis splitting effect, which can be observed in light systems where the highest rotational frequency values can be reached. As a result, a pronounced and narrow peak is observed in the GDR strength function, around $10 \mathrm{MeV}$, as expected for a prolate or triaxial fast rotating nucleus [168]. Moreover, this low energy component of the GDR was found to feed preferentially the highly-deformed band of the residual nucleus ${ }^{42} \mathrm{Ca}$, as shown on the right of Fig. 32, similarly to what observed in the case of ${ }^{143} \mathrm{Eu}$, discussed above (cfr. Fig. 31). It is interesting to note that indications of large deformations of the compound nucleus ${ }^{46} \mathrm{Ti}$ were also obtained by an independent measurement of charged particle emission [173].

One can then conclude that the experimental results on both ${ }^{143} \mathrm{Eu}$ and ${ }^{46} \mathrm{Ti}$ give tentative support to the hypothesis of Herskind et al. [160] of a special role played by the low energy component of the GDR in feeding the SD structures, although new measurements are needed for an unambiguous conclusion.

\subsubsection{Rotation, tunnelling and ergodic bands at high temperature}

In this section we concentrate on the analysis of quasi-continuum 1D and 2D $\gamma$-coincidence spectra, focusing in particular on the unresolved spectral components associated to the warm rotational motion: the ridge structures, populated by discrete excited rotational bands, and the continuum distributions of E2 character (in one dimension), collecting the population of strongly interacting bands from the rotational damping region. Such components can be isolated in the spectra as described in Sec. 2.1 and illustrated in Fig. 28, in the case of ${ }^{151} \mathrm{~Tb}$. 


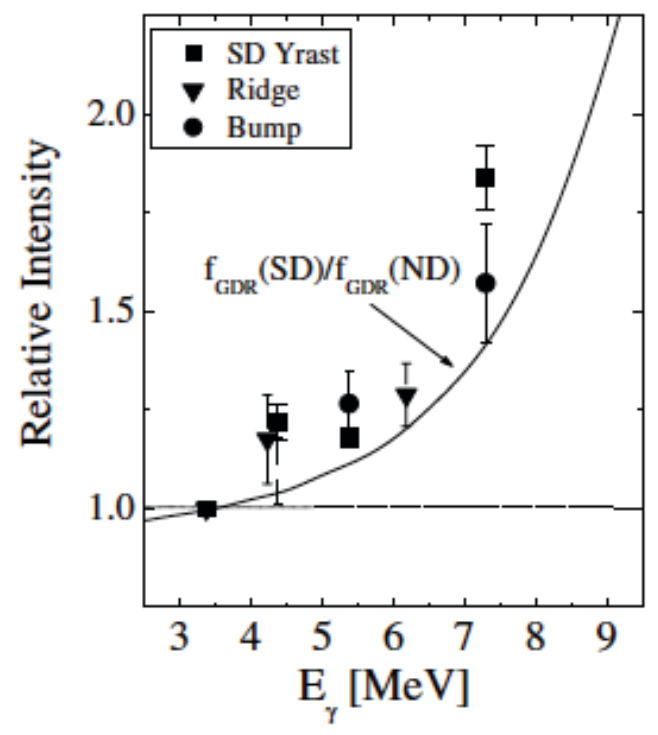

Figure 31: Intensity of the SD yrast band (squares), ridges (triangles) and E2 QC distribution (circles, called 'bump'), of ${ }^{143} \mathrm{Eu}$, as a function of the high-energy gating transition (normalized at $3 \mathrm{MeV}$ ). The full curve gives the ratio between the SD and the spherical GDR strength functions. It represents a lower limit for the feeding of the SD nucleus by E1-decay from the GDR (Adapted from Ref. [165]).

The experimental data can be analysed in terms of spectral shapes, intensities and count fluctuations of the event distributions. A parallel analysis can be performed on simulated spectra, if microscopic levels are included in the model, as in the case of the Monte Carlo code MONTESTELLA [75, 93]. The comparison between theory and experiment will focus exclusively on 1- and 2-dimensional spectral shapes and intensities, if simulation calculations are based on parametrization of the physical quantities: this is the case of the KL_SD simulation model [141, 59].

Figure 33 gives an example of a complete spectroscopy analysis of the ridge structures in the SD nucleus ${ }^{151} \mathrm{~Tb}$, both in terms of intensities (left) and count fluctuations (right). In the figure, data and calculations, performed by the MONTESTELLA code, are compared on equal footing, considering the entire ensemble of unresolved discrete SD bands, as follows from the study of the total population of the $1^{\text {st }}$ and $2^{\text {nd }}$ ridge (panels a), b) and c), d), respectively) and by selecting rotational structures feeding into the SD yrast band (panel e),f)) [73]. The analysis is based on the QC spectra shown in Fig. 28. Similar types of analysis were performed, at least partially, in a few SD nuclei of mass $A=130, A=150$ and $\mathrm{A}=190$, contributing to delineate common features and properties of the rotational motion at high temperature, in highly deformed systems.

The relative intensity of the SD ridges of ${ }^{151} \mathrm{~Tb}$ is shown in the left column of Fig. 33, as a function of spin, always referred to the intensity of the SD yrast line. The yrast band collects $\approx 2 \%$ of the population of ${ }^{151} \mathrm{~Tb}$ and is normalized to $100 \%$ in the low spin part of the plateau region (i.e. for $\mathrm{I} \leq 50 \hbar)$. The total intensities of the 1 st and 2 nd ridge (panels a) and b)) are found to be up to 3 times larger than the population of the SD yrast band at the plateau: this indicates the existence of several discrete unresolved SD bands which do not necessarily feed the SD band, but decay to ND states, as also reported in the case of the SD nuclei ${ }^{143} \mathrm{Eu},{ }^{163} \mathrm{Lu}$ and ${ }^{196} \mathrm{~Pb}[92,67,73]$. The more selective analysis of the ridge structure in direct coincidence with the SD yrast band is shown in panel e). Data indicate that at most $40 \%$ of the yrast intensity comes from discrete excited bands, leaving an important contribution to fragmented/damped bands at higher excitation energies, where rotational damping is expected to play a major role. This is confirmed by the analysis of the E2 QC, giving rise to the distribution observed in the 1D spectra shown in Fig. $28 \mathrm{~g}$ ) and $\mathrm{h}$ ), which is only partially exhausted by the ridge intensity [75]. This seems a common feature of other nuclei of mass 150 and 190 , 

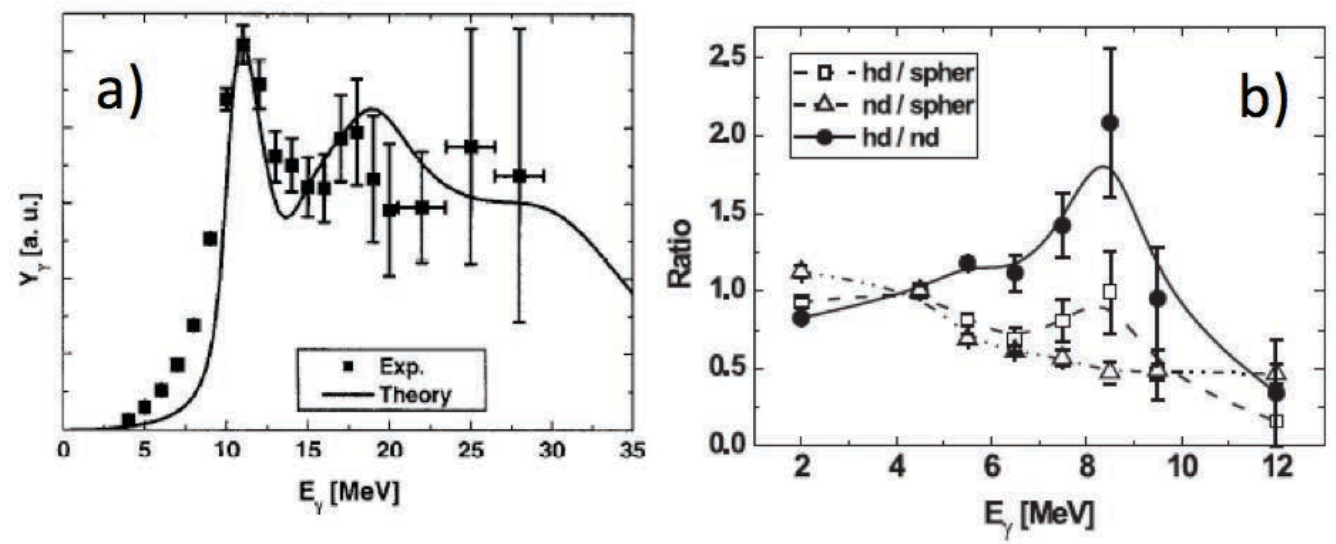

Figure 32: Left: Comparison between experimental data and theoretical predictions for the lineshape of the GDR of ${ }^{46} \mathrm{Ti}$. The calculations are based on the thermal shape fluctuation model, taking also into account the effect of the Coriolis splitting [168]. Right: Intensity ratios between $\gamma$ transitions from the highly-deformed band of ${ }^{42} \mathrm{Ca}$ (denoted as "hd") and normally-deformed ("nd") or spherical ("spher") transitions in the same nucleus, as a function of the $\gamma$-ray energy from the GDR decay. An enhancement in the population of the highly-deformed band is observed in the 8-9 MeV region, which corresponds to the $10 \mathrm{MeV}$ low energy component of the GDR strength function, after taking into account the detector's response [169].

such as ${ }^{152} \mathrm{Dy}$ [59] and ${ }^{196} \mathrm{~Pb}$ [73], where most of the feeding of the SD yrast line comes from damped bands. A notable exception is ${ }^{194} \mathrm{Hg}$, where the ridge intensity accounts for all of the E2 QC (see later discussion and Fig. 34).

By comparing experimental results on spectrum intensities with model predictions, as shown in the right column of Figs. 28, it is found that the MONTESTELLA simulation calculations reproduce rather well the line shape of the $\gamma$ spectra, both concerning the ridges and the total E2 QC in coincidence with the SD yrast band. In addition, they account well for the SD yrast pattern and the total population of the discrete excited bands forming the $1^{\text {st }}$ and $2^{\text {nd }}$ ridge (lines in Figs. 33 a) and c)), with little adjustment of the simulation parameters. A significant increase of the E1 strength function, in the 1-2 MeV region, is instead needed in order to better reproduce the experimental population of discrete excited rotational bands feeding the SD yrast line, as shown in panel e). As already discussed in Sec. 4.1.1, this is consistent with the observation, in a number of SD nuclei (such as ${ }^{152} \mathrm{Dy}[152],{ }^{196} \mathrm{~Pb}[153]$ and $\left.{ }^{190,194} \mathrm{Hg}[154,17]\right)$, of strongly enhanced E1 transitions linking excited SD bands to the yrast band, with $\mathrm{B}(\mathrm{E} 1)$ values of the order of $10^{-4}$ W.u.. Such values are 1 to 2 orders of magnitude larger than expected from the tail of the GDR, used as standard parametrization of the E1 strength in the Monte Carlo simulation of the $\gamma$-decay flow.

Further insight on the $\gamma$-flow dynamics can be obtained from the analysis of the counts fluctuations in the $\gamma$-coincidence matrices (cfr. Sec. 2.3 [95]), allowing to estimate the number of discrete bands populating the ridges. In the case of ${ }^{151} \mathrm{~Tb}$, more than $30 \mathrm{SD}$ discrete bands, at least 2 steps long, are found to exist in the SD minimum, roughly half of which feed the SD yrast line, as shown on the right of Fig. 33, in agreement with the observed intensity of the ridge structures. The data can be compared with predictions by the simulation code MONTESTELLA: due to the use of microscopic levels, count fluctuations in simulated spectra not only reflect counting statistics but also the underlying nuclear structure and therefore, the extraction of the number of paths, in comparison with experimental data, represents a crucial test for various aspects of the model. The number of paths exploited by the $\gamma$-decay flux is, in fact, governed by the interplay between level density, band mixing, tunnelling through the 


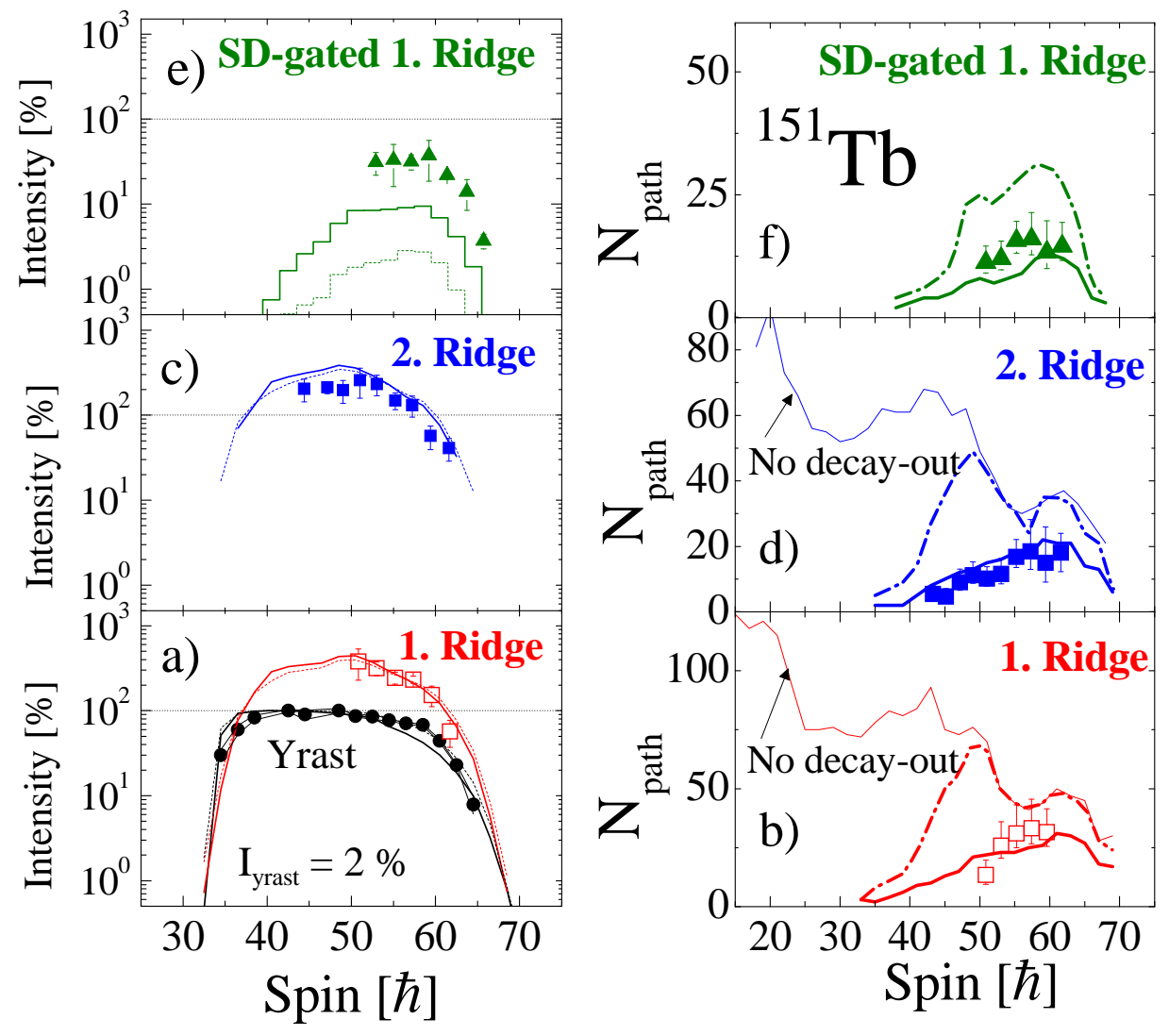

Figure 33: (Color online) Left column: Population of the excited rotational bands in the SD well of ${ }^{151} \mathrm{~Tb}$, relative to the corresponding SD yrast intensity (set to $100 \%$ ). Data (shown by symbols) give the intensities of the SD yrast band a), the 1st, the 2nd ridge and the SD-gated 1st ridge (a), c) and e), respectively). Predictions from simulations of the $\gamma$ decay assuming a standard (enhanced) E1 strength (cf. Fig. 27) are shown by dashed (solid) lines. Right column: Number of discrete excited SD bands obtained from the analysis of the count fluctuations of the ridge structures of ${ }^{151} \mathrm{~Tb}$. Symbols refer to data, thick solid lines to the Monte Carlo simulation. See the text for the thin and dashed lines (adapted from Ref. [75]).

barrier, etc. [73]. In the case of ${ }^{151} \mathrm{~Tb}$, the Monte Carlo calculations provide very good agreement with the data for the entire analysis of the ridge structures (thick solid lines in panels b), d) and f)). Moreover, the simulation allows for a further understanding of the flow-dynamics: the number of bands (paths) given by the simulation is much lower than the number of discrete bands (defined by the condition $n_{\mathrm{b}}<2$, cfr. Eq. 29), predicted by the cranked shell model, ignoring the tunnelling (thin solid lines in panels b) and d)). If the depopulation of the bands due to the tunnelling through the potential barrier between the ND and SD wells is considered, the number of bands decreases at low spins, where the decay out occurs, but still largely overestimates the data, as shown by dashed lines. A satisfactory agreement with the experimental values can only be obtained by properly taking into account the population of the excited bands, and this can only be done on basis of the Monte Carlo simulation. This is even more crucial than in the case of ND nuclei, since in the case of SD nuclei the work of Ref. [75, 93] shows that the population of discrete excited bands varies over more than 3 orders of magnitude, being very intense for the lowest lying bands, due to the rapid focusing of the $\gamma$-decay flow into the low excitation energy region above the SD yrast band. As a consequence, the corresponding number of bands detected by the fluctuation analysis (paths) is determined by the coldest bands which account for more than $90 \%$ of discrete bands population, at a given spin, while the numerous weak bands at higher excitation energies give almost no contribution to the final effective number of paths. 

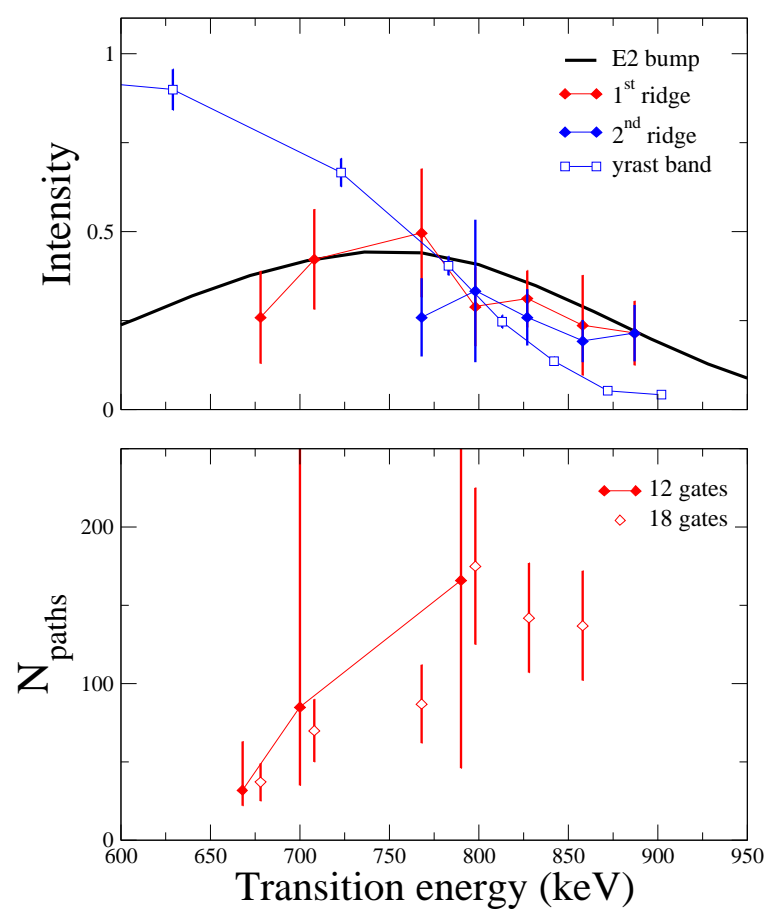

Figure 34: (Color online) Top panel: Intensity in units of multiplicity/30 keV present in the first and second ridges, the 1D quasicontinuum E2 distribution and the SD yrast transitions as a function of transition energy (where multiplicity is the number of transitions per cascade). Bottom panel: Number of 2-step decay paths contributing to the first ridge, obtained from different matrices requiring 12 or 18 gates on SD yrast transitions. The error bars from the SD-gated matrix with 12 gates are due to the large uncertainty in the determination of the second moment $\mu_{2}$ (adapted from Ref. [70])

The experimental results discussed above are quite similar to that observed in ${ }^{196} \mathrm{~Pb}$ [73] and in the partial analysis of ${ }^{134} \mathrm{Eu}[91,92],{ }^{132} \mathrm{Ce}[174]$ and ${ }^{152} \mathrm{Dy}$ [59]. This suggests that, in general, the population of the SD yrast band is due to both discrete and strongly mixed configuration, and that a significant fraction of SD discrete excited bands is not fully trapped in the SD minimum, but feeds directly the ND configurations, tunnelling through the potential barrier between the two wells.

A remarkable exception to the previous scenario is offered by ${ }^{194} \mathrm{Hg}$ [70], where exceptionally narrow $(\approx 10 \mathrm{keV})$ ridges were observed in coincidence with the SD yrast band, exhausting nearly all E2 decay strength and collecting the population of an extremely large number of discrete bands $(\geq 100)$, as shown in Fig. 34. This indicates that there is very little loss of rotational correlations in excited states in the SD well, although cranking calculations suggest that the wave function of these states is very complex, being made of a large number of components (with $n_{\text {mix }}$ up to 30, cfr. Fig. 26). Such a special situation has been interpreted as an example of ergodic nuclear system [96], namely a chaotic regime, where wave functions are complicated, representative of a chaotic system, and transition strengths exhibit PorterThomas fluctuations, yet collective rotation and flow are preserved. The occurrence of a similar scenario can not be fully excluded in the case of ${ }^{196} \mathrm{~Pb}$, since fission cuts off the high spin phase space much earlier than in ${ }^{194} \mathrm{Hg}$. This makes it impossible, in ${ }^{196} \mathrm{~Pb}$, to observe ridge structures beyond the crossing point between the ND and SD yrast lines (around $40 \hbar$ ), namely in the highest spin region where the effect was found in the case of ${ }^{194} \mathrm{Hg}$. Moreover, at low spins, where microscopic calculations predict large mixing numbers in ${ }^{196} \mathrm{~Pb}$, the extracted number of bands is influenced by the different population of excited bands, which bias the results towards lower values, as discussed above (cfr. Fig. 33) and in Ref. [75]. 
It is important to underline that the previous detailed analysis of SD ridge structures, in terms of intensities and count fluctuations, may provide a useful tool to investigate the properties of the potential barrier between the two wells (SD and ND), as a function of spin and excitation energy. Figure 35 illustrates, in the case of ${ }^{196} \mathrm{~Pb}$, the sensitivity to the barrier penetrability (depending on the $m_{0}^{(h o p)}$ mass parameter of Eq. 11, scaled by the $C_{m}$ constant in the model of Ref. [97]) for both the yrast and the excited SD bands. The dependence on spin can be tested by looking at the yrast pattern (panel a)), while the dependence on excitation energy can be investigated by looking at the intensity and number of paths populating the $1^{\text {st }}$ ridge. As shown in panel b), the intensity of the ridge structure is well reproduced by the MONTESTELLA calculations using the same value $C_{m}=2.5$ found for the SD yrast band (panel a)), therefore supporting a similar decay-out mechanism for the yrast band and the excited bands. The sensitivity to $C_{m}$ can be further tested by evaluating the number of excited bands: as shown in the top panel d) the number $N_{\text {band }}$ of excited bands, available to the $\gamma$ decay in the SD well, is strongly affected by the probability to decay out into the ND well, even after taking into account the bias given by the $\gamma$-decay flow (panel c)), which favours the population of the lowest excited bands, as shown by the MONTESTELLA simulation [75, 93]. This type of analysis, based on the investigation of detailed features of the SD ridge structure, provides a way to test basic elements of the tunnelling process, as a function of excitation energy. It is important to mention that various aspects of the decay-out process, such as the excitation energy of the SD yrast at which the decay out occurs or the line shape and intensity of the decay-out QC distribution from the yrast band, can also be successfully investigated by the KL_SD model of Lauritsen et al., which can perform a detailed analysis starting from 2-3 specific discrete states at the bottom of the yrast SD band, if requested. A notable example is the estimate of the excitation energy of the SD yrast band of ${ }^{192} \mathrm{Hg}$, reported in Ref. [141].

Complementary information on the nature of the QC components in the SD well can be obtained by other analysis methods. In first place, a lifetime analysis of the QC can be performed, to firmly establish the collectivity of rotational structures in the SD well. This type of study was performed in the case of ${ }^{134} \mathrm{Eu}$ [92]. As shown in Fig. 36, the fractional Doppler shift $\mathrm{F}(\tau)$ experimentally obtained for the $\mathrm{SD}$ yrast band (circles), the ridges (squares) and the E2 QC distribution (open diamonds) is consistent with a large quadrupole deformation, in agreement with the one of the SD yrast band, but very different from the triaxial case (triangles), the second largest known deformation in the ${ }^{134} \mathrm{Eu}$ nucleus, after the $\mathrm{SD}$ one. The analysis supports the SD nature of the excited rotational bands. This shows that the SD nucleus maintains its collectivity with increasing excitation energy, at least until rotational damping sets in, as predicted by the cranked shell model calculations of Yoshida et al. [92], and also observed in ND nuclei $[175,83]$. Second, angular distributions can be performed. This was done in the case of ${ }^{134} \mathrm{Eu}$ [163], ${ }^{132} \mathrm{Ce}$ [174], ${ }^{152} \mathrm{Dy}$ [59] and ${ }^{194} \mathrm{Hg}$ [15], confirming the E2 character of the rotational correlations in the SD well, up into the regime of the strongly interacting bands.

As a concluding remark on the issue of rotation at high temperature, it is worth mentioning that the investigation of QC spectra in one dimension, in particular as a function of angular momentum, is a powerful tool to enhance the sensitivity to properties of the warm rotational motion in deformed systems. In fact, in the case of rotors with stable deformation, the edge of the E2 distribution extends towards high $\gamma$-ray energy, with increasing angular momentum, a behaviour generally observed in ND rotational nuclei $[130,176,84]$.

In the case of highly deformed systems, such as ${ }^{134} \mathrm{Eu}$, this technique was used to prove the existence of a strong rotational continuum of SD nature, preferentially feeding the spherical configuration at low spins [163]. An additional case is given by the work of Ward et al. [177], where a transition towards a highly deformed Jacobi-like shape was inferred from the study of 1D QC distributions. As shown in Fig. 37, in systems like ${ }^{140} \mathrm{Nd},{ }^{108} \mathrm{Cd}$ and ${ }^{91} \mathrm{Nb}$ the nuclear moments of inertia (given by the slope of 


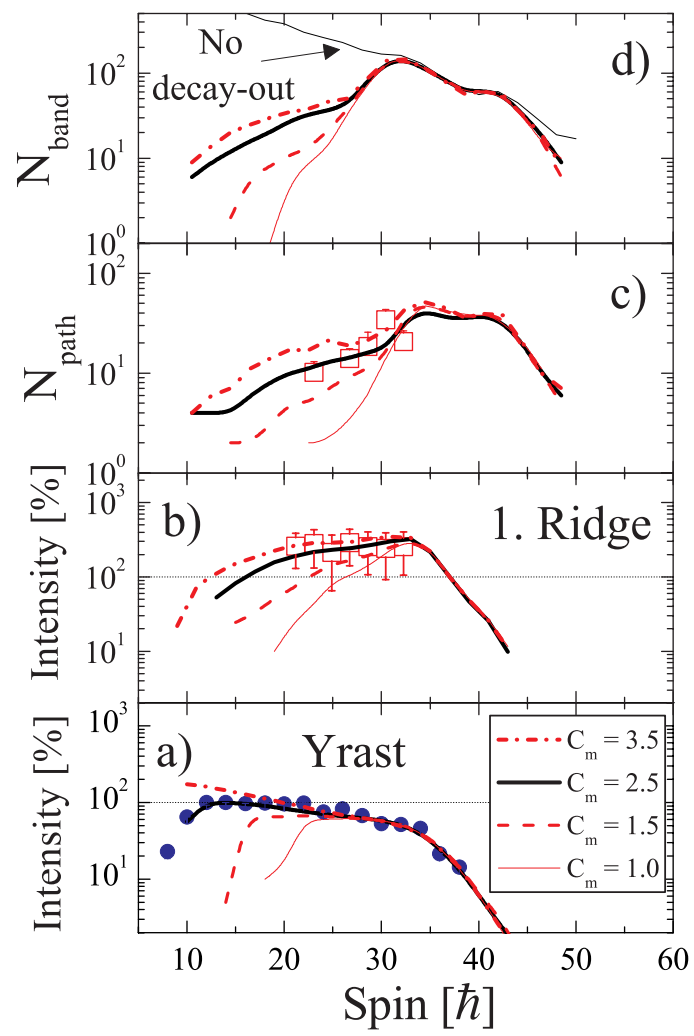

Figure 35: (Color online) Dependence of the results of the MONTESTELLA simulation of ${ }^{196} \mathrm{~Pb}$ on the scaling factor $C_{m}$ of the mass parameter (see Eq. 11), governing the tunnelling through the barrier. Panels a) and b) refer to the intensity of the SD yrast band and of the 1st ridge, panel c) to the number of paths populating the first ridge. The top panel d) gives, for the same mass parameters, the number of bands as obtained by the cranked shell model of Ref. [145] (thin solid line), taking also into account the probability to decay out into the ND well. Symbols refer to the corresponding experimental data $[75]$.

the spin versus frequency plot) tend to increase with increasing angular momentum up to the highest observed values. In addition, indication of a discontinuous change in the sign of the slope in the plot of frequency versus angular momentum is found at a critical angular momentum. This is where the triaxial Jacobi-like configurations is predicted to take over the McLaurin-like oblate shapes, as a consequence of the rapid increase in the fluid's elongation and moment of inertia, caused by the centrifugal force, once the Jacobi regime is entered. The phenomenon is often referred as "giant back-bending", since it appears, in the E2 QC 1D spectra, as a systematic decrease of $\mathrm{E}_{\gamma}=2 \hbar \omega$ with increasing spin. This observation can, therefore, be used as a signature of extreme deformation at high temperature.

As a final note, it is worth mentioning that information on the properties of the warm rotation in terms of rotational and compound damping widths (see Sec. 3.6) can be obtained by studying the spectral shape of rotational correlations in one and two dimensions. In particular, simulations are used to reproduce the experimental features and the most likely damping parameters are extracted. These techniques have not been used for SD nuclei, while they have been extensively applied to deformed rare earth nuclei $[137,138,178,139]$, providing typical values of the order of 20 and $200 \mathrm{keV}$ for the compound and rotational damping width, respectively, in the intermediate spin region of 30-40 $\hbar$. These values are in very good agreement with cranked shell model calculations which predict, for the SD nuclei ${ }^{151} \mathrm{~Tb}$ and ${ }^{196} \mathrm{~Pb}$, very similar compound widths, while rotational damping widths are generally expected 


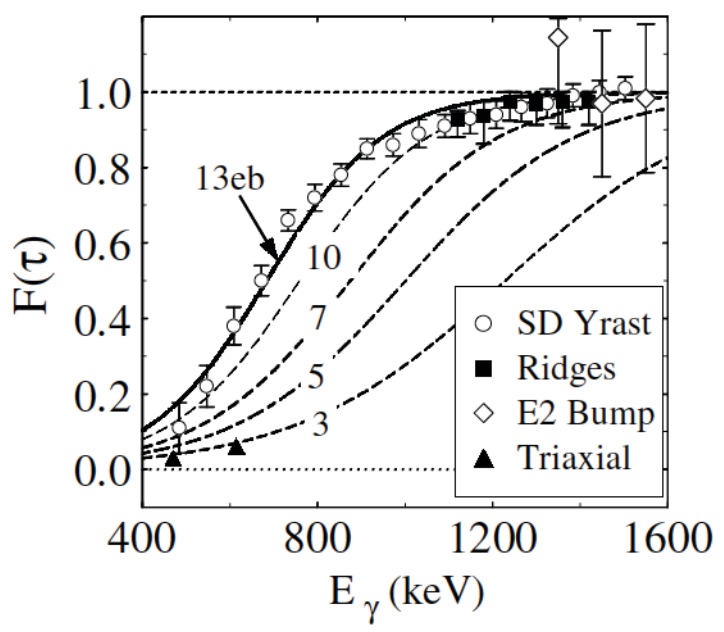

Figure 36: (The measured fractional Doppler shifts for the SD yrast band (circles), the SD ridges (squares), the E2 collective distribution (diamonds) and the triaxial transitions (triangles) experimentally observed in the nucleus ${ }^{134} \mathrm{Eu}$. The curves represent the expected theoretical values for the quadrupole moments $\mathrm{Q}_{t}=3,5,7,10$ and 13 eb (adapted from $[163,92]$ ).

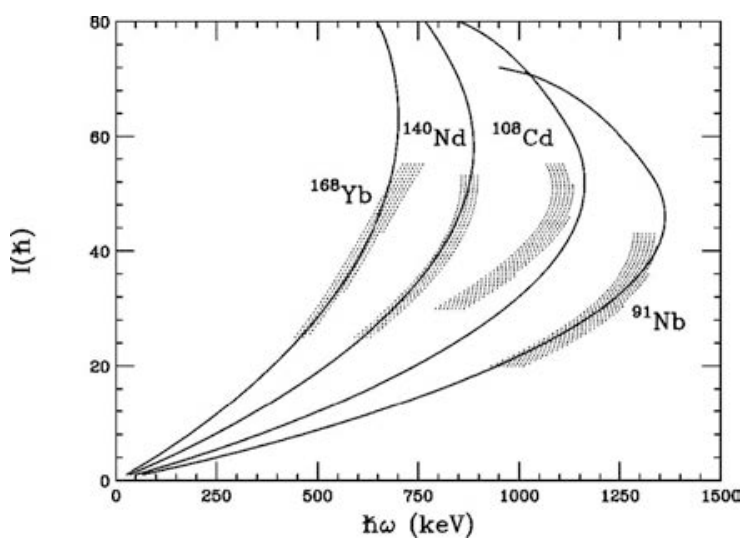

Figure 37: Plots of the experimentally derived spin versus rotational frequency (shaded areas). The dynamical moment of inertia $\Im^{(2)}$ is given by the slope $d I / d \omega$. Solid lines indicate results of ThomasFermi calculations which predict a Jacobi-like regime above a critical angular momentum value [177].

around $100 \mathrm{keV}$ at most. A notable exception is ${ }^{192} \mathrm{Hg}$, for which a significantly smaller value (of the order of $30 \mathrm{keV}$ ) is predicted for the rotational damping width, in agreement with the experimental observation of exceptionally narrow ridge structures in the neighbouring nucleus ${ }^{194} \mathrm{Hg}$, as discussed above [70].

\subsection{Decay from the superdeformed yrast line to the ground state}

SD states are found to decay mainly via $\gamma$ decay to states of smaller deformation with the notable exception of the actinide region, where SD states mainly decay by spontaneous fission [6]. In the late 1990's and early 2000's, it was discovered that SD states could also promptly decay by particle emission (proton or $\alpha$ ) $[27,28]$ into near spherical states of the daughter nucleus. This phenomenon has however, only been observed in proton-rich mass 60 nuclei.

As mentioned in the theoretical section of this review (Sec. 3), the necessary ingredient for there to be $\gamma$ decay out is a certain mixing with states at smaller deformations. It is the properties of these ND states, which govern the decay and in particular the shape of the decay spectrum. The 2 extreme cases 

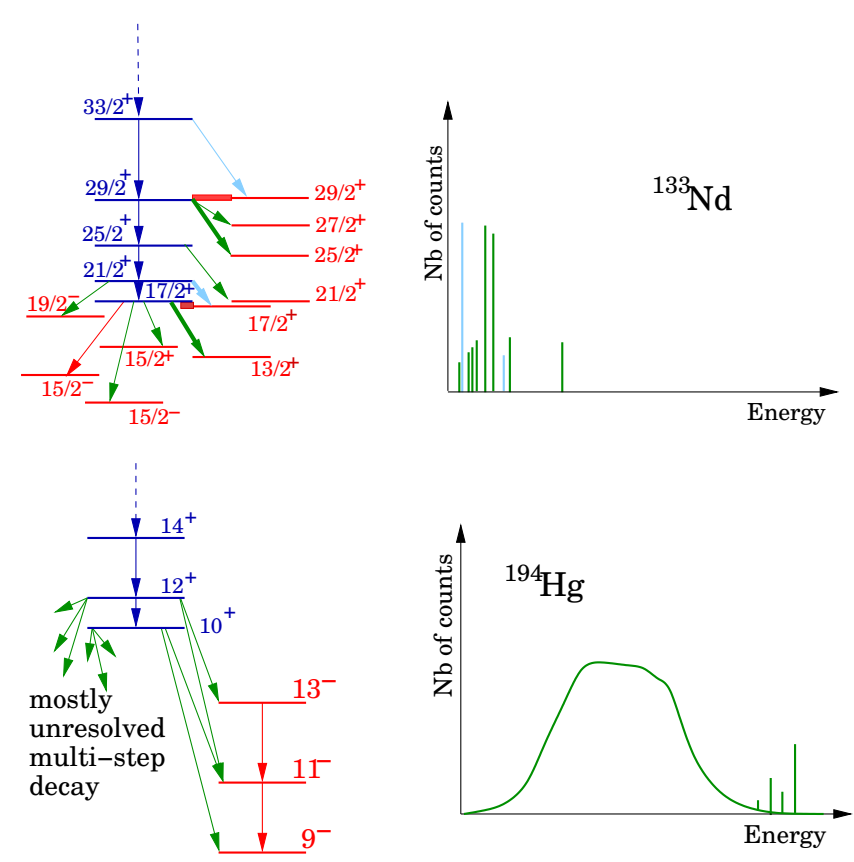

Figure 38: (Color online) Schematic illustration of the two extreme decay-out situations. Top) Decay scheme and corresponding decay-out spectrum of the yrast SD states of ${ }^{133} \mathrm{Nd}$. Bottom) Same as in the top for ${ }^{194} \mathrm{Hg}$. Blue transitions indicate SD transitions, while green transitions show the decay-out transitions. Blue(red) lines indicates states of predominant $\mathrm{SD}(\mathrm{ND})$ nature.

of decay-out situations are illustrated in the following and in Fig. 38. In the case of ${ }^{133} \mathrm{Nd}$ [179], where the difference in deformation between the ND states and the SD states is very small, the $\mathrm{I}=29 / 2$ and $17 / 2 \hbar \mathrm{SD}$ states are found to be nearly degenerate with states belonging to different ND bands. In fact, the SD and ND states interact strongly and repulse. This gives rise to three very strong transitions, which de-excite the SD band. In total, the decay spectrum is composed of 10 lines, which carry $100 \%$ of the SD band intensity. The yrast SD bands in ${ }^{132-137} \mathrm{Nd}$ have all been connected to the corresponding ND level scheme $[180,179,181,182,183,184]$. The value of the observed decay-out intensity is 100,50 and $20 \%$ for ${ }^{132} \mathrm{Nd},{ }^{134} \mathrm{Nd}$ and ${ }^{136} \mathrm{Nd}$ and 100,75 and $30 \%$ for ${ }^{133} \mathrm{Nd},{ }^{135} \mathrm{Nd}$ and ${ }^{137} \mathrm{Nd}$, respectively. This decrease is related to the increase in the excitation energy of the SD bands with neutron number: as $\mathrm{N}$ approaches 82, deformed structures are getting more costly in energy. Also, the absolute excitation energy is systematically higher in the even systems than in the odd ones. This is related to the nature of the SD configuration; two-quasiparticle in the even cases and one-quasi-particle in the odd cases. In the case of ${ }^{194} \mathrm{Hg}$ [185], the SD states mix with highly excited ND states. Unlike the case of ${ }^{133} \mathrm{Nd}$, one cannot resolve experimentally the states that mix at spins 12 and $10 \hbar$, where the decay out occurs, because they are only a few tens of eV apart (in $\mathrm{Nd}$, the states, which mix are 32 and $46 \mathrm{keV}$ apart before interaction). Since the excitation energy is very large, the phase space available for the decay is huge. The corresponding spectrum, very different to the one in ${ }^{133} \mathrm{Nd}$, is a QC spectrum, on which are superimposed some resolvable lines, in particular, the ones that connect the SD states to the known ND yrast line. These single-step links account for just over 3\% of the SD band intensity. In total, less than $15 \%$ of the decay-out flux is resolved.

\subsubsection{Single-step links}

One can unambiguously determine the absolute energy, spin and parity of SD states by identifying directly connecting pathways from the SD state to known ND states. However, despite enormous experimental efforts, only a handful of SD bands have been connected to the low-lying ND states. This 

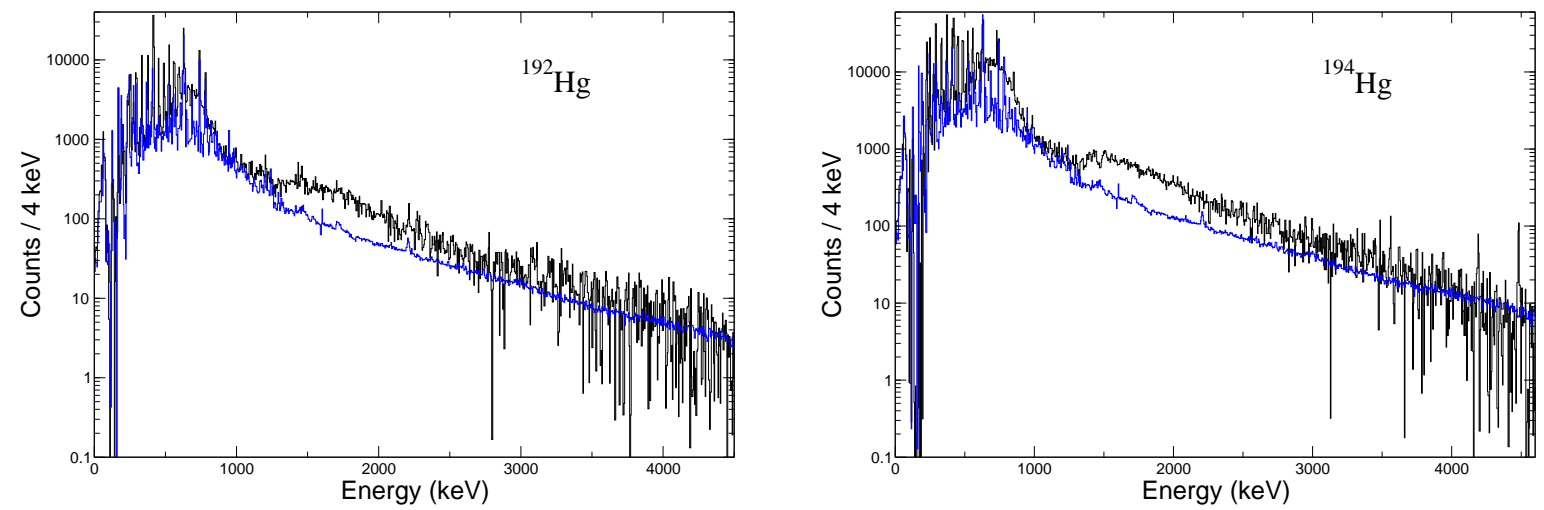

Figure 39: (Color online) Left (Right): Black) spectrum of transition in coincidence with at least 2 SD transitions of ${ }^{192} \mathrm{Hg}\left({ }^{194} \mathrm{Hg}\right)$. Blue) spectrum of transition in coincidence with low-lying ND transitions of ${ }^{192} \mathrm{Hg}\left({ }^{194} \mathrm{Hg}\right)$. The spectra are normalised to the same number of counts above $4 \mathrm{MeV}$.

is because in most of the cases, the density of final states for the decay is very large. The decay is consequently highly fragmented. Moreover, the transitions which directly connect SD states to lowlying ND states are high-energy $\gamma$-rays, with a reduced efficiency for detection and they also suffer from Porter-Thomas strength fluctuations (see Sec. 4.2.3). It is therefore a difficult experimental task to observe these single-step links since their signal corresponds to $\sim 10^{-4}$ of the reaction channel. The spectrum of Fig. 39 shows the unfortunate example of ${ }^{192} \mathrm{Hg}$, for which no single-step links have been found. Indeed, it resembles in every way the equivalent spectrum of ${ }^{194} \mathrm{Hg}$ shown on the right of Fig. 39, with the notable exception of the sharp lines at the very top of the spectrum (the single-step links).

In some cases, nevertheless, nature is kind and single-step links can be seen at the very top of the decay spectrum. Only for a handful of nuclei, however, has this been possible and Tab. 1 lists the isotopes for which absolute excitation energies have been obtained for SD states thanks to the observation of single-and/or double-step links. In this last case, some results should be taken with caution. Indeed, when the excitation energy of the decaying SD state is large, the large level density of intermediate states makes it highly unlikely that a particular 2-step cascade be more likely than any other.

Experimental techniques, such as isomer tagging are very useful in reducing the amount of contamination from other reaction channels and therefore in identifying single-step links and determining their coincidence relationships with ND transitions. This method has been used both in the mass 150 and 190 regions $[152,186,187,188]$. Searching for single-step links is a difficult task and it may happen that some lines, which appear to be real in a one experiment are no longer visible in another (see eg. the case of ${ }^{193} \mathrm{Tl}$ decay out lines $\left.[189,190]\right)$. This tells us that caution is needed when treating peaks at the very limit of the resolving power of multidetector arrays and that setting a $3 \sigma$ limit on the intensity of lines may not be, in some cases, a sufficient criterium.

In the cases where no obvious single-step links could be identified, 2-step transitions were searched by using the summing technique $[191,192]$. The idea behind this approach is that even though the density of intermediate levels is high, the sum of the energies involved in 2-step cascades should be sharply defined since they reflect the difference in energy between the initial SD and final ND states. No conclusive results, however, could be obtained.

As mentioned previously, the decay from SD states occurs because of mixing with ND states through a potential barrier: the SD wavefunction acquires an amplitude $\alpha^{2}$ at normal deformation. The SD state has a partial width $\left(1-\alpha^{2}\right) \Gamma_{\gamma, S D}$ to decay to the next SD state and a partial width $\alpha^{2} \Gamma_{\gamma, N D}$ to decay to lower energy ND states. If the coupling between ND and SD states is weak, the mixing will 


\begin{tabular}{|c|c|c||c|c|c|}
\hline Nucleus & $(U$ in MeV,I, $\pi)$ & Ref. & Nucleus & $(U$ in MeV,I, $)$ & Ref. \\
& & & & & \\
\hline${ }^{238} \mathrm{U}$ & $(2.56,0,+)$ & {$[193]$} & ${ }^{133} \mathrm{Nd}$ & $(0.53,17 / 2,+)$ & {$[179]$} \\
\hline${ }^{236} \mathrm{U}$ & $(2.75,0,+)$ & {$[194]$} & ${ }^{134} \mathrm{Nd}$ & $\begin{array}{c}(0.59,17,-) \\
(1.2,18,+)\end{array}$ & {$[181]$} \\
& & & & $(181]$ \\
\hline${ }^{192} \mathrm{~Pb}$ & $(2.12,8,+)$ & {$[186]$} & ${ }^{135} \mathrm{Nd}$ & $(0.52,25 / 2,+)$ & {$[182]$} \\
\hline${ }^{194} \mathrm{~Pb}$ & $(2.74,6,+)$ & {$[94,195]$} & ${ }^{136} \mathrm{Nd}$ & $(0.99,17,-)$ & {$[183]$} \\
\hline${ }^{196} \mathrm{~Pb}$ & $(3.43,6,+)$ & {$[187]$} & ${ }^{137} \mathrm{Nd}$ & $(0.77,29 / 2,+)$ & {$[184]$} \\
\hline${ }^{190} \mathrm{Hg}$ & $(2.82,12,+)$ & {$[188]$} & ${ }^{84} \mathrm{Zr}$ & $(2.78,25,-)$ & {$[196]$} \\
\hline${ }^{191} \mathrm{Hg}$ & $(3.14,31 / 2,(-))$ & {$[156]$} & ${ }^{60} \mathrm{Zn}$ & $(4.33,8,+)$ & {$[197]$} \\
\hline${ }^{194} \mathrm{Hg}$ & $(4.05,8,+)$ & {$[185]$} & ${ }^{58} \mathrm{Cu}$ & $(3.57,9,+)$ & {$[198]$} \\
& $(5.09,9,-)$ & {$[17]$} & & & {$[129]$} \\
\hline${ }^{163} \mathrm{Lu}$ & $(0.99,13 / 2,+)$ & {$[199]$} & ${ }^{59} \mathrm{Cu}$ & $(2.24,25 / 2,+)$ & {$[201]$} \\
\hline${ }^{164} \mathrm{Lu}$ & $(1.13+\mathrm{x}, 13,+)$ & {$[200]$} & ${ }^{56} \mathrm{Ni}$ & $(2.13,7, ?)$ & \\
\hline${ }^{152} \mathrm{Dy}$ & $(1.28+\mathrm{x}, 14,-)$ & {$[200]$} & & & {$[28]$} \\
& $(3.41,24,+)$ & {$[152]$} & ${ }^{58} \mathrm{Ni}$ & $(1.94,15,-)$ & \\
\hline${ }^{149} \mathrm{Gd}$ & $(4.12,47 / 2,-)$ & {$[203]$} & ${ }^{40} \mathrm{Ca}$ & $(5.21,0,+)$ & {$[204]$} \\
\hline${ }^{151} \mathrm{~Tb}$ & $(3.58,65 / 2,+)$ & {$[205]$} & ${ }^{36} \mathrm{Ar}$ & $(4.33,0,+)$ & {$[206]$} \\
\hline${ }^{132} \mathrm{Nd}$ & $(0.85,9,-)$ & {$[180]$} & & & \\
\hline
\end{tabular}

Table 1: Table showing the nuclei for which the absolute excitation energy $U$ above the ND yrast line, spin $I$ (and parity $\pi$ ) of the last observed SD states are known. The values have been taken from [207] and references shown in the table.

result in two states which are populated from the previous SD state proportionaly to their SD amplitude squared: $\left(1-\alpha^{2}\right)$ for the predominently SD state and $\alpha^{2}$ for the predominantly ND state. SD transitions populating SD decaying states should then effectively be doublets. This is clearly observed in the mass 130 [179] and mass 160 [199] regions. The yrast SD states in ${ }^{163} \mathrm{Lu}$ (band TSD1) to which the excited, so-called "wobbling" band [23] decays, have been connected to the lower lying states [199]. In fact, there is a very strong interaction between the SD state and a neighbouring ND state at spin $21 / 2 \hbar$. This leads to irregularities in the behaviour of the moment of inertia $\Im^{(2)}(\omega)$. The SD $21 / 2^{+}$state acquires a small admixture of the ND $21 / 2^{+}$state and vice versa. The SD transition $25 / 2^{+} \rightarrow 21 / 2^{+}$is actually split into two transitions of 315 and $427 \mathrm{keV}$, respectively. The ND and SD $21 / 2^{+}$states will be fed from the $25 / 2^{+}$SD state proportionally to their squared SD amplitudes, $\alpha^{2}$ and $\left(1-\alpha^{2}\right)$ respectively. From the experimental intensity ratios, a value of $\sim 4 \%$ was extracted for $\alpha^{2}$. This is of the order of what one obtains in the mass 190 and 150 regions assuming a statistical decay. The unperturbed energies of band TSD1 can then be derived and the dynamical moment of inertia recalculated. As in the case of ${ }^{133} \mathrm{Nd}$, the irregularities of the moment of inertia disappear. The interaction strength between the SD and ND states is large $(22 \mathrm{keV})$. Similar values are found in the Nd isotopes.

In the mass 190 region, the splitting of a SD transition has been observed in ${ }^{194} \mathrm{~Pb}$ by using Low Energy Photon Spectrometers in conjunction with large volume HPGe detectors [208]. A detailed analysis of the line shape of the last SD transition at $169 \mathrm{keV}$, has revealed the presence of a low-energy shoulder. The ratio of the intensities of the main SD $8^{+} \rightarrow 6^{+}$peak and of its satellite yields a $15(5) \%$ ND admixture into the $6^{+}$SD state. This fits well with what can be estimated from the ratio of the inand out-of-band decay intensities at the $6^{+}$state and assuming a statistical decay out of the band.

The energy separation between the two peaks is $1.3(2) \mathrm{keV}$ after interaction and $0.9(2)$ before 
interaction. This is in line with the average level spacing between ND $6^{+}$states at $2.74 \mathrm{MeV}$ above the yrast line. The interaction strength is extracted to be $0.6(2) \mathrm{keV}$. The interaction is smaller than in the mass 130 and 160 region. This is because the difference between ND and SD configurations is much larger and many more particles have to be rearranged to go from one configuration to the other.

This rearrangement of particles involved in the decay-out process is also nicely supported by the observations of scaled decay-out strengths in ${ }^{58} \mathrm{Cu}$ [198]. Indeed, the E2 strengths of the 830, 1519 and $4171 \mathrm{keV}$ transitions depopulating the $\left(11^{+}\right)$second minimum state in ${ }^{58} \mathrm{Cu}$ scale as $0.1^{N} ; \mathrm{N}=0,2,4$ being the number of particle-hole excitations which are to be reconfigured. This is also what can be inferred from the observed correlation between the difference in quadruple deformation among SD and ND states and the rotational frequency at which decay out occurs [209].

In ${ }^{59} \mathrm{Cu}$, a large number of linking transitions have been observed to connect SD states to ND states [210]. These account for $\sim 90 \%$ of the total decay out intensity. With the help of a theoretical model to compute the interaction between the SD states and bands calculated by the configuration-dependent cranked Nilsson-Strutinsky (CNS) approach [211], the decay-out intensity pattern and spectrum could satisfactorily explained in terms of the coupling of the SD state with a small number of doorway states [129]. In the mass 150 and 190 region, these doorway states are mixed into the dense background of ND states. The question that can then be addressed is whether the decay has reached the statistical limit.

\subsubsection{Primary transition strengths}

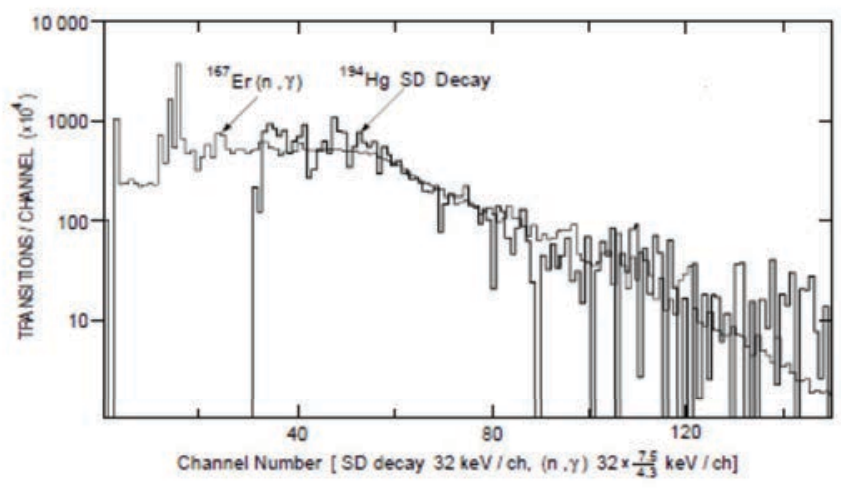

Figure 40: Overlay of spectra from the decay out of ${ }^{194} \mathrm{Hg}$ and from neutron capture on ${ }^{167} \mathrm{Er}$. The thermal-neutron capture in ${ }^{167} \mathrm{Er}$ is from a Argonne-Brookhaven-Manchester collaboration [33, 212]. The spectra have been adjusted so that their endpoints coincide.

Since it is the ND component of the SD state wavefunction which governs the decay out, asking whether the decay has reached the statistical limit is equivalent to asking whether the ND states to which the SD states couple through the potential barrier are compound states or not. There are many experimental observations which support this (the large excitation energy of SD states at the point of decay (see examples in Tab. 1), the QC nature of the decay spectrum $[122,60,59,156,213,78,91]$, the large fragmentation of the decay cascades [214], the fact that statistical decay calculations reproduce the general shape of the decay spectrum [95] and the similarity between the decay spectrum and the spectrum of $\gamma$-rays following resonant neutron capture at 7-8 MeV above yrast [215] (see Fig. 40). In the neutron resonance case, the statistical nature of the decay is well established [216]: the strengths of the primary transitions follow a Porter-Thomas distribution [217]. The particularity of the PorterThomas distribution is that unlike other $\chi^{2}$ distributions with higher degrees of freedom, it diverges towards low strengths, yet it also extends to very large strengths. This property gives rise to very 
strong intensity fluctuations which are clearly visible in Fig. 41. The figure shows the decay spectrum of $1^{-}$resonance states in ${ }^{196} \mathrm{Pt}$ [216] and of particular interest is the hugely varying intensity, from one resonance to the next, of the primary lines connecting the resonance state to the ground state and to the first 2 excited states of ${ }^{196} \mathrm{Pt}$. This is the direct consequence of Porter-Thomas fluctuations. These stochastic fluctuations could also be the reason why single-step decay transitions are sometimes enhanced and can be observed experimentally. This is the case in ${ }^{194} \mathrm{Hg}$, for which strong high-energy links have been identified while in the neighbouring ${ }^{192} \mathrm{Hg}$, studied with similar statistics, no such lines could be observed.

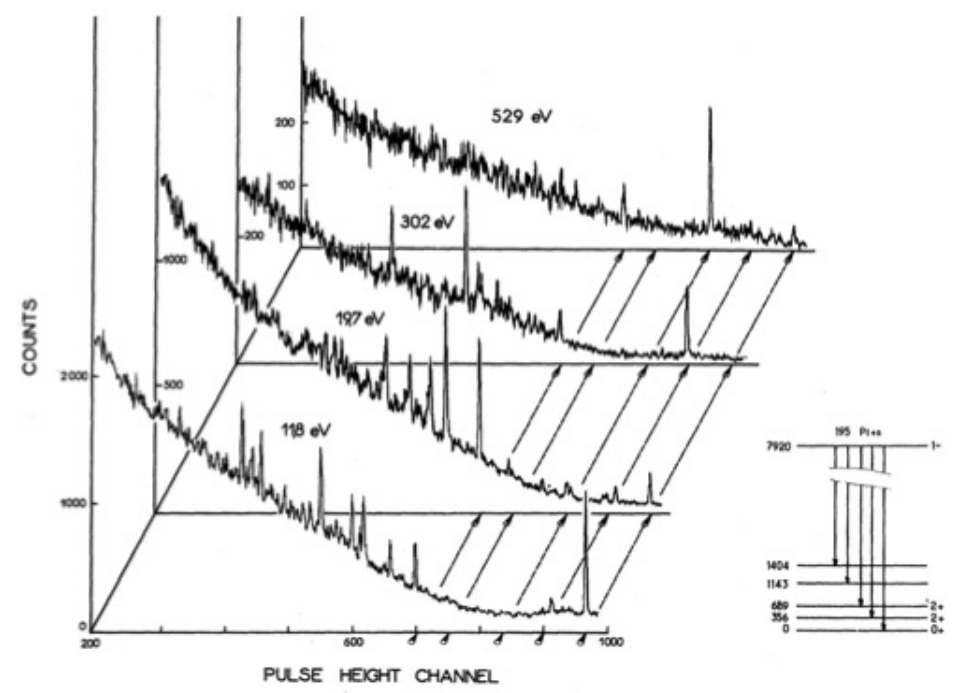

Figure 41: $\gamma$-decay spectra following resonant neutron capture on ${ }^{195} \mathrm{Pt}$ for different incident neutron energies [216]. The arrows show the position of the primary transitions connecting the $1^{-}$resonance states to the ground state and low-lying excited states in ${ }^{196} \mathrm{Pt}$.

In order to show that the enhancement of the strengths in ${ }^{194} \mathrm{Hg}$ may be due to Porter-Thomas fluctuations, a study of the primary strength distribution was performed. The aim was to determine which $\chi^{2}$ distribution $f_{\theta, \nu, \omega_{l o w}}(\omega)$ of $\nu$ degrees of freedom and average strength $\theta$ could best fit the experimental strengths $\omega_{i}$ extracted above the experimental strength threshold $\omega_{\text {low }}$ :

$$
f_{\theta, \nu, \omega_{l o w}}(\omega)=a\left(\theta, \nu, \omega_{\text {low }}\right) \omega^{\nu / 2-1} \exp \left(\frac{-\nu \omega}{2 \theta}\right), \omega_{\text {low }}<\omega<\infty
$$

where $a\left(\theta, \nu, \omega_{\text {low }}\right)$ ensures the proper normalisation. This was investigated using the Maximum Likelihood Method, i.e the most likely parameters are the ones which maximise the joint probability $L$ :

$$
L\left(\theta, \nu, \omega_{\text {low }}\right)=\prod_{i} f_{\theta, \nu, \omega_{\text {low }}}\left(\omega_{i}\right)
$$

of observing the $\mathrm{n}$ experimental strengths $\omega_{i}$. The result for the most likely $\chi^{2}$ distribution is the following: $\nu=1_{-1}^{+20}$ and the average strength $\theta$ is found to be nearly four times smaller than the experimental strength threshold [140]. The uncertainty on the number of degrees of freedom $\nu$ is very large because only the high-strength tail of the distribution is accessible experimentally and this is a strength domain for which there is not a pronounced difference between $\chi^{2}$ distributions. In other words, only the strongest 19 strengths are observed, whereas a fluctuation analysis in the same transition energy interval $\left(\mathrm{E}_{\gamma}>2.6 \mathrm{MeV}\right)$ tells us there should be $\sim 600$ [214]. For ${ }^{194} \mathrm{Hg}$, it was concluded that observed primary transitions might consist of the strongest transitions selected from a Porter-Thomas distribution. It should be pointed out, however, that the observation of 2 single-step linking transitions 
carrying more than 8 times the average strength $\theta$ is highly unlikely and this could imply that the decay in ${ }^{194} \mathrm{Hg}$ has not reached the chaotic limit.

The problem when dealing with an analysis in terms of $\chi^{2}$ distributions is the fact that one doesn't know what $\nu \neq 1$ means. To resolve this issue, a new method was developed to study the strength distribution of primary $\gamma$ rays in any type of regime, from ordered to fully chaotic, by introducing a chaoticity parameter [38]. This is no longer analytical and requires simulations. In the simulations, the SD state couples weakly only to a specific ND state, which acts as a doorway state. The spectrum of ND states is described by a large GOE matrix of size $N \times N$, the off-diagonal elements being multiplied by a chaoticity parameter $\Delta$. In this way, the structure of the ND states can be smoothly changed from regular, with $\Delta=0$, to chaotic, with $\Delta=1$. Via the interaction with the doorway state, the SD state acquires a ND admixture. When $\Delta=0$, only the doorway contributes to this admixture, while when $\Delta=1$, all the $\mathrm{N}$ basis states contribute with an admixture of the same order. This is what is called "chaos-assisted" tunnelling. To analyse the strength distribution in terms of $\Delta$, the conjecture is that admixtures can be viewed as strengths. For a given set of $N_{0}$ experimental strengths, the aim is to find the values of the parameters $\Delta$ and total number of sampled strengths $N_{t}$, of which only the $N_{0}$ strongest are visible (this sets the equivalent of an experimental strength threshold), which maximize the likelihood function. For ${ }^{194} \mathrm{Hg}$, the properties of the likelihood function tells us that $\Delta$ is probably larger than 0.1 and the 19 visible transitions are the strongest out of more than 200, the majority of them being hidden in the noise of the spectrum [208]. These limits are in agreement with the measurement of $\sim 600$ primary lines above $2.6 \mathrm{MeV}$ and with the result of the most likely $\chi^{2}$ distribution and provides strong evidence that the decay out of SD states in ${ }^{194} \mathrm{Hg}$ behaves very much like the decay of a compound state.

\subsubsection{Decay-out quasicontinuum}

The mass 190 region is so far the only mass region where the QC decay-out spectrum can be extracted experimentally by disentangling it from the feeding components (cf Sec. 2). In this subsection we will

summarise what has been learnt from studying the (i) fluctuations and (ii) shape and intensity of this QC decay-out spectrum.

\section{(i) Fluctuations of the decay-out spectrum}

The fluctuations of the decay-out spectrum were studied in the case of ${ }^{192} \mathrm{Hg}$ [81] according to the prescriptions described in section 2.3. Since the spectrum in coincidence with SD yrast lines in ${ }^{192} \mathrm{Hg}$ above $1 \mathrm{MeV}$ is composed of the SD decay-out spectrum as well as the underlying continuous statistical feeding spectrum, one needs to determine the fraction $f$ of events belonging to the SD band decay. The best experimental approximation of the spectrum of statistical $\gamma$-rays feeding the SD band above 1 $\mathrm{MeV}$ is taken to be the spectrum of events in coincidence with low-lying ND lines (see the blue spectrum of Fig. 39). $f$ can then be computed as a function of $\gamma$-ray energy. Applying Eq. 6, one then finds that $\sim 10000$ transitions are sampled in the decay out of the SD band in ${ }^{192} \mathrm{Hg}$ (see Fig. 42). Above $2 \mathrm{MeV}$, fewer transitions are sampled by the nucleus. This can be explained by the fact that these transitions are primary $\gamma$-rays, starting off from a few initial states and taking the nucleus down to regions of the (I, E) plane of low level density. Thus, for the decay cascade, the number of transitions is expected to decrease at high transition energy in proportion to the final state level density $\rho\left(U_{\text {final }}\right)$. On the other hand, the fluctuation analysis carried out on the experimental approximation of the feeding spectrum gives a different behaviour at high energy: In this case, the number of transitions reflects the product of the final and initial state level densities $\rho\left(U_{\text {initial }}\right) \rho\left(U_{\text {final }}\right)$.

In panel b) of Fig. 42, the experimental effective number of transitions is compared to theoretical statistical calculations based on schematic equidistant levels with pairing included [95]. In this schematic 


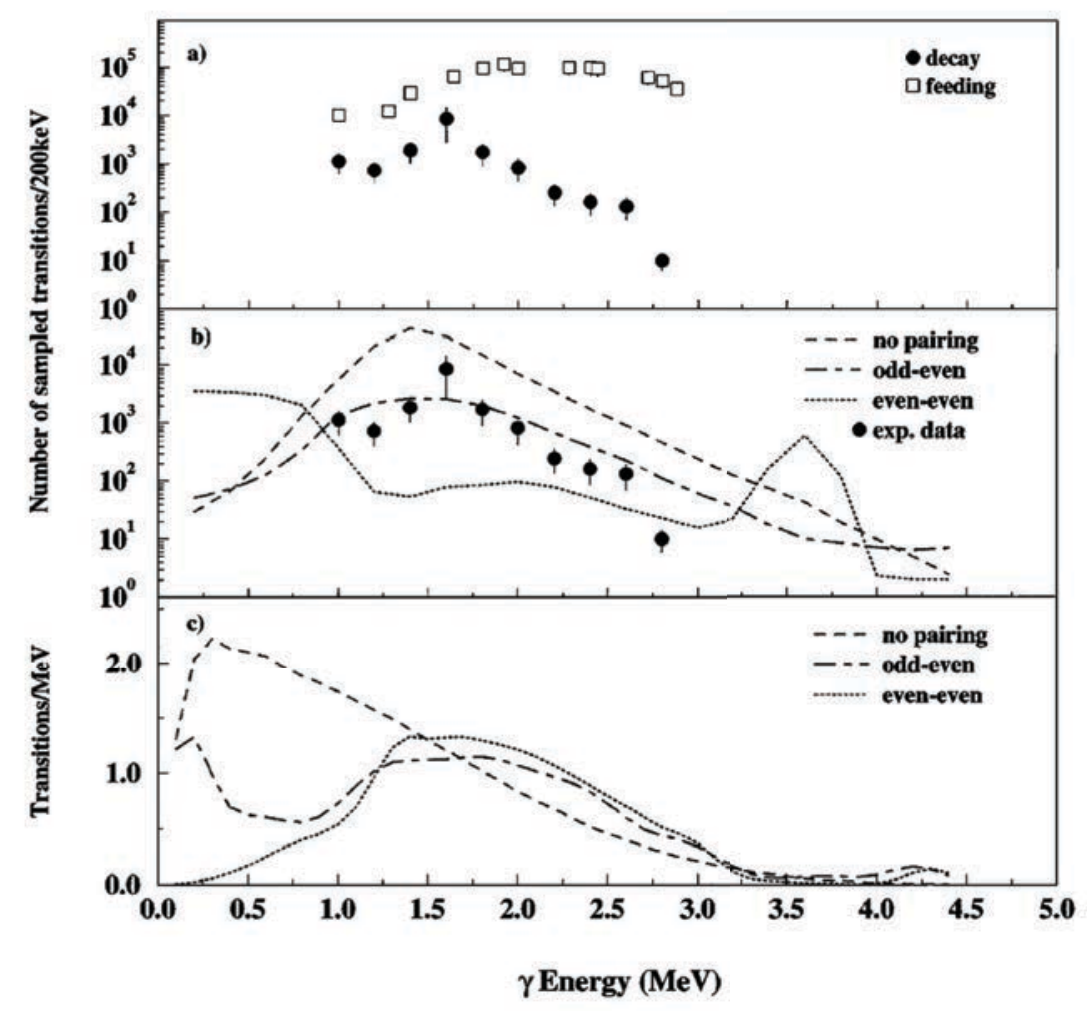

Figure 42: Upper panel (a) Experimental number of paths sampled by the nucleus in the feeding (open circles) and in the decay-out cascades of SD states in ${ }^{192} \mathrm{Hg}$. Middle panel b) Comparison between the experimental number of paths sampled in the decay-out cascade and calculations assuming a statistical decay from a $4.3 \mathrm{MeV}$ state and no pairing or pairing appropriate for an even-even and odd-even nuclei. Lower panel c) Calculated statistical spectra for the cases described in b) Ref. [81].

model, quasiparticle energies are calculated, starting from equidistant single-particle levels, with pairing treated using the BCS method, followed by particle-number projection and diagonalization.

This is done in the case of an unpaired even-even nucleus and paired odd-even and even-even nuclei. The comparison between the experimental data and the calculations is satisfactory and is a stringent test for calculations of the decay and of the density of ND states $\rho(U)$. The fact that the experimental values are in better agreement with the odd-even calculated values may be due to factors that have not been included in the model: first of all, angular momentum which causes the pairing correlations in the even-even nucleus to weaken and hence lowers the ground state pairing gap. Secondly, shell effects may slightly displace both calculated curves. Thirdly, the use of equidistant levels is an approximation to the true level distribution. There is a need for a proper comparison of theory and experimental data, especially for an odd case.

\section{(ii) Shape of the decay-out spectrum}

The shape of the decay-out QC spectrum is consistent with the statistical decay of a compound nucleus. In Fig. 40 the spectrum from the decay out of ${ }^{194} \mathrm{Hg}$ is overlaid with the spectrum from neutron capture on ${ }^{167} \mathrm{Er}$. In both cases, the decay originates from a discrete point in excitation energy and spin and proceeds to a multitude of final states. The neutron capture spectra is known to be statistical. The shapes and multiplicities of the spectra are strikingly similar, so in combination with other arguments given earlier in this section, this corroborates that we are seeing statistical or compound 


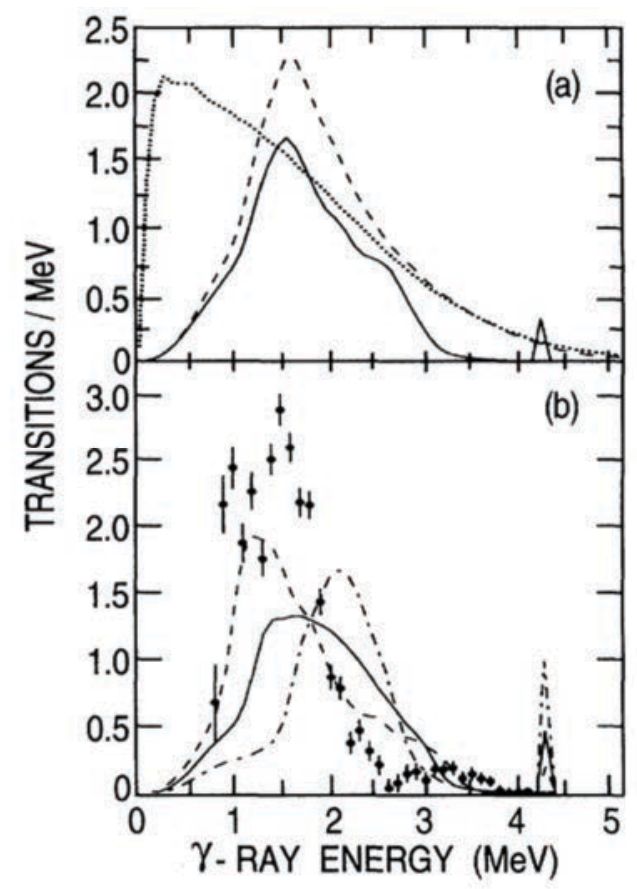

Figure 43: (a) Calculated statistical decay spectrum from an initial state in ${ }^{192} \mathrm{Hg}$ at $\mathrm{U}=4.3 \mathrm{MeV}$ (solid line) and $U=7.5$ (dashed line) excitation energy above the yrast line, using level densities obtained from BCS quasiparticle states. The dotted curve shows the spectrum with no pairing for U=7.5 MeV. The excitation energy of $4.3 \mathrm{MeV}$ in ${ }^{192} \mathrm{Hg}$ is taken from the QC analysis of Henry et al. [122]. (b) Same as (a), but for level densities from diagonalised states for $\mathrm{U}=4.3 \mathrm{MeV}$, with three different strengths of the pairing interaction, parametrised by the ground state pairing gaps $\Delta_{0}=0.7,0.9$, and $1.1 \mathrm{MeV}$ (dashed, solid, and dot-dashed lines, respectively) [95]. For comparison, the dots with error bars show the experimental decay-out spectrum for ${ }^{192} \mathrm{Hg}$ [122].

nucleus decay. The spectrum has a bell shaped form characteristic of statistical decay, reflecting the two factors that influence the decay, namely the level density and the $\gamma$-strength function. The level density increases exponentially and would therefore increasingly favour low-energy transitions. The $\gamma$-strength function is dominated by the tail of the GDR, and falls off exponentially as the $\gamma$ energy decreases, favouring high-energy $\gamma$ rays. So, folding the two competing effects together results in a spectrum with a bell shaped form.

The shape of the decay-out spectrum has been calculated theoretically in Ref. [95] and the effect of including pairing (or not) in the ND stated has been investigated. The bottom panel of Fig. 42 shows that the shape of the calculated decay-out spectrum, from $\mathrm{U}=4.3 \mathrm{MeV}$ for ${ }^{192} \mathrm{Hg}$, changes drastically when including pairing compared to not, while the difference between using pairing appropriate for even-even and odd-even nuclei is biggest below $\gamma$-ray energy $E_{\gamma}=1 \mathrm{MeV}$. The results, shown in Fig. 43 panel (a), is that the shape of the calculated decay-out spectrum, from $\mathrm{U}=7.5 \mathrm{MeV}$ for ${ }^{192} \mathrm{Hg}$, also changes dramatically if ones includes pairing compared to not, corresponding to the dashed and dotted curves, respectively. For the solid line in panel (a), also calculated with pairing, but now $\mathrm{U}=4.3 \mathrm{MeV}$, the endpoint of the spectrum is seen with a peak corresponding to direct decay to the ND yrast state and then a gap in the spectrum with no counts, reflecting a region with no levels to decay to and whose extension depends on the size of the pairing gap.

In Fig. 43 panel (b) the experimental decay-out spectrum for ${ }^{192} \mathrm{Hg}$ [122] is compared to theoretical calculation [95] using different ground state pairing gaps $\Delta_{0}$. The best agreement is obtained with a ground state pair gap $\Delta_{0}=0.7 \mathrm{MeV}$, which is below the expected value of $0.9 \mathrm{MeV}$. This is probably 
due to the quenching of the pair correlations by angular momentum, which is around $8 \hbar$.

Experimentally, information on the pair gap in ND states can be extracted from the above mentioned gap in the decay-out spectrum below the endpoint. This was done in Ref. [213] for ${ }^{194} \mathrm{~Pb}$ and ${ }^{192} \mathrm{~Pb}$ for which SD excitation energies and spins are known [186, 94, 195]. The different unresolved feeding and decay-out components were found using the method outlined in Sec. 2 and are shown in panel (a) of Fig. 44 [213]. In Fig. 44 are also shown the measured decay-out spectrum for the yrast SD band

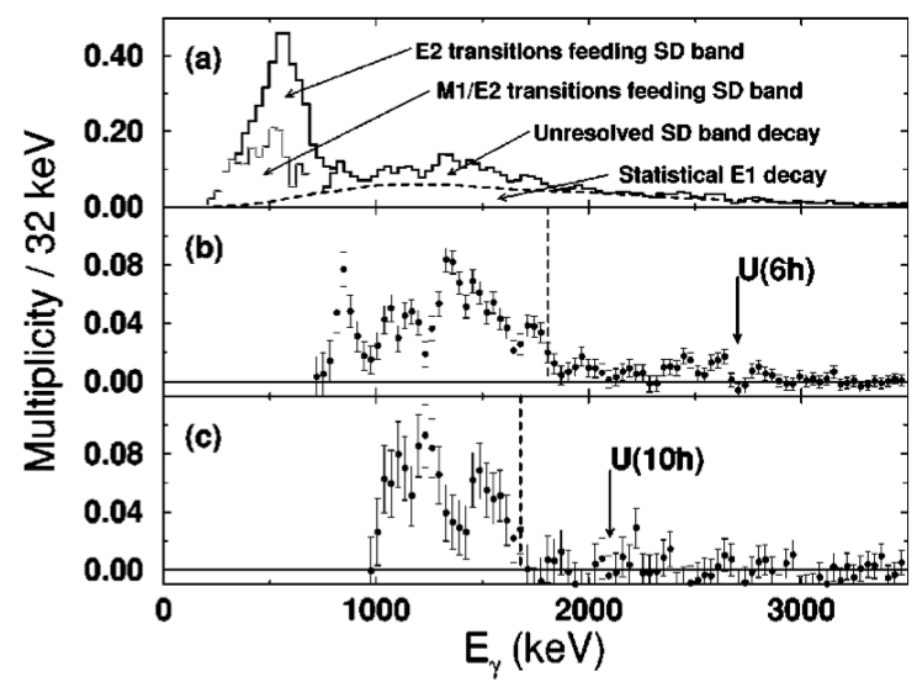

Figure 44: Panel a) Decomposition of the SD-gated spectrum in ${ }^{194} \mathrm{~Pb}$. Panel b) Extracted Decay-out spectrum of SD states in ${ }^{194} \mathrm{~Pb}$. Panel c) Same as b) for ${ }^{192} \mathrm{~Pb}$. The vertical dotted lines indicate where the decay-out QC spectrum vanishes. The energy interval between this point and the end-point of the decay-out spectrum (marked by arrows) gives an experimental measurement of the pairing gap [213].

in ${ }^{194} \mathrm{~Pb}$ panel (b) and ${ }^{192} \mathrm{~Pb}$ panel (c). These two SD bands decay out at different spins, namely $6 \hbar$ in the case of ${ }^{194} \mathrm{~Pb}$ and $10 \hbar$ for ${ }^{192} \mathrm{~Pb}$. The expected endpoints of the decay-out spectra (obtained via a QC analysis for ${ }^{192} \mathrm{~Pb}$ and from single-step links as well as a $\mathrm{QC}$ analysis for ${ }^{194} \mathrm{~Pb}$ ) are indicated with arrows in Fig. $44(\mathrm{U}(6 \hbar)$ and $\mathrm{U}(10 \hbar)$ respectively) while the onset of the QC spectrum is shown with a dotted line. One can clearly see from the figure that the pairing gap in the decay-out spectrum decreases with spin, from $0.95 \mathrm{MeV}$ at $6 \hbar$ in ${ }^{194} \mathrm{~Pb}$ to $0.4 \mathrm{MeV}$ at $10 \hbar$ for ${ }^{192} \mathrm{~Pb}$. So as expected pairing is reduced in the ND well with increasing spin.

A way to determine the excitation energy and spin of an SD band is by analysing the shape and intensity of the decay-out $\mathrm{QC} \gamma$ spectrum. This technique is called $\mathrm{QC}$ analysis. The results from the QC analysis are an important complement in cases where only one or two direct linking transitions are observed. In many cases though, one-step linking transitions are not observed and the QC analysis may be the only option available.

The area of the decay-out spectrum is normalized so that its integral represents the average multiplicity $\langle M\rangle$ of the decay-out cascade. From the shape of the spectrum, the average $\gamma$-ray energy $\left\langle E_{\gamma}\right\rangle$ can be found and by multiplying the two one can get the average energy $E_{x}$ removed by the decay-out QC:

$$
E_{x}=\left\langle E_{\gamma}\right\rangle\langle M\rangle
$$

Assuming that each decay-out transition removes $\left\langle\delta I_{\gamma}\right\rangle=0.5(1) \hbar$ (this number follows from Monte Carlo simulations $[15,141]$ of a statistical decay), one can compute the average spin $I_{x}$ removed by the 
decay-out cascades:

$$
I_{x}=\left\langle\delta I_{\gamma}\right\rangle\langle M\rangle
$$

From the intensities of the ND states populated by the decay-out cascade, one can find the average entry point into the ND yrast line. Adding the $E_{x}$ and $I_{x}$ removed by the decay-out $\gamma$-rays to this ND entry point, one can find the average SD exit point out of the SD yrast line. These exit and entry points are shown in the cartoon of Fig. 6.
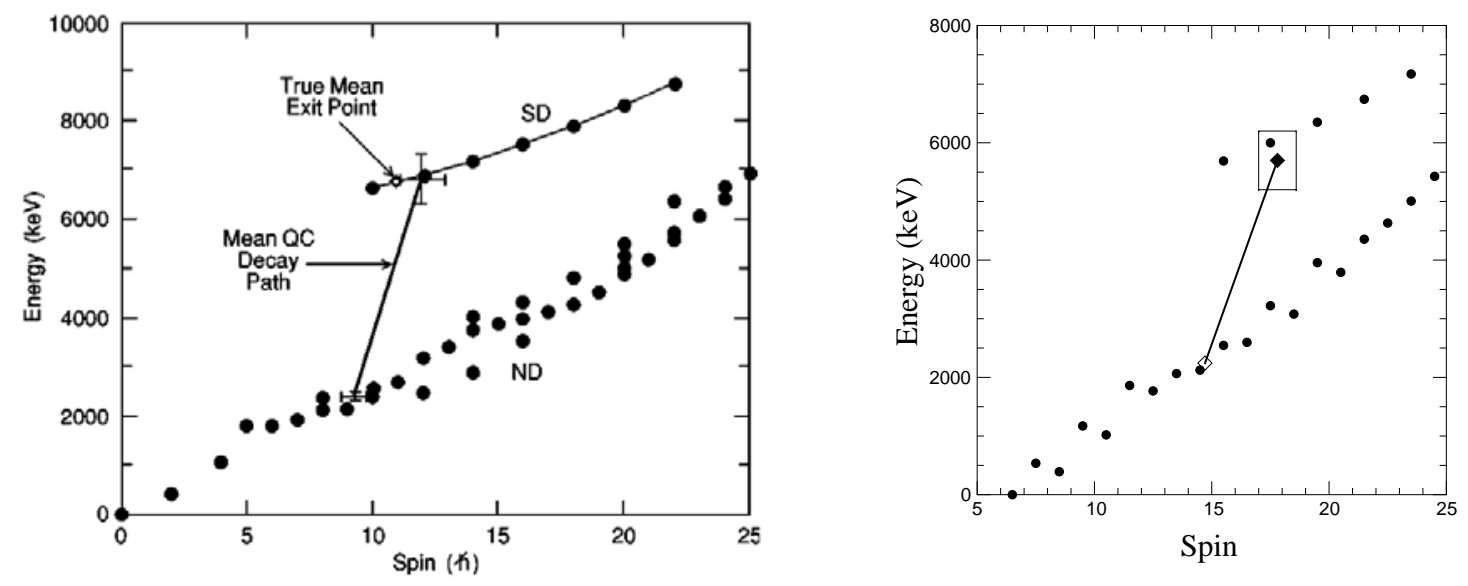

Figure 45: The experimental ND and SD yrast lines in ${ }^{194} \mathrm{Hg}$ (left) and ${ }^{191} \mathrm{Hg}$ (right) are shown by the filled circles. The SD exit points obtained from the QC analysis are shown by the data point with error bars in ${ }^{194} \mathrm{Hg}$ and with a filled diamond in a error box in the case of ${ }^{191} \mathrm{Hg}$ [15, 156].

The case of ${ }^{194} \mathrm{Hg}$, where the (I,E) ${ }_{S D}$ of the yrast SD band is known from several one step linking transitions, was used to test how well the QC analysis method works [15]. This was also checked for ${ }^{194} \mathrm{~Pb}$ [213] and later also in the odd case of ${ }^{191} \mathrm{Hg}$ [156]. The results of the QC analysis for the yrast $\mathrm{SD}$ band in ${ }^{194} \mathrm{Hg}$ give a mean SD exit point of $11.9(1.0) \hbar$ and $6.8(5) \mathrm{MeV}$. In Fig. 45 (left panel) the results from the QC analysis are compared to the true mean SD exit point $(11.1 \hbar$ and $6.77 \mathrm{MeV})$ deduced from the intensity of the SD band transitions. For the case of ${ }^{194} \mathrm{~Pb}$ the QC analysis gives the excitation energy of the SD yrast band above the ND yrast at $6 \hbar, \mathrm{U}(6 \hbar)$, of $2.7(2) \mathrm{MeV}$ compared to $2.743 \mathrm{MeV}$ extracted from linking transitions $[94,195]$. The agreement in both cases is very good. The results from the QC analysis of the decay-out QC from the yrast SD band of ${ }^{191} \mathrm{Hg}$ is shown in Fig. 45 (right panel). The filled diamond gives the average SD exit point obtained from the QC analysis. The box around the filled diamond shows the uncertainty in spin and energy from the QC analysis. Again the results from the $\mathrm{QC}$ analysis are in good agreement (within the error bars) with the results from the one-step linking transitions. In the case of ${ }^{192} \mathrm{~Pb}$ [213], the results obtained from the QC analysis $(\mathrm{U}(10 \hbar)=2.1(3) \mathrm{MeV})$ were in perfect agreement (within the error bars) with the value extracted 3 years later from linking transitions (2.079 MeV) [186]. This gives confidence about the spin and energy assignments from QC analysis in cases where there are no one-step linking transitions, like ${ }^{192} \mathrm{Hg}$.

An alternative method to determine the excitation energy and spin of SD states consists in analysing the entry distribution feeding SD states (cf Sec. 2.2). The lower limit of this entry distribution provides an estimate of the actual SD yrast line. Johnson et al. [78] have extracted the (I,E) entry distribution for populating SD states in ${ }^{198}$ Po. The lower limit of this distribution at $21 \hbar$ was found to be $6.2(5)$ $\mathrm{MeV}$. Extrapolating to 0 spin using the yrast SD band moment of inertia, the excitation of SD states in ${ }^{198} \mathrm{Po}$ was found to be $3.9(4) \mathrm{MeV}$ above the ground state. This is in good agreement with earlier predictions by Krieger and coworkers [218]. 
Another slightly different method to extract information from the (I,E) entry distribution is given in Ref. [141]. The entry distributions leading to ND and SD states in ${ }^{192} \mathrm{Hg}$ was measured. Then the model based on Monte Carlo simulations of $\gamma$ cascades presented in Sec. 4.1.1, was used to successfully reproduce all the known observables connected with the population of SD states. Comparison of experimental and model results, together with the measured SD entry distribution, suggest that the SD band lies 3.3-4.3 MeV above the normal yrast line when it decays around spin 10ћ. This, again, is in good agreement with what was found by a QC analysis of ${ }^{192} \mathrm{Hg}[122,15]$.

In the cases of $\mathrm{Hg}$ and $\mathrm{Pb}$ where one has a chain of isotopes (or isotones) where the excitation energy and spin of the SD band has been determined (either via single-step links or a QC analysis), it becomes interesting and meaningful to compare with theoretical calculations. In order to compare the experimental data with theory, the excitation energies of the SD band has been extrapolated down to the the bandhead of the yrast $\mathrm{SD}$ bands $(\mathrm{I}=0)$ in the even-even $\mathrm{Hg}$ and $\mathrm{Pb}$ isotopes. For the odd $\mathrm{A}$ $\left({ }^{191} \mathrm{Hg}\right)$ the SD band is extrapolated to zero rotational frequency (corresponding to $\left.\mathrm{I}=2.9 \hbar\right)$ using the Harris expansion [219] for the dynamic moment of inertia.
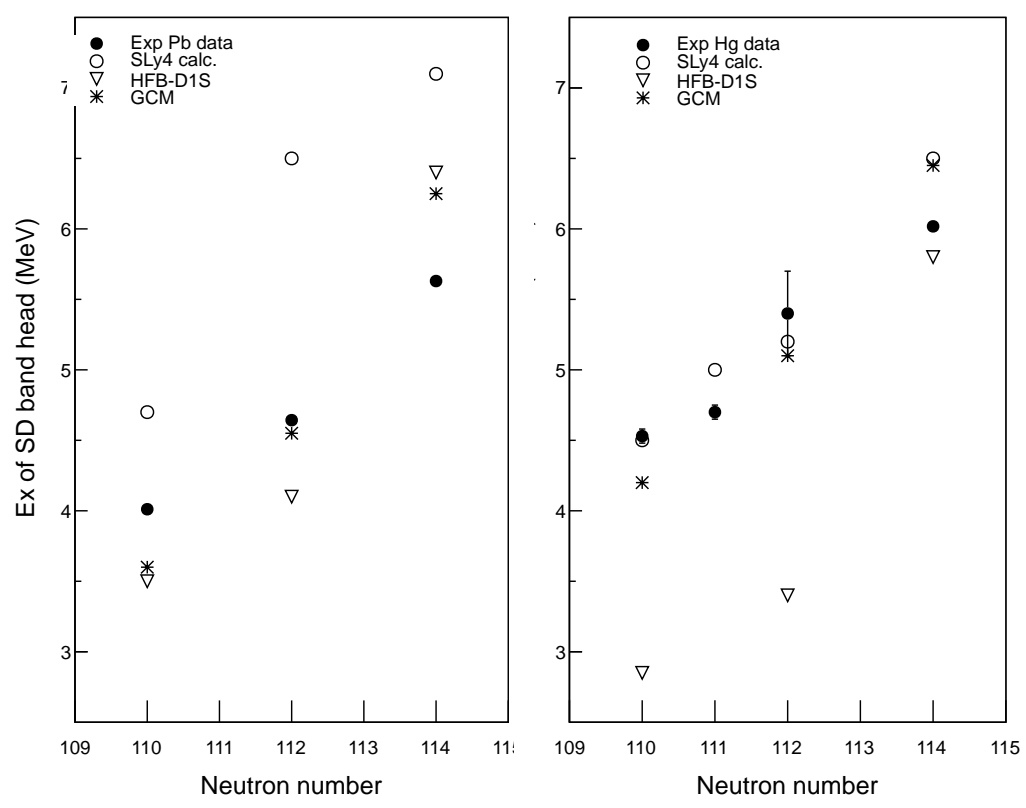

Figure 46: Experimental SD bandhead energies for $\mathrm{Hg}$ (right panel) and Pb nuclei (left panel), compared with the results of static (SLy4 Skyrme [220] and D1S Gogny [109] ) and dynamic (GCM [109]) meanfield calculations $[188,15,156]$.

In Fig. 46 the experimental SD bandhead energies (full circles) are compared to existing theoretical predictions for $\mathrm{Hg}$ and $\mathrm{Pb}$ isotopes (open symbols and star). The results of three theoretical approaches are shown: static mean-field (Hartree-Fock-Bogolyubov) calculations employing state-ofthe-art parametrizations of the Skyrme (SLy4) and Gogny (D1S) interactions [220, 109], and generator coordinate method (GCM) calculations [109] (also using the Gogny D1S interaction). The parameters of the effective interactions in these calculations are obtained from fits to bulk properties of normal (closed-shell) nuclei. Predictions of SD bandhead energies then represent good tests of theory in a new domain. The main difference between the HFB and GCM approaches is that the latter takes into account long-range correlations and thus allows for the effects of quadrupole vibrational modes. Although the static mean-field calculations employing the Skyrme (Gogny) interaction reproduce the Hg energies 
well, it is only the calculations including dynamic shape fluctuations (beyond mean field with the GCM) that simultaneously reproduce the excitation energies in both isotopic chains to within $0.5 \mathrm{MeV}$.

\subsubsection{Pairing properties of superdeformed nuclei}

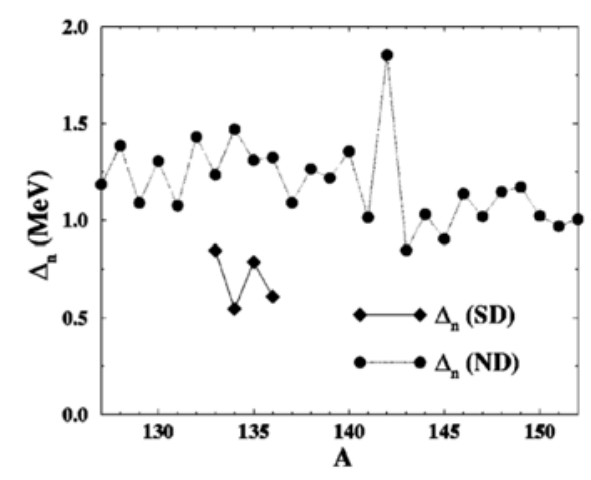

Figure 47: Experimental neutron pairing gaps for ${ }^{133-136} \mathrm{Nd}$ at $\mathrm{SD}$ shapes and for ${ }^{127-152} \mathrm{Nd}$ in their ND ground states. SD points correspond to the mean values of $\Delta_{n}$ extracted within the spin range of the SD bands [183].

For Neodynium the excitation energies of SD bands have been determined for six isotopes via onestep linking transitions. From these data it was possible to extract the neutron pairing gap in the SD well of $\mathrm{Nd}$ nuclei using a 3-point mass formula. In the independent quasi-particle picture, and with some assumptions on the contribution of rotation to the excitation energy of the six SD nuclei, one can relate the odd-even mass difference at spin I to an average neutron pairing gap $\Delta_{n}$ [183]. The results for the Nd isotopes, are shown in Fig. 47 for both ND and SD shapes. The most striking feature of the results is that the pairing gap for the SD shapes is reduced by a factor of 2 with respect to the ND value $\left(\Delta_{n}(\mathrm{SD}) \approx 0.7 \mathrm{MeV}\right)$. The values of $\Delta_{n}$ for SD matter are mean values extracted within the spin range of the bands. The error bars are large $(\sim 50 \%)$ due to the approximations made and the uncertainties in the mass values but it is found that pairing - although reduced - is still present in mass 130 SD nuclei and at relatively high spins [183].

In the cases where the decay-out spectrum has been isolated from the rest of the $\gamma$-rays emitted by the nucleus, important information was obtained on the nature of the coupling between SD and ND states as well as on the properties of the spectrum of ND states sampled by the nucleus. The chaoticity of ND states has been addressed in the neutron resonance region [30], in the case of nuclear masses [221], of near yrast states [222] and of excited states at low spin [223]. The decay from SD states provides a new region in excitation and spin to the study the chaoticity of ND states: at 0 spin in the fission isomers, at moderate spins in the mass 190 region and at higher spin in the mass 150 region. Finally, in a handful of cases, the decay-out spectrum has revealed essential spectroscopic observables such as $E^{*}$, spin and parity for SD states. More data is required to test and constrain theoretical models, in particular odd nuclei. 


\section{Conclusion and Outlook}

\subsection{Conclusion}

By analysing the complete $\gamma$-ray spectra coincident with SD lines, it has been possible to unravel the separate components emitted from each stage of the electromagnetic cascade from beginning to end, i.e. from the entry distribution (after particle emission) to the ground state. The results reveal a fascinating adventure when the flow goes through the SD well, revealing the feeding and decay mechanisms of SD bands and a unique double cycle of chaos, order, chaos and order, instead of the usual single cycle. The population mechanism is mainly a statistical process, which takes into account the coupling between hot ND and SD states. Spectral features (such as SD band intensity, including the variation with spin, as well as 1- and 2D E2 QC shapes) can be described in simulations, one using only statistical features based on level densities and $\gamma$ strength functions, another that includes microscopically calculated states (and can also account for the number of rotational pathways). The comparison of experimental and simulated spectra also give insight into the structure of excited SD bands, while also testing and validating theoretical predictions of their microscopic character. In the mass 190 region, specifically in

${ }^{194} \mathrm{Hg}$, a new phenomenon has been observed in the transition from chaos to order, where an intermediate region of ergodic bands emerges. These bands are characterised by collective flow channeled along nearly parallel bands, despite complicated, nearly-chaotic wave functions. At the onset of chaotic mixing in all other cases, including for ND nuclei, the E2 flow is highly fragmented over a 30-fold broader energy interval. Theory predicts the ergodic bands and provide insights into their origin. The small energy (frequency) dispersion in the narrow rotational ridges is due to quasiparticle states of high $\mathrm{K}$ with small alignment $i$ and also small dispersion $\Delta i$, abetted by a large moment of inertia due to large deformation and mass: $\Delta \omega=\Delta i / \Im^{(2)}$. The identical $\Im^{(2)}$ detected in SD bands in this mass region (and also in actinide nuclei) can be traced to the very same conditions, so that the two phenomena are directly connected.

The decay from the SD minimum to excited ND states is now well understood in terms of experiment and theory. The cold SD state couples weakly with a nearby ND state (or states), thereby acquiring a small ND admixture in its wave function and tunnels across the separating barrier. Quantum mechanics provides a simple solution to the mystery of how the nucleus undergoes a major rearrangement of shape and wave function: $\gamma$ or particle decay occurs via the admixed ND component. When the ND state is highly excited (as in the mass 150 and 190 regions), the ND state is chaotic, which enhances the tunnelling, i.e. it is chaos-assisted tunnelling. From a nuclear physics perspective, one can understand that coupling to a compound state is not suppressed by selection rules, which would otherwise hinder the coupling to a state with different, conserved quantum numbers. Analogies from chaos science provide a compact language and insight to describe the decay process. The decay spectrum out of the SD minimum is remarkably identical to that from neutron capture. Theory can describe the main features of this spectrum, although there are differences. These can be attributed to approximations in the level density and reduced pairing of hot ND states, suggesting that the decay spectrum can provide information on both these properties.

The measurement of the energies of SD states has been a challenge for experiment, due to the small SD population and even weaker intensity of the high-energy primary transitions (governed by PorterThomas fluctuations) decaying directly to near-yrast ND states. Nevertheless, in a few cases, the energies have been measured. These are important tests for theoretical models, where parameters are fixed based on properties of ND nuclei, usually magic nuclei. Predictions on SD properties represent true predictions because SD states are in a completely different regime (in a similar fashion as superheavy elements). It turns out that it is a challenge for theory to accurately describe the energies and mass trends of SD states to better than $\pm 0.5 \mathrm{MeV}$. 


\subsection{Outlook}

So far, QC studies of SD nuclei have been limited to the heavier mass regions. Moreover, the studies have only been performed in coincidence with ground state SD bands. In the future, one could extend the investigations to lighter nuclei and to excited SD bands, to investigate the influence of the mass number, K-quantum number and reduced pairing.

Computing the decay-out spectra with realistic pairing, level densities and excitation energies would test model predictions of level densities and the reduction of pairing with excitation energy. In general, it has proven a challenge for theory to accurately predict the excitation energy of SD states. Systematic determination of $I, \pi$ and experimental excitation energies, will provide solid tests of theory. In addition, energies and, hence masses, of SD states along an isotopic chain will provide a quantitative measure of pairing at large deformation in the SD well, e.g. from 3- or 5-point mass formulae.

The role of quantum numbers in tunnelling is a subject of general interest. Questions to explore are, e.g., are they conserved and for how long in the tunnelling process? Efforts to investigate the decay out properties for bands of different $\mathrm{K}$ will provide information on this subject (along with complementary studies of the role of $\mathrm{K}$ in fission).

The ergodic bands in ${ }^{194} \mathrm{Hg}$ are unique in nuclear physics. How widespread are they among SD bands ? Theory predicts that they arise out of shell structure, should occur in other cases of the mass 190 region, and should have systematic variations with $\mathrm{Z}$ and $\mathrm{N}$, as the configurations around the Fermi level change. This is an opportunity for nuclear physics to contribute to an understanding of the order-to-chaos transition.

The theoretical, simulation and data-analysis tools as well as detectors have considerably evolved since the early days of SD and there are many possibilities of looking at old results with a new eye and to analyse new and better data. As an example, one can mention:

(i) The side-feeding lifetimes, which come out from SD line-shape analyses using the Doppler Shift Attenuation Method, are naturally computed by the decay-flow simulations codes presented in this review. As discussed in Sec. 3.5, the comparison between the experimental and simulated values would be highly instructive and could shed light on the mixing of SD and ND states at energies above the barrier, that is how rapidly does the barrier with increasing excitation energy loose its ability to separate the states at the different deformations.

(ii) Studying the ridges and feeding QC as a function of spin, by gating on specific SD transitions to force the decay flow to reach the yrast line at a given spin.

(iii) The possibility of an inverted analysis by gating on SD ridges to obtain one-dimensional spectra. This way, one could observe the feeding patterns into both the SD and ND yrast lines and deduce important information on the trapping probability in the second well as well as its evolution with spin. (iv) The techniques developed to study SD QC spectra can be used in other fields. As an example, the search for weak regular signals, such as hyperdeformed rotational bands or ridges, could benefit from tilted rotational planes. Attempts in this direction were performed with EUROBALL [224] and GAMMASPHERE in the mass 130 region.

(v) The knowledge acquired on how to go about decomposing spectra into various components will also be helpful to look into the presence (or not) of an M1 scissors resonance in transfermium nuclei, for which such a mode has been predicted [225] .

(vi) More advanced simulations including the competition with M1 transitions could be helpful in treating near-yrast cascades but also the decay of hotter states in the presence of enhanced M1 strength. (vii) The new generation of tracking arrays, with their enhanced efficiency, especially at high energy, will help address the still open issue regarding the enhanced cooling of SD states via the lower-energy component of the GDR. Better efficiency should provide the essential quantum numbers $(E, I, \pi)$ of more SD bands and enable investigations of high-K isomers in the second well. 


\subsection{Next generation of arrays and accelerator facilities}

The next generation of $\gamma$-ray multi-detector arrays such as AGATA [57] in Europe and GRETINA [56] in the USA will bring new perspectives into the study of high spin phenomena and the associated quasi-continuous spectra. It will be a challenge to transpose the methods developed for the standard Compton-suppressed arrays, in particular the applicability of certain techniques, such as removing Compton background, correcting for photon pileup and multi-dimensional background subtraction, needs careful checking. The advantage of AGATA and GRETINA, on top of their efficiency and Doppler-correction capabilities, is the fact that the polar angle of the emitted $\gamma$-rays with respect to the beam axis can be measured continuously and this also is the case for the linear polarisation of the Compton-scattered photons. The decomposition of spectra in terms of quadrupole and dipole component, as well as electric and magnetic types of radiation, will no doubt benefit from the highprecision angular information as well as the innate polarisation sensitivity. These properties will be very helpful in disentangling overlapping QC components. Moreover, the availability of high intensity radioactive beams will make it possible to perform, in the coming years, similar types of studies as those presented in this review in exotic regions of the nuclear chart. QC spectroscopy might turn out to be, in some cases, the only way to observe weakly populated rotational structures, similarly to the earlier discovery of superdeformation.

\section{Acknowledgements}

This material is based upon work supported by the U.S. Department of Energy, Office of Science, Office of Nuclear Physics, under contract number DE-AC02-06CH11357. This research used resources of the ANL/ATLAS facility, which is a DOE Office of Science User Facility. Support from the CNRS/IN2P3, INFN, NBI and the Norwegian Research Council is also acknowledged.

\section{References}

[1] V. M. Strutinsky. Nucl. Phys. A, 95:420, 1967.

[2] S. Hofmann and G. Münzenberg. Rev. Mod. Phys., 72:733, Jul 2000.

[3] K. Morita et al. Journal of the Physical Society of Japan, 81(10):103201, 2012.

[4] Yu. Ts. Oganessian et al. Phys. Rev. C, 74:044602, 2006.

[5] S. M. Polikanov et al. Zh. Eksp. Teor. Fiz., 42:1016, 1962.

[6] S. Bjørnholm and J. E. Lynn. Rev. Mod. Phys., 52:725, 1980.

[7] P. Twin et al. Phys. Rev. Lett., 57:1380, 1986.

[8] P. J. Nolan and P. J. Twin. Annu. Rev. Nucl. Part. Sci., 38:533, 1988.

[9] R. V. F. Janssens and T. L. Khoo. Annu. Rev. Nucl. Part. Sci., 41:321, 1991.

[10] A. Bohr and B. R. Mottelson. Nuclear Structure Vol 2. Benjamin, New York, 1969.

[11] M. Birch and B. Singh. priv. comm., 2015.

[12] B. Singh et al. Nuclear Data Sheets, 97:241, 2002. 
[13] B. Singh. priv. comm., 2015.

[14] E. F. Moore et al. Phys. Rev., C55:R2150, 1997.

[15] T. Lauritsen et al. Phys. Rev. C, 62:044316, 2000.

[16] P. Fallon et al. Phys. Rev. C, 60:044301, Aug 1999.

[17] G. Hackman et al. Phys. Rev. Lett., 79:4100-4103, 1997.

[18] I. Ahmad et al. Phys. Rev. C, 44:1204-1207, Sep 1991.

[19] S. Flibotte et al. Phys. Rev. Lett., 71:4299-4302, Dec 1993.

[20] D.S. Haslip et al. Phys. Rev. Lett., 78:3447-3450, May 1997.

[21] I. Hamamoto and B. R. Mottelson. Phys. Lett. B, 333:294, 1994.

[22] F. Donau et al. Phys. Lett. B, 387:667, 1996.

[23] S.W. Ødegard et al. Phys. Rev. Lett., 86:5866, 2001.

[24] G. Schönwasser et al. Phys. Lett.B., 552:9, 2003.

[25] H. Amro et al. Phys. Lett. B, 553:197, 2003.

[26] S.W. Ødegaard et al. Phys. Rev. Lett., 86:5866-5869, Jun 2001.

[27] D. Rudolph et al. Phys. Rev. lett, 80:3018, 1998.

[28] D. Rudolph et al. Phys. Rev. Lett., 86:1450, 2001.

[29] B. Nyako et al. Phys. Rev. Lett., 52:507, 1984.

[30] R. U. Haq et al. Phys. Rev. Lett., 48:1086-1089, Apr 1982.

[31] O. Bohigas et al. Phys. Rev. Lett., 52:1-4, Jan 1984.

[32] B. R. Mottelson. Proceedings of the International Seminar on the Frontier of Nuclear Spectroscopy. World Scientific, Singapore, 1993.

[33] F. Soramel et al. to be published.

[34] T. L. Khoo. Proceedings from the institute of nuclear theory, vol. 5, tunneling in complex systems. page 229. World Scientific, 1998.

[35] Proceedings from the institute of nuclear theory, vol. 5, tunneling in complex systems. World Scientific, 1998.

[36] O. Bohigas et al. Nucl. Phys. A, 560:197, 1993.

[37] S. Tomsovic et al. Phys. Rev. E, 50:145-162, Jul 1994.

[38] S. AAberg. Phys. Rev. Lett., 82:299, 1999.

[39] P. Twin et al. Workshop on nuclear structure at high spins, risø, denmark. page 135, 1981.

[40] C.Rossi Alvarez. NIM, A359:500, 1995. 
[41] R. Diamond. Instr. for heavy ion nucl. res. In Nucl. Sci. Research Conf. Series, volume 7, page 259 .

[42] J.P. Martin et al. Nucl. Instr. Meth. A, 257:301, 1987.

[43] R. Leider et al. Nucl. instr. Meth., 220:363, 1984.

[44] B. Hersind et al. Nucl. Phys. A, 447:395c, 1985.

[45] F. Beck et al. Instr. for heavy ion nucl. res. In Nucl. Sci. Research Conf. Series, volume 7, page 129.

[46] P. J. Nolan et al. Annu. Rev. Nucl. Sci., 45:561, 1994.

[47] J. Eberth and J. Simpson. Progress in Particle and Nuclear Physics, 60:283, 2008.

[48] R. Holzmann et al. Phys. Rev. Lett., 62:520, 1989.

[49] R. Holzmann et al. Phys. Lett., B195:321, 1987.

[50] D. C. Radford et al. Nucl. Instrum. Methods, A258:111, 1987.

[51] R. Holzmann et al. Nucl. Instrum. Methods., A260:153, 1987.

[52] J. Simpson. Z. Phys., A358:139, 1997.

[53] I-Y. Lee. Nucl. Phys., A520:641c, 1990.

[54] G. J. Schmid et al. NIM, A 430:69, 1999.

[55] J. Van der Marel and B. Cederwall. Nucl. Instr. Meth. A, 487:441, 1999.

[56] S. Paschalis et al. Nucl. Instrum. Methods A, 709(0):44 - 55, 2013.

[57] S. Akkoyun et al. Nucl. Instrum. Methods A, 668(0):26 - 58, 2012.

[58] I-Yang Lee and J. Simpson. Nulcear Physics News, 20:23, 2010.

[59] T. Lauritsen et al. Phys. Rev., C62:044316, 2007.

[60] T. Lauritsen et al. Phys. Rev. C, 62:044316, 2000.

[61] V. Martin, J. L. Egido, T. L. Khoo, and T. Lauritsen. Phys.Rev., C51:3096, 1995.

[62] D. C. Radford. http://radware.phy.ornl.gov, 2004.

[63] R. M. Lieder and H. Ryde. Adv. Nucl. Phys., 10:1, 1978.

[64] S. J. Feenstra. Thesis, Rijksuniversiteit Groningen, The Netherlands, 1979.

[65] R. M. Diamond and F. S. Stephens. Ann. Rev. Nucl. Part. Sci., 30:85, 1980.

[66] O. Andersen et al. Phys. Rev. Lett., 43:687, 1979.

[67] S. W. Odegard et al. Eur.Phys.J. A, 14:309, 2002.

[68] T. Døssing et al. Phys. Rep., 268:1, 1996. 
[69] S. Leoni et al. Eur. Phys J., A 4:229, 1999.

[70] A. Lopez-Martens et al. Phys.Rev.Lett., 100:102501, 2008.

[71] B. Herskind et al. Phys.Rev.Lett., 68:3008, 1992.

[72] S. Leoni et al. Phys. Lett. B, 353:179, 1995.

[73] S. Leoni et al. Phys. Rev. Lett., 101:142502, 2008.

[74] B. Herskind et al. Phys. Scr. T, 125:108, 2006.

[75] S. Leoni et al. Phys. Rev. C, 79:064306, 2009.

[76] B. Crowell et al. Nucl. Instrum. Methods., A355:575, 1995.

[77] Ph. Benet. Ph.D. Thesis. CRN Strasbourg, CRN/PN88-29, 1988.

[78] M. S. Johnson et al. Phys.Rev. C, 71:024317, 2005.

[79] P. Reiter et al. Phys. Rev. Lett., 84:3542, 2000.

[80] G. Henning et al. Phys. Rev. Lett., 113:262505, 2014.

[81] A. Lopez-Martens et al. Phys. Rev. Lett., 77:1707, 1996.

[82] A. Bracco et al. Rep. Prog. Phys., 65:299, 2002.

[83] S. Frattini et al. Phys. Rev. Lett., 81:2659, 1998.

[84] S. Frattini et al. Phys. Rev. Lett., 83:5234, 1999.

[85] S. Leoni et al. Nuc. Phys. A, 671:71, 2000.

[86] P. Bosetti et al. Phys. Rev. Lett., 76:1204, 1996.

[87] G. Benzoni et al. Phys. Lett. B, 615:160, 2005.

[88] S. Leoni et al. Phys. Rev. C, 72:034307, 2005.

[89] V. Vandone et al. Phys. Rev. C, 88:034312, 2013.

[90] M. Matsuo et al. Nuc. Phys. A, 736:223, 2004.

[91] S. Leoni et al. Phys. Lett. B, 409:71, 1997.

[92] S. Leoni et al. Phys. Lett. B, 498:137, 2001.

[93] S. Leoni et al. Phys. Rev. C, 79:064307, 2009.

[94] A. Lopez-Martens et al. Phys. Lett., B380:18, 1996.

[95] T. Døssing et al. Phys. Rev. Lett., 75:1276, 1995.

[96] B. R. Mottelson. Nucl. Phy., A557:717c, 1993.

[97] K. Yoshida et al. Nuc. Phys. A, 696:85, 2001. 
[98] M. Brack et al. Rev. Mod. Phys, 44:320, 1972.

[99] K. Neegaard et al. Nucl. Phys. A, 262:61, 1976.

[100] I. Ragnarsson et al. Nucl. Phys. A, 347:287, 1980.

[101] G. G. Andersson et al. Physica Scripta, 24:266, 1981.

[102] A. Bohr and B. R. Mottelson. Nuclear Structure, volume II. Benjamin, 1975.

[103] A. G. Magner et al. Phys. Rev. E, 63:065201, 2001.

[104] J. Dudek et al. Phys. Rev. C, 38:940, 1988.

[105] Y. R. Shimizu et al. Nucl. Phys. A, 557:c99, 1993.

[106] D.L. Hill and J.E Wheeler. Phys. Rev., 89:1102, 1953.

[107] P. Bonche et al. Nucl. Phys A, 519:509, 1990.

[108] J. Meyer et al. Nucl. Phys. A, 588:597, 1995.

[109] J. Libert et al. Phys. Rev. C, 60:054301, 1999.

[110] J. P. Delaroche et al. Phys. Rev. C, 81(014303), 2010.

[111] K.Schiffer and B.Herskind. Phys. Lett. B, 255:508, 1991.

[112] K. Schiffer and B. Herskind. Nucl. Phys A, 520:C521, 1990.

[113] K. Schiffer et al. Z. Phys. A, 332:17, 1989.

[114] H. A. Weidenmüller et al. Phys. Rev. Lett., 81:3603, 1998.

[115] G. F. Auchampaugh and L. W Weston. Phys. Rev. C, 12:1850, 1975.

[116] A.C. Larsen et al. Phys. Rev. C, 83:034315, 2011.

[117] A. Bohr and B. R. Mottelson. Nuclear Structure, volume I. Benjamin, 1969.

[118] S. Aberg. Nucl. Phys., A477:18, 1988.

[119] E. Vigezzi et al. Phys. Lett. B, 249:163, 1990.

[120] D. M. Cardamone et al. Phys. Rev. Lett., 91:102502, 2003.

[121] D. Curien et al. Phys. Rev. Lett., 71:2559, 1993.

[122] R. G. Henry et al. Phys. Rev. Lett., 73:777, 1994.

[123] J. Gu and H. A. Weidenmüller. Nucl. Phys., A660:197, 1999.

[124] C.A. Stafford and B.R. Barrett. Phys. Rev. C, 60:051305, 1999.

[125] R. Kruecken et al. Phys. Rev. C, 55:064316, 1997.

[126] R. Kruecken et al. Phys. Rev. C, 64:064316, 2001. 
[127] D. M. Cardamone et al. Phys. Lett. B, 661:233, 2008.

[128] B. R. Barrett et al. Phys. Lett. B, 688:110, 2010.

[129] C. Andreoiu et al. Phys. Rev. Lett., 91:232502, 2003.

[130] M.A. Deleplanque et al. Phys. Rev. Lett., 40:629, 1978.

[131] G. Leander. Phys. Rev., C25:2780, 1982.

[132] B. Lauritzen et al. Nuc. Phys. A, 457:61, 1986.

[133] M. Matsuo, T. Døssing, B. Herskind, S. Frauendorf, E. Vigezzi, and R. A. Broglia. Nucl. Phys., A557:211c, 1993.

[134] M. Matsuo et al. Nuc. Phys. A, 617:1, 1997.

[135] R. A. Broglia, T. Døssing, B. Lauritzen, and B. R. Mottelson. Phys. Rev. Lett., 58:326, 1987.

[136] M. Matsuo, T. Døssing, E. Vigezzi, and R. A. Broglia. Phys. Lett., B465:1, 1999.

[137] F. S. Stephens et al. Phys. Rev. Lett., 88:142501, 2002.

[138] S. Leoni et al. Phys. Rev. Lett., 93:022501, 2004.

[139] F. S. Stephens et al. Phys.Rev. C, 78:034303, 2008.

[140] A. Lopez-Martens et al. Nucl. Phys. A, 647:217, 1999.

[141] T. Lauritsen et al. Phys. Lett., B279:239, 1992.

[142] G. A. Leander. Computer Phy. Com., 47:311, 1987.

[143] A. Bracco et al. Phys. Rev. Lett., 76:4484, 1996.

[144] K. Yoshida et al. Nuc. Phys. A, 612:26, 1997.

[145] K. Yoshida and M. Matsuo. Nucl. Phys., A636:169, 1998.

[146] A. Winther. Nuc. Phys. A, 594:203, 1995.

[147] F. Pulhofer. Nuc. Phys. A, 240:267, 1977.

[148] S. Aberg. Nuc. Phys. A, 477:18, 1988.

[149] P.F. Bortignon et al. Giant resonances: Nuclear structure at finte temperature. Harwood, New York, 1998.

[150] M. Gallardo et al. Nuc. Phys. A, 443:415, 1985.

[151] K. E. G. Lobner. Phys. Lett. B, 26:369, 1968.

[152] T. Lauritsen et al. Phys. Rev. Lett., 88:042501, 2002.

[153] D. Rosbach et al. Phys. Lett. B, 513:9, 2001.

[154] A. Korichi et al. Phys. Rev. Lett., 86:2746, 2001. 
[155] D.C. Radford et al. Nucl. Instrum. Methods A, 361:297, 1995.

[156] S.Siem et al. Phys. Rev., C70:014303-1, 2004.

[157] R. V.F. Janssens et al. Phys. Lett., B152:167, 1985.

[158] J. P. Thibaud et al. Zeitscrift fur Physic, A321:275, 1985.

[159] J. O. Newton and G. D. Dracoulis. Phys. Rev. Lett., 40:625, 1978.

[160] B. Herskind et al. Phys. Rev. Lett., 59:2416, 1987.

[161] A. Bracco et al. Nuc. Phys. A, 682:449c, 2001.

[162] A. Maj et al. Nuc. Phys. A, 571:185, 1994.

[163] S. Leoni et al. Phys. Rev. Lett., 76:3281, 1996.

[164] P. Taras et al. Phys. Rev. Lett., 61:1348, 1988.

[165] G. Benzoni et al. Phys. Lett. B, 540:199, 2002.

[166] M. Kicinska-Habior et al. Phys. Lett. B, 308:225, 1993.

[167] A: Maj et al. Nuc. Phys. A, 687:192, 2001.

[168] A. Maj et al. Nuc. Phys. A, 731:319, 2004.

[169] M. Kmiecik et al. Acta Phys. Pol. B, 36:1169, 2005.

[170] W.J. Swiatecki W.D. Myers. Acta Phys. Pol. B, 32:1033, 2001.

[171] K. Pomorski and J. Dudek. Phys. Rev. C, 67(044316), 2003.

[172] A. Gadea et al., 1999.

[173] M. Brekiesz et al. Acta Phys. Pol. B, 36:1175, 2005.

[174] F. Farget et al. Phys. Rev. C, 58:150, 1998.

[175] B. Million et al. Phys. Lett. B, 415:321, 1997.

[176] M.A. Deleplanque et al. Phys. Rev. Lett., 41:1105, 1978.

[177] D. Ward et al. Phys. Rev. C, 66:024317, 2002.

[178] F. S. Stephens et al. Phys. Rev. Lett., 94:042501, 2005.

[179] D. Bazzacco et al. Phys. Rev. C, 49:R2281, 1994.

[180] C. Petrache et al. Phys. Lett. B, 415:223, 1997.

[181] C. Petrache et al. Phys. Rev. Lett., 77:239, 1996.

[182] M.A. Deleplanque et al. Phys. Rev. C, 52:2302, 1995.

[183] S. Perries et al. Phys. Rev. C, 60:064313, 1999. 
[184] S. Lunardi et al. Phys. Rev. C, 52:6, 1995.

[185] T. L. Khoo et al. Phys. Rev. Lett., 76:1583, 1996.

[186] A. N. Wilson et al. Phys. Rev. Lett., 90:142501-1, 2003.

[187] A. Wilson et al. Phys. Rev. Lett., 95:182501, 2005.

[188] A. Wilson et al. Phys. Rev. Lett., 104:162501, 2010.

[189] S. Bounneau et al. Eur. Phys. J. A, 2:245, 1998.

[190] R. Krücken et al. Eur. Phys. A, 5:367, 1999.

[191] A. Ataç et al. Phsy. Rev. Lett., 70:1069, 1993.

[192] F. Lerma et al. Phys. rev. C, 56:R1671, 1997.

[193] J. Kantele et al. Phys. Rev. C, page 1693, 1984.

[194] J. Schirmer et al. Phys. Lett., 63:2196, 1989.

[195] K. Hauschild et al. Phys. Rev., C55:2819, 1997.

[196] Ch. Chiara et al. Phys. Rev. C, 73:021301R, 2006.

[197] C. Svensson et al. Phys. Rev. Lett., 82:3400, 1999.

[198] D. Rudolph et al. Phys. Rev. C, 63:021301, 2001.

[199] J. Domscheit et al. Nucl. Phys. A, 660:381, 1999.

[200] S. Tormanen et al. Phys. Lett. B, 454:8, 1999.

[201] D. Rudolph et al. Phys. Rev. Lett., 82:3763, 1999.

[202] T. Lauritsen et al. Phys. Rev. Lett., 89:282501, 2002.

[203] Ch. Finck et al. Phys. Lett. B, 467:15, 1999.

[204] E. Ideguchi et al. Phys. Rev. Lett., 87:222501, 2001.

[205] J. Robin et al. Phys. Rev. C, 78:034319, 2008.

[206] C.Svensson et al. Phys. Rev. Lett., 85:2693, 2000.

[207] B. Singh et al. Nucl. Dat. Sheets, 97:241, 2002.

[208] A. Lopez-Martens et al. Eur.Phys.J., A 20:49, 2004.

[209] K. Lagargren and B. Cederwall. Eur. Phys. J. A, 21:175, 2004.

[210] C. Andreoiu et al. Eur. Phys. J. A, 14:317, 2002.

[211] T. Bengtsson and I. Ragnarsson. Nucl. Phys. A, 1994:14, 436.

[212] R. L. Gill. Phys. Rev. C, 54:2276, 1996. 
[213] D. McNabb et al. Phys. Rev. C, 61:031304, 2000.

[214] A. Lopez-Martens et al. Phys. Rev. Lett, 77:1707, 1996.

[215] T. L. Khoo et al. Tunneling from super- to normal-deformed minima in nuclei. In S. Tomsevic, editor, Proceedings from the Institute for Nuclear Theory on Tunneling in Complex Systems, volume 5, page 229. World Scientific, 1998.

[216] H. Jackson. Phys. Rev. Lett., 17:656, 1966.

[217] C. Porter and R. Thomas. Phys. Rev., 104:483, 1956.

[218] S.J. Krieger et al. Nucl. Phys. A, 542:43, 1992.

[219] S.M. Harris et al. Phys. Rev., 130:509, 1965.

[220] P.-H. Heenen et al. Phys. Rev. C, 57:1719, 1998.

[221] O. Bohigas et al. Phys. Rev. Lett., 88:092502, 2002.

[222] J. Garrett. Phys. Lett. B, 392:24, 1997.

[223] G. Mitchell et al. Phys. Rev. Lett., 61:1473, 1988.

[224] B. Herskind et al. Acta Physica Polonica B, 38(4):1421, 2007.

[225] A.C. Larsen et al. To be published. 